\title{
Vivian EIDash
}

Influência de diferentes combinações de velocidade e inclinação durante a corrida na variabilidade da frequência cardíaca

\section{Influence of different combinations of speed} and slope during running on heart rate variability

São Paulo 
Vivian EIDash

\section{Influência de diferentes combinações de}

velocidade e inclinação durante a corrida na variabilidade da frequência cardíaca

Influence of different combinations of speed and slope during running on heart rate variability

Dissertação apresentada ao Instituto de Biociências da Universidade de São Paulo, para a obtenção de Título de Mestre em Fisiologia Geral

Orientador: José Guilherme Chaui-Berlinck

São Paulo 
EIDash, Vivian

Influence of different combinations of speed and slope during running on heart rate variability

Número de páginas 112

Dissertação (Mestrado) - Instituto de Biociências da Universidade de São Paulo. Departamento de Fisiologia.

1. Heart rate control 2. High intensity exercise. I. Universidade de São Paulo. Instituto de Biociências. Departamento de Fisiologia.

\section{Comissão Julgadora:}




\section{ACKNOWLEDGMENTS}

I guess this will be the most read page in my dissertation, so I should probably make it special, right? Well, that ain't gonna happen, it will be simple as I am not very good at this. I would first like to thank my parents for supporting me, not hurrying me up and most of all for stimulating education since we (me and my sisters) were kids. Second, I thank ZéGui for being a great advisor and friend. I still mix up enthalpy and entropy sometimes, but l've learned a lot. Thanks for all the coffee, for challenging me, all the runs and for being "soft butt" (it is hard to make people believe that). I also would like to thank my sis Gui, who was literally (for good or bad) by my side every step on the way, from uterus until now. Thiago, thanks for all the beers and company. Paulo, thanks for introducing me to 3 of my favorite things: beer, Iron Maiden and running. You are always right. Fefex, thanks for teaching me $R$ and for pointing out how post graduation is devalued. Finally, to Zé and Riley, thanks for putting up (with infinite patience) with all the drama that is dealing with me, for listening to my complains (and you know that I complain a lot) and comforting me when I cried (I do that a lot too). 


\section{LIST OF ABBREVIATIONS}

a

ANS

ApEn

AV

a1ApEn

BPM

C

$\mathrm{C}_{\text {nu }}$

DF

En exp

HF

$\mathrm{HF}_{\mathrm{nu}}$

HR

$\mathrm{HR}_{\text {max }}$

HRV

LF

$\mathrm{LF}_{\mathrm{nu}}$

LF:HF

rms

RPM

RSA

RSS

SA

sdnn

V

VLF

$\mathrm{VO}_{2 \max }$

$\mathrm{VO}_{2 \text { peak }}$

Wint
Angle in degrees

Autonomic nervous system

Approximate entropy

Atrioventricular node

Area 1 of approximate entropy

Beats per minute

Power $\left(\mathrm{s}^{2} / \mathrm{Hz}\right)$ in frequency band, from 1.3 to $1.6 \mathrm{~Hz}$

Normalized C, C power divided by the total spectral power

Degrees of freedom

Net energy expenditure $\left(\mathrm{O}_{2}\right.$ consumption per unit of distance per mass)

Power $\left(\mathrm{s}^{2} / \mathrm{Hz}\right)$ in the high-frequency band, from 0.15 to $0.8 \mathrm{~Hz}$

Normalized HF, HF power divided by the total spectral power.

Heart rate

Maximum heart rate

Heart rate variability

Power $\left(\mathrm{s}^{2} / \mathrm{Hz}\right)$ in the low-frequency band, from 0.04 to $0.15 \mathrm{~Hz}$

Normalized LF, LF power divided by the total spectral power

LF power divided by HF power, also referred to as "ratio"

Square root of the mean squared differences of successive normal RR

Rotations per minute

Respiratory sinus arrhythmia

Residual sum of squares

Sinus atrial node

Square root of the variance of successive normal RR intervals

Reference speed used for the experimental protocol

Power in the very low-frequency band, below $0.04 \mathrm{~Hz}$

Maximum oxygen consumption

Peak oxygen consumption

Internal work according to Cavagna \& Keneko (1977) 
Wext External work according to Cavagna \& Keneko (1977)

Wtot Total work done to move the body during locomotion

$Y_{\text {rel }} \quad$ Values of the HRV estimator $Y$ in a given condition divided by those obtained when running at $0 \%$ slope $V$ speed 


\section{TABLE OF CONTENTS}

1 INTRODUCTION 9

1.1 IS STEEPER SIMILAR TO FASTER? 9

1.1.1 Increments in speed or slope represent different stimuli to the musculoskeletal system 9

1.1.2 Increments in both speed and slope lead to increased metabolic $\begin{array}{ll}\text { demand } & 10\end{array}$

1.1.3 Can different combinations of speed and slope result in the same $\begin{array}{ll}\text { heart rate? } & 14\end{array}$

1.2 HEART RATE VARIABILITY 18

1.2.1 HRV response during exercise $\quad 19$

1.2.2 Effect of ventilatory frequency on HRV during exercise 24

1.2.3 Effect of cadence on HRV during exercise 25

1.2.4 Interactions between cadence, ventilatory frequency and heart $\begin{array}{ll}\text { rate } & 27\end{array}$

1.2.5 The problem of frequency-overlapping: concluding remarks $\quad 28$

2 OBJECTIVES AND HYPOTHESIS $\quad 30$

2.1 OBJECTIVES 30

2.1.1 General objectives $\quad 30$

$\begin{array}{ll}2.1 .2 \text { Specific objectives } & 30\end{array}$

2.2 HYPOTHESIS 31

3 MATERIALS AND METHODS 32

3.1 SUBJECTS 32

3.2 DATA ACQUISITION 32

3.3 SPEED SELECTION 33

3.4 EXPERIMENTAL PROTOCOL 34

3.4.1 Experiment 1

3.4.2 Experiment $2 \quad 34$

3.5 DATA PROCESSING 35

3.6 STATISTICAL ANALYSIS 36 
4 RESULTS AND DISCUSSION

4.1 QUESTION $1 \quad 40$

4.1.1 What is the equivalence of speed and slope regarding HR? 42

4.1.2 Does HR changes similarly along different running conditions? 43

4.1.3 In conclusion... 49

4.2 QUESTION $2 \quad 49$

4.2.1 Time domain estimators $\quad 49$

4.2.1.1 The standard deviation of RR intervals (sdnn) 49

4.2.1.2 Root mean square of successive differences of $R R$ intervals (rms) 52

4.2.1.3 Area 1 of Approximate Entropy (a1ApEn) 55

4.2.2 Frequency domain estimators $\quad 61$

4.2.2.1 LF band $(0.04-0.15 \mathrm{~Hz}) \quad 61$

4.2.2.2 HF band $(0.15-0.8 \mathrm{~Hz}) \quad 64$

$\begin{array}{ll}\text { 4.2.2.3 LF:HF ratio } & 67\end{array}$

4.2.2.4 Considerations regarding absolute power 70

4.2.2.5 Considerations regarding normalized power and LF:HF ratio 72

4.2.2.6 C band $(1.3-1.6 \mathrm{~Hz}) \quad 74$

$\begin{array}{ll}4.2 .3 \text { In conclusion } & 77\end{array}$

$\begin{array}{ll}\text { 4.3 QUESTION } 3 & 79\end{array}$

4.3.1 Time domain estimators $\quad 80$

4.3.1.1 Heart Rate $\quad 80$

4.3.1.2 The standard deviation of RR intervals (sdnn) 83

4.3.1.3 Root mean square of successive differences of $R R$ intervals (rms) 86

4.3.1.4 Area 1 of Approximate Entropy (a1ApEn) 89

$\begin{array}{ll}\text { 4.3.2 Frequency domain estimators } & \mathbf{9 0}\end{array}$

4.3.2.1 LF band $(0.04-0.15 \mathrm{~Hz}) \quad 90$

4.3.2.2 HF band $(0.15-0.8 \mathrm{~Hz}) \quad 93$

4.3.2.3 LF:HF ratio 96

4.3.2.4 Considerations regarding normalized power and LF:HF ratio 98

$\begin{array}{ll}\text { 4.3.2.5 } \mathrm{C} \text { band }(1.3-1.6 \mathrm{~Hz}) & 100\end{array}$ 
4.3.3 In conclusion

103

5 CONCLUSION

105

RESUMO

106

ABSTRACT

107

REFERENCES

108 


\section{INTRODUCTION}

\subsection{IS STEEPER SIMILAR TO FASTER?}

Most runners continuously face changes in speed and slope while exercising, whether during races, intrinsic to the surface where they usually train, or as a specific characterization of a given training session. The Saint Silvester Road Race, for example, one of the most popular races in Brazil, presents through its approximately $15 \mathrm{~km}$ route, an elevation gain of $\sim 190$ meters (HANADA, 2017). In the Boston Marathon, elevation gain is around 239 meters, while elevation loss is 373 meters (MACLIN, n. d.). It has been reported (VERNILLO et al., 2017) that hilly races are not as fast as those without changes in altimetry, even if net altitude change is close to zero. Since changes in slope and speed are present on the daily life of most runners, understanding their impact on physiology and running pattern is of great importance.

\subsubsection{Increments in speed or slope represent different stimuli to the musculoskeletal system}

In some aspects, mostly associated with the musculoskeletal system, changes in speed or slope represent very different stimuli to the runner. The pattern of muscle activation, for example, changes as slope increases. As shown by SLONIGER et al. (1997), when comparing a horizontal and $10 \%$ incline run, there is an increase in total muscle volume activated in the lower extremity. Such increase results from higher activation of some muscles (14\% in soleus and $23 \%$ in vastus group), and reduction of others (29\% on rectus femoris, $18 \%$ on gracilis and $17 \%$ on semitendinosus). EMG data additionally supports greater activity in vastus lateralis and gluteus maximus during uphill running (VERNILLO et al., 2017).

Biomechanics of running uphill is not the same as that presented on a horizontal surface. Running on a positive slope is associated with a tendency to $\mathrm{mid} /$ forefoot strike, higher stride frequency when compared to level running and 
decreased stride length, resulting in an increased internal mechanical work, shorter aerial phase duration and longer time spent in stance (VERNILLO et al., 2017).

PADULO et al. (2013) investigated the biomechanics of 18 highly trained male runners running on a treadmill at the velocity associated with $70 \%$ maximum oxygen consumption $\left(\mathrm{VO}_{2 \text { max }}\right)$, on $0 \%, 2 \%$, and $7 \%$ incline. They reported a $2 \%$ and $4 \%$ increase in the stride frequency when comparing level running on the lowest and highest slopes tested, respectively. Stride length decreased at approximately the same magnitude. At higher slopes, they reported a decrease in the aerial phase and a concomitant increase in ground contact time.

When speed is increased instead of the slope, a different pattern of locomotion is observed. In submaximal running (up to $\sim 90 \%$ maximum velocity), increments in speed are mainly met by increased stride length, resulting in more time spent on swing phase (NOVACHECK, 1998; DUGAN \& BHAT, 2005; STÖGGL \& WUNSCH, 2016). In fact, MANN \& HAGY (1980) reported a reduction in ground contact phase duration from $31 \%$ to $22 \%$ in running and sprinting, respectively. On the other hand, increased stride frequency will be more significant in attaining higher speeds only when velocity approaches maximum. Under such circumstances, total gait time is reduced.

\subsubsection{Increments in both speed and slope lead to increased metabolic demand}

If on the one hand running faster or on a steeper surface causes notably different responses in the skeletal system, those stimuli are both associated with increased metabolic demand.

Consider displacing a mass $M$ on an inclined surface without friction, at an angle a and a given speed $v$ (Fig. 1). The total power, $\mathrm{W}$, can be expressed as:

$$
W=M \cdot v \cdot \cos (a)+M \cdot g \cdot v \cdot \operatorname{sen}(a)
$$

The first right-hand term of the equation, that is, $M \cdot v \cdot \cos (a)$, refers to the power performed on the horizontal axis. The remainder is associated with the displacement observed in the vertical axis, that is, changes in potential energy. If the 
surface is horizontal, $\cos (a)=1$ and $\operatorname{sen}(a)=0$, therefore, under this particular scenario, power will be dependent only on mass and velocity. Equation (1) may be reorganized to:

$$
W=M \cdot v \cdot(\cos (a)+g \cdot \operatorname{sen}(a))
$$

In that way, it is clear that changes in speed are linearly and directly related to power.

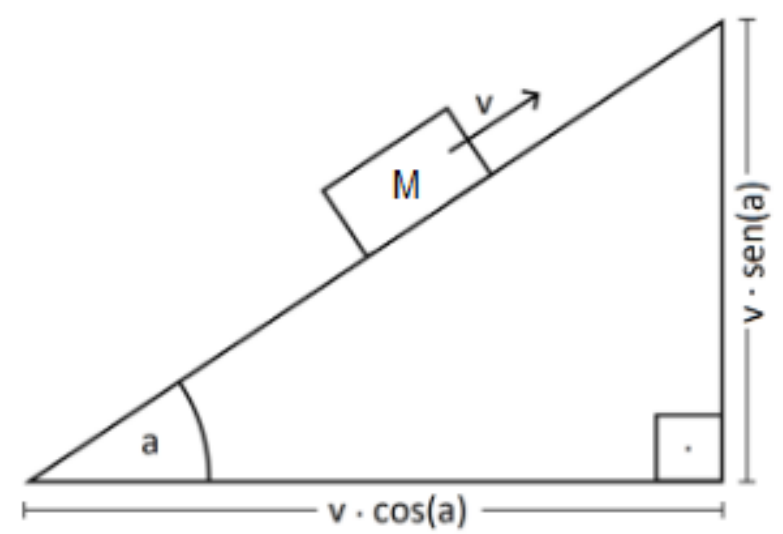

Figure 1 - Representation of an object of mass $M$ being moved at velocity $v$ on an inclined surface with an angle $a$.

The above-mentioned equation is obviously not intended to represent human locomotion, but to present what could have been expected considering the simplest possible scenario of mass displacement.

CAVAGNA \& KANEKO (1977) estimated the total work done to move the body during locomotion per mass per unit of distance (Wtot) for a variety of speeds, ranging from 3 to $33 \mathrm{~km} / \mathrm{h}$. Wtot was calculated as being the sum of the external work, performed to accelerate the body center of mass (Wext) and the internal work, associated with limb segments displacement relative to the trunk (Wint).

During running, Wint was found to be is proportional to approximately the square of the velocity and was smaller than Wext up to about $20 \mathrm{~km} / \mathrm{h}$. Moreover, Wtot was found to increase with speed (Fig. 2). 
WORK AND EFFICIENCY IN HUMAN LOCOMOTION

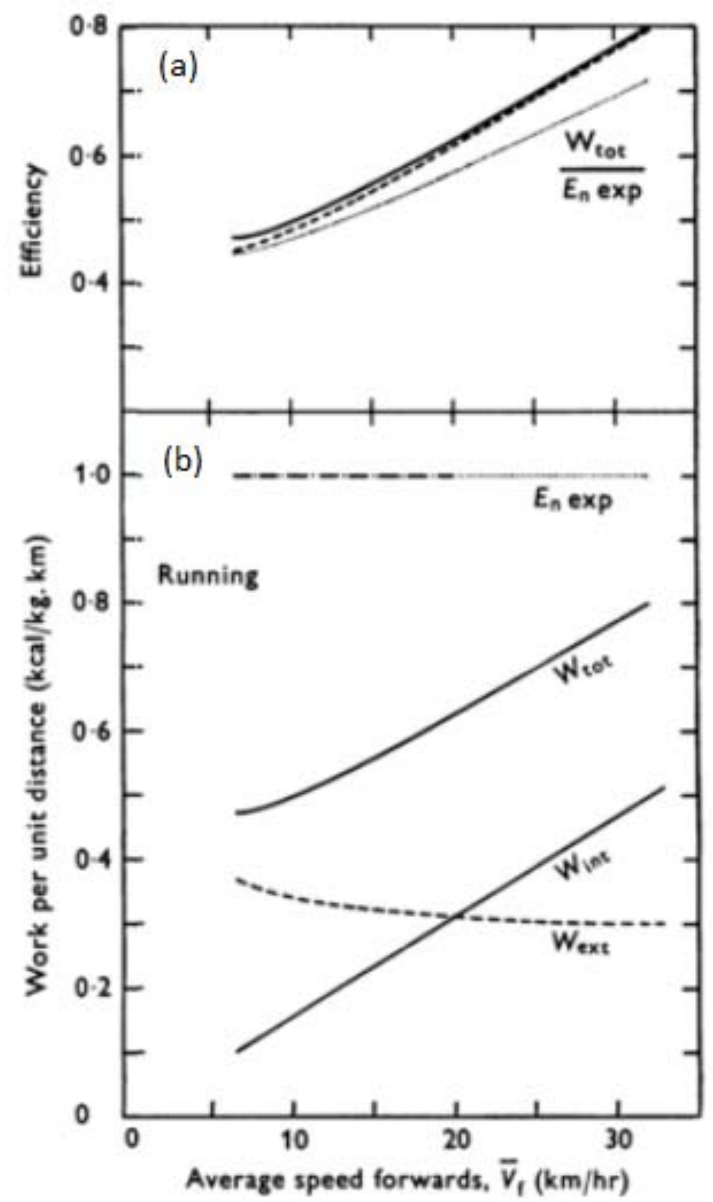

Figure 2 - Mechanical efficiency (a) and mechanical work per mass per unit distance (b) as functions of speed. En exp is net energy expenditure, Wtot is total work performed, Wint internal work and Wext is external work. Note that efficiency increases with speed due to an increment in Wtot. As velocity increases, so does Wint, a feature not observed in Wext. At speeds higher than $\sim 20 \mathrm{~km} / \mathrm{h}$, Wint is greater than Wext. Adapted from "Mechanical work and efficiency in level walking and running", by CAVAGNA, G. \& KANEKO, M., 1977, Journal of Physiology, 268 (3), p. 475.

In order to estimate running efficiency, the authors divided Wtot per En exp. En exp is net energy expenditure per mass per unit of distance, total minus standing, and such value is constant. The coefficient of the regression line between oxygen consumption rate and speed is roughly 1 , the same predicted by equation 2 .

Cost of running, measured as both oxygen uptake $(\mathrm{ml} /(\mathrm{kg} \cdot \mathrm{m}))$ and caloric $\operatorname{cost}(\mathrm{J} /(\mathrm{kg} \cdot \mathrm{m}))$, increases linearly with increasing slope, mainly due to the additional 
energy required to elevate the body center of mass. Such a net increase in the body's potential energy is met by a higher mechanical work performed by leg muscles, therefore, there is a greater muscle activity at all joints, mainly the hip. A secondary factor that might contribute to the increased metabolic demand during uphill running is a reported reduction in the maximum possible elastic energy stored and released by tendons under such circumstances (VERNILLO et al., 2017).

MINETTI et al. (2002) measured the cost of locomotion of 10 subjects on a treadmill in an ample range of slopes and speeds (here, only the results regarding uphill running are presented). As expected, oxygen consumption rate increased linearly with speed. Energy expenditure per meter was constant, despite running speed, but changed according to the slope. At level running, cost of locomotion was estimated as $3.4($ s.d. $=0.24) \mathrm{J} /(\mathrm{kg} \cdot \mathrm{m})$, and reached $18.93(\mathrm{~s} . \mathrm{d}$. $=1.74) \mathrm{J} /(\mathrm{kg} \cdot \mathrm{m})$ at $45 \%$ incline.

Interestingly, at slopes above $15 \%$ incline, the cost of running was linearly proportional to the slope and corresponded to a mechanical efficiency of $0.22-0.24$ (kinetic energy was regarded as negligible). Such data indicates that, as previously hypothesized (MARGARIA, 1938 and MARGARIA et al., 1963 apud MINETTI, 2002), at sufficiently high slopes, locomotion efficiency corresponds to that of concentric muscle contraction.

In the aforementioned paper, the authors employed a 5th order polynomial equation to describe the relationship between the cost of running and a given gradient. Such an equation was estimated including both positive and negative slopes, and data collected from other studies as well. Using data from their reported experiment for positive slopes only, we recalculated cost of running $(\mathrm{Cr})$ and found that it could be described as linearly proportional to incline in percentage $(\theta)$, according to equation 3 below $\left(r^{2}=0.98\right.$, $p$-value $\left.<0.001\right)$ :

$C r=2.6+34.8 \theta$

Such phenomena result from only positive work being performed when the slope is higher than 0.15 . Under such circumstances, the descending phase of the bouncing ball movement pattern associated with running loses importance, therefore, 
there is no recovery of elastic energy. As a consequence, all the work done to lift the body is generated by muscle contraction (MINETTI et al., 2002).

When comparing running on $0 \%$, to $2 \%$ and $7 \%$ incline, PADULO et al. (2013) observed a $14 \%$ and $53 \%$ increase in metabolic cost. Such pattern resulted from increments in both oxygen consumption and accumulated blood lactate (i.e., anaerobic metabolism). Heart rate increased $5 \%$ when comparing level running to $2 \%$ incline (from 148 BPM to 155 BPM, beats per minute) and 15\% under 7\% incline (reaching 170 BPM).

\subsubsection{Can different combinations of speed and slope result in the same heart rate?}

Since changes in both speed and slope have a pronounced impact on energy expenditure, it comes as no surprise that a number of different combinations between these two variables can result in the same metabolic rate. Under a primarily oxidative metabolism and steady-state conditions, the heart rate is a good linear indicator of metabolic rate (MCARDLE et al., 2006).

Despite not being their main objective, the study of JONES \& DOUST(1996) reports some interesting experimental data on such matter. Nine trained male runners engaged in the study. The experimental protocol consisted of a series of 6 -minute runs on a treadmill at 6 velocities $(2.92,3.33,3.75,4.17,4.58$ and $5.0 \mathrm{~m} / \mathrm{s})$. Each run was followed by a recovery period and the procedure was repeated on different days for $0 \%, 1 \%, 2 \%$, and $3 \%$ incline. The results obtained are reproduced in Table 1.

In Table 1 we can see, for example, that running at $3.75 \mathrm{~m} / \mathrm{s}$ at $3 \%$ grade leads to the same heart rate as $4.17 \mathrm{~m} / \mathrm{s}$ at $1 \%$ grade (153 BPM). Also, a $3.33 \mathrm{~m} / \mathrm{s}$ speed at $0 \%$ grade (124 BPM) appears to be similar to $2.92 \mathrm{~m} / \mathrm{s}$ at $2 \%$ grade (126 BPM). 
Table 1 - Heart rate (BPM) under each run presented as mean (s.d).

\begin{tabular}{lcccc}
\hline \multirow{2}{*}{$\begin{array}{l}\text { Running } \\
\text { velocity }(\mathrm{m} / \mathrm{s})\end{array}$} & \multicolumn{4}{c}{ Slope } \\
\cline { 2 - 5 } & $0 \%$ & $1 \%$ & $2 \%$ & $3 \%$ \\
\hline 2.92 & $116(7)^{\mathrm{c}, \mathrm{d}}$ & $119(8)^{\mathrm{c}, \mathrm{d}}$ & $126(7)^{\mathrm{a}, \mathrm{b}}$ & $132(6)^{\mathrm{a}, \mathrm{b}}$ \\
3.33 & $124(6)^{\mathrm{c}, \mathrm{d}}$ & $128(7)^{\mathrm{d}}$ & $134(7)^{\mathrm{a}}$ & $141(6)^{\mathrm{a}, \mathrm{b}}$ \\
3.75 & $137(6)^{\mathrm{c,d}}$ & $140(6)^{\mathrm{d}}$ & $143(6)$ & $153(7)^{\mathrm{a}, \mathrm{b}}$ \\
4.17 & $149(6)^{\mathrm{c}, \mathrm{d}}$ & $153(7)^{\mathrm{d}}$ & $155(7)$ & $164(7)^{\mathrm{a}, \mathrm{b}}$ \\
4.58 & $160(7)^{\mathrm{c,d}}$ & $164(7)^{\mathrm{d}}$ & $166(9)$ & $174(9)^{\mathrm{a}, \mathrm{b}}$ \\
5.00 & $171(9)^{\mathrm{c}, \mathrm{d}}$ & $173(8)^{\mathrm{d}}$ & $175(9)$ & $186(11)^{\mathrm{a}, \mathrm{b}}$
\end{tabular}

Significantly different ( $p$-value < 0.05 ) from $0 \%(a), 1 \%(b), 2 \%(c)$ and $3 \%(d)$ incline. Note. Adapted from "A $1 \%$ treadmill grade most accurately reflects the energetic cost of outdoor running", by Jones, A. \& Doust, J., 1996, Journal of Sports Science, 14 (4) , p. 324.

An interesting way to look at such data is to construct heart rate $(H R)$ as a surface function from speed and incline, and, then, analyze the gradient of the surface. Such analysis conveys information on the magnitude and orientation of HR changes. The gradient according to equation 4 :

$$
\nabla(H R)=(\partial H R / \partial u) \hat{\mathrm{u}}+(\partial H R / \partial \theta) \hat{\imath}
$$

Where $u$ is velocity, $u$ the axis associated with velocity, $\theta$ is the slope and $\hat{i}$ the axis associated with slope ( $\hat{u}$ and $\hat{i}$ are orthogonal).

In Fig. 3 we represent the magnitude $(M)$ of HR change as a function of speed and slope, calculated as:

$$
M=\sqrt{(\partial H R / \partial u)^{2}+(\partial H R / \partial \theta)^{2}}
$$

In order to better compare the magnitude changes in the data obtained from JONES \& DOUST(1996) to that collected by us (see "Results and Discussion", 
Question 1), HR was made relative to the run performed at $4.58 \mathrm{~m} / \mathrm{s}, 0 \%$ incline. Such condition is similar to that we used to select our reference speed, $V$, that is, running at a horizontal surface at approximately $84 \%$ of estimated maximum HR. As subjects studied by JONES \& DOUST(1996) had on average 25 years, target HR was 160 BPM (see "Materials and Methods" section for details), exactly the value obtained when running at $4.58 \mathrm{~m} / \mathrm{s}, 0 \%$ incline.

If HR changed by the same amount irrespectively to the speed and to the slope, we would find that the magnitude does not change through the range of conditions studied. Observing Fig. 3, calculated using data reported by JONES \& DOUST(1996), a clear pattern cannot be identified. The magnitude of changes is within a relatively narrow range. This shows that HR changes approximately by the same amount everywhere in the space of speed and slope.

The angle $\left(G_{i}\right)$ is a quantification of how factors analyzed (slope or speed) affect the observed changes in the variable of interest (HR). An angle of 45 degrees means that speed and slope have the same impact on HR. In other words, doubling speed increases HR by the same amount as doubling slope. As the angle approaches 90 degrees, the relative importance of speed increases, while an angle closer to zero indicates a bigger impact of the slope. A positive value of the angle means both speed and slope changes HR in the same direction, while the opposite stands for a negative angle. The angle is calculated according to the equation:

$G_{i}=(\partial H R / \partial u) /(\partial H R / \partial \theta)$

In Fig. 4 we show the angle of changes in HR calculated from data obtained by JONES \& DOUST(1996). We can observe that as the slope increases, it's relative impact on HR also increases.

Such analyses, along with the aforementioned studies bring some light into the effects of speed and slope on metabolic demand and on the locomotor system. Therefore we can better understand how running at different speeds and on a graded surface presents its own challenges, and leads to distinct responses regarding not only the locomotor but also the cardiovascular system. 


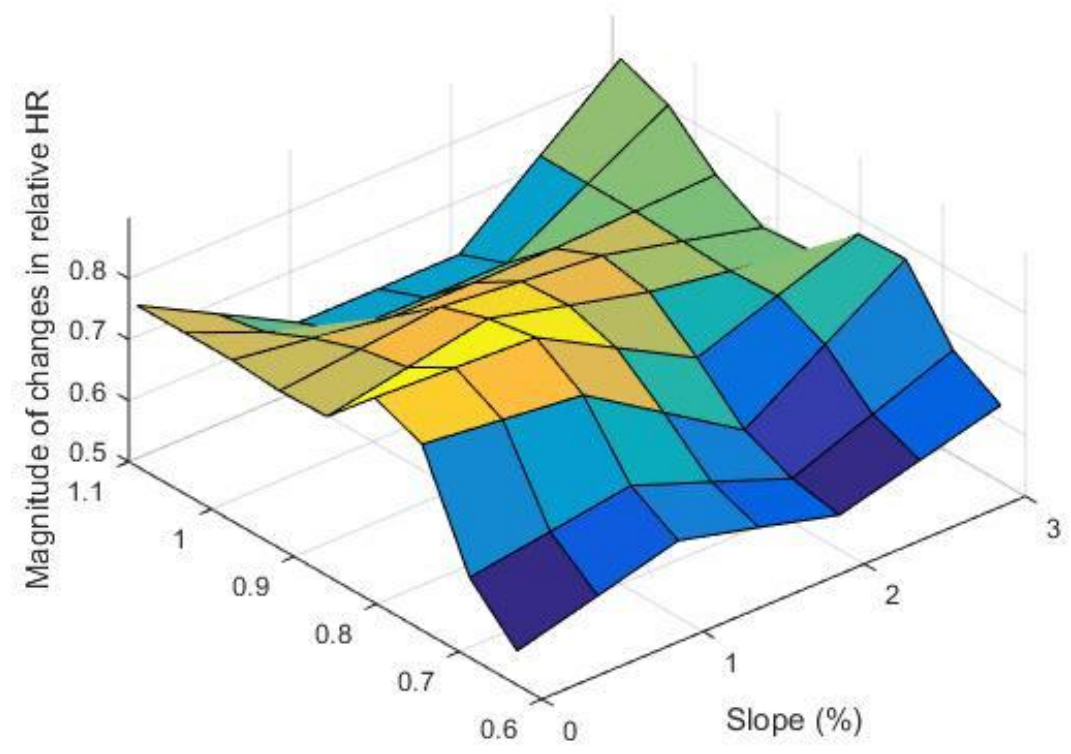

Speed (fraction of V)

Figure 3 - The magnitude of the surface gradient of $H R$ as a function of speed and slope. Magnitudes were calculated from interpolated surface data from JONES \& DOUST(1996).

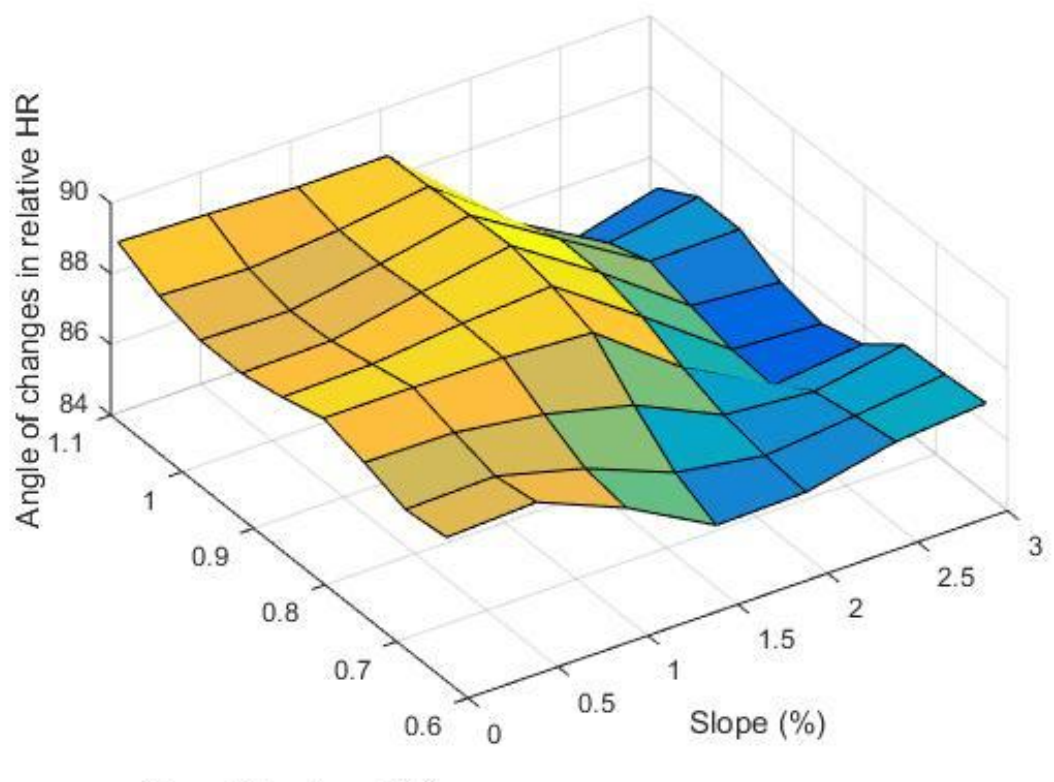

Speed (fraction of V)

Figure 4 - The angle of the surface gradient of $H R$ as a function of speed and slope. Angles were calculated from the interpolated surface data from JONES \& DOUST(1996). 


\subsection{HEART RATE VARIABILITY}

Heart rate is modulated by the autonomic nervous system (ANS), particularly by a region called cardioregulatory center located in the medulla oblongata. Both the sympathetic and parasympathetic branches innervate the heart. The parasympathetic nerves are located mainly in the atria, sinus atrial (SA) and atrioventricular (AV) nodes. Vagal stimulation reduces $\mathrm{HR}$, conduction velocity and contraction force. The sympathetic nerve fibers innervate both $S A$ and $A V$ nodes as well as atrial and ventricular walls. Sympathetic stimulation leads to an increase in HR, conduction velocity and contraction force. Autonomic control responds to a diversity of afferences, including baroreceptor, chemoreceptor, humoral, muscular and environmental inputs (DONG, 2016).

Even under steady-state conditions, there are instantaneous changes in heart rate, i.e. the time interval between successive heartbeats is not constant. The pattern associated with heart rate variability (HRV) is typically nonlinear, nonstationary, multiscale variable and temporarily asymmetric, making heart rate a remarkably complex signal. Such complexity is thought to result from heart rate control mechanisms response to internal and external stimuli. Periodic or random signals indicate malfunctioning of control mechanisms and/or of the controlled system (the heart itself), as in pathological conditions (GOLDBERGER et al., 2002). Therefore, how HRV behaves under different conditions can shed light into cardiac control and the operation of the cardiovascular system (GUIDELINES, 1996; PERINI \& VEICSTEINAS, 2003; DONG, 2016).

HRV can be analyzed, among other methods, by decomposing the original signal into its component waves. Typically, the resultant spectrum is divided into at least 3 frequency bands: (1) very low-frequency frequency - VLF, below $0.04 \mathrm{~Hz}$; (2) low frequency band - LF, from 0.04 to $0.15 \mathrm{~Hz}$ and (3) high frequency band - HF, from 0.15 to $0.4 \mathrm{~Hz}$ or above (GUIDELINES, 1996; PERINI \& VEICSTEINAS, 2003)

At rest, heart rate fluctuations in the $\mathrm{HF}$ band frequencies, i.e., above $0.15 \mathrm{~Hz}$ up to $0.4 \mathrm{~Hz}$, are taken to be influenced by the parasympathetic branch of the ANS, mainly associated with respiratory sinus arrhythmia (RSA). A peak at the respiratory rate is frequently observed. In contrast, fluctuations in the LF band are considered to 
be resultant from both the parasympathetic and sympathetic branches, related to baroreceptor activity involved in arterial pressure control, and the renin-angiotensin system. VLF interpretation is more controversial, but some authors associated this frequency band with peripheral vasomotor regulation (GUIDELINES, 1996; PERINI \& VEICSTEINAS, 2003).

\subsubsection{HRV response during exercise}

During exercise, however, interpretation of HRV is still controversial (BAILÓN et al., 2013). Evidence suggests that the spectral component bands fail to reflect ANS branches activity (MICHAEL et al., 2017), and analysis of HRV through such method should be seen as readjustments of the control mechanism (PERINI \& VEICSTEINAS, 2003). Amongst the complicating factors in analyzing HRV spectrum during exercise are non-stationarity of signal (i.e. statistical properties of the system change with time), the introduction of cardiolocomotor coupling and overlapping between LF or HF bands with ventilatory and locomotor frequencies.

Despite its challenges, understanding how HRV behaves during physical activity may bring light into exercise physiology as well as potentially contribute to athletic training (ALIKHANI et al., 2017). Some studies indicate that monitoring HRV (both during and after the exercise, and while performing some maneuvers) might represent a useful marker of training adjustment and a piece of relevant information to setting training loads (DONG, 2016).

Exercise intensity seems to be the most important factor in HRV behavior (MICHAEL et al., 2017). At the onset of exercise, an increase in HR of approximately 30 beats per minute is achieved through vagal withdrawn, while further increments are possible due to increased sympathetic tone (PERINI \& VEICSTEINAS, 2003). As HR increases, HRV decreases (BAILÓN et al., 2013; MICHAEL et al., 2017). As a result, the total power in the spectrum, as well as in each spectral component, is reduced (PERINI \& VEICSTEINAS, 2003). Most of the observed reduction in total power takes place during the first stages of graded exercise, at $\sim 25-30 \%$ of $\mathrm{VO}_{2 \max }$, when increments in HR are mainly due to vagal withdrawn (PERINI \& VEICSTEINAS, 
2003). Some studies reported a biphasic behavior of HRV during exercise, variability was reduced up to $\sim 60 \%$ of maximum intensity but increased at higher intensities (BLAIN et al., 2009).

Based on what is known regarding autonomic control of heart rate during physical activity (briefly, as intensity increases parasympathetic tone decreases and sympathetic tonus is augmented), when comparing exercise to rest, a reduction in HF power and increase in LF power would be expected when expressed as normalized units, resulting in a tendency to increase LF/HF ratio values.

In absolute values, both LF and HF power are reduced during physical activity, reflecting the observed decrease in total spectral power (BAILÓN et al., 2013; DONG, 2016). When power in each band is presented as normalized units, results often do not follow expectations (MICHAEL et al., 2017). PERINI \& VEICSTEINAS (2003) reviewed a series of experiments performed by their group and reported no changes in the percentage of LF power under low-intensity exercise. At intensities above $30 \% \mathrm{VO}_{2 \max }$, LF percent decreased linearly and nearly disappeared at higher intensities. During moderate intensity, LF peak has been shown to shift to lower frequencies, resulting in a decrement in power in such band, and increased power in VLF band. The HF power remained unchanged from rest up to approximately $70 \%$ $\mathrm{VO}_{2 \max }$, despite the decreased vagal tonus. A peak at HF frequencies could be observed from low to maximum exercise load. At maximum workload, HF peak accounted for most of the variability in HR (PERINI \& VEICSTEINAS, 2003).

SUMI et al. (2006) studied the HRV power spectrum in 10 female runners during a $3 \mathrm{~km}$ time trial and obtained similar results. Compared to rest, a significant reduction in total power and in the LF band was observed. The LF/HF ratio presented a biphasic behavior, increasing during the first $300 \mathrm{~m}$, and decreasing to nearly zero at the $900 \mathrm{~m}$ until the end of the exercise. A peak at HF band (considered from 0.15 to $1 \mathrm{~Hz}$ ) could be identified throughout the entire exercise and presented cross-correlation values with stride frequency ranging from 0.70 to 0.87 (the influence of stride frequency on HRV will be discussed later). The authors concluded "[HRV] to be a poor method to evaluate the cardiac autonomic nervous activity during high-intensity exercise when exceeding a heart rate of 175 BPM". 
Other studies also reported that normalized HF power was reduced under low-intensity exercise and increased with increasing workload, persisting during moderate (PERINI \& VEICSTEINAS, 2003; MICHAEL et al., 2017) and maximum (CASADEI et al., 1995; PERINI \& VEICSTEINAS, 2003; MICHAEL et al., 2017) intensity while LF power was unaltered or increased under low-medium intensity, but was reduced at higher intensities (PERINI \& VEICSTEINAS, 2003; BAILÓN et al., 2013; DONG, 2016), possibly as a consequence of vagal withdrawn (BAILÓN et al., 2013).

Aside from the power spectrum, other tools can be used to estimate HRV. Standard deviation (the square root of the variance, sdnn) is frequently calculated. As variance is analytically equivalent to the total power of spectral analysis, sdnn is influenced by all frequency components, and its values are reduced when comparing exercise to rest. The square root of the mean squared differences of successive normal RR intervals ( $\mathrm{rms}$ ) is also frequently calculated. Such estimator is particularly sensitive to high frequencies in HRV, therefore it presents a high correlation to the HF band power (GUIDELINES, 1996).

The use of non-linear tools could bring additional information in understanding HRV (GUIDELINES, 1996). One important class of estimators are entropies, which address the system's organization. A variety of information entropies have been developed since the work of Shannon. One of such entropies was devised by Pincus, the so-called Approximate Entropy (ApEn - PINCUS, 1991). Due to its very easy computation, ApEn became extensively employed to characterize the organization of time-series in a wide range of research areas. Also, it served as a basis for another set of ApEn-based entropies.

Briefly, ApEn is calculated as the number of equal events (\#) observed, with a certain tolerance $r$, when comparing a sub-vector $i$ with a window size $m$ along the time series (composed of $N$ values) to those observed when the window size is increased by one unit $(m+1)$. Precisely, ApEn can be obtained according to equations 7 - 9:

$$
C_{i}^{m}(\mathrm{r})=\frac{\#_{i}^{m}}{N-m+1}
$$


From this value, $\phi$ is computed:

$\Phi^{m}(r)=\frac{1}{N-m+1} \sum_{1}^{N-m+1} \ln \left(C_{i}^{m}\right)$

Finally, ApEn is obtained as:

$\operatorname{ApEn}(m, r, N)=\Phi^{m}(r)-\Phi^{m+1}(r)$

More details can be obtained on PINCUS (1991). As we can see, more predictable and less complex time series will be associated with lower ApEn values.

As pointed out in previous works (SANTOS et al., 2009; NATALI \& CHAUI-BERLINCK, 2016), ApEn presents an undesirable lack of objectivity since the choice of the parameters $r$ and $m$ are arbitrary and are decided by the researcher. This leads to some inconsistencies in the comparisons among diverse time-series. Such drawback motivated the development of a1ApEn (NATALI \& CHAUI-BERLINCK, 2016), a complexity estimator based on ApEn, computed as the area under the curve formed by ApEn values calculated as $m=1$ for all possible and relevant $r$ values (equation 10):

$a 1 A p E n=\int_{0}^{1} \operatorname{ApEn}\left(m=1, r^{*}, N\right) d r^{*}$

Where $r^{*}$ indicates a normalized tolerance vector. For further details, please refer to the aforementioned study.

Some studies have analyzed HRV through non-linear tools, particularly ApEn or derived methods. When estimating HRV through ApEn, higher values were observed in signals from healthy individuals, while lower ApEn was obtained in abnormal HR signal (ACHARYA et al., 2006).

TULPPO et al. (2001) analyzed responses of HRV to passive tilt test and low-intensity exercise using ApEn and detrended fluctuation analysis. Detrended fluctuation analysis consists of plotting the root mean square fluctuation measured in windows of different sizes from the original integrated detrended signal, against the size of the window on a log-log scale. The slope of such line is the scaling exponent and is a measure of fractal-like correlation properties in the HR signal (TULPPO et 
al., 2001). Increased fractal exponent was observed when comparing rest to both tilt test and low-intensity exercise, indicating stronger correlation properties in the HR signal. On the other hand, ApEn only increased under exercise condition. Such results indicate that ApEn was able to identify a different HR control generated by those experimental conditions that were not perceived by other non-linear methods.

The study of NATALI (2015) indicates that HRV, estimated through a1ApEn, is influenced both by exercise duration and intensity. The author analyzed HRV from subjects under 3 running protocols: constant, increasing and decreasing speeds. A positive relationship was observed between a1ApEn and the experiment duration under the increasing speed protocol. Negative and positive non-significant correlations were present in the decreasing and constant speed protocol, respectively. The author interpreted that both factors analyzed, exercise duration and velocity, contributed to the observed complexity in HR signal, and their effects were highlighted by the incremental protocol. Additionally, when HRV was analyzed as a function of $\mathrm{HR}$, the correlation between a1ApEn and HR was predominantly positive and statistically significant for all protocols, indicating that complexity varies with HR.

A complicating factor when analysing HRV data during exercise in general, and specifically at high intensity running, is that, as previously mentioned, other components associated with the physical activity itself, such as cadence and ventilatory frequency, influencethe HRV spectrum, and should be taken into consideration when interpreting data (ALIKHANI et al., 2017). For example, results from graded cycling test in different fitness groups indicate that, under low-intensity exercise, the component associated with RSA is the most important contributor to total power (BLAIN et al., 2009; BAILÓN et al., 2013). As for maximal graded exercise, around $40 \%$ of HRV was associated with cadence, and $60 \%$ with RSA (BLAIN et al., 2009). 


\subsubsection{Effect of ventilatory frequency on HRV during exercise}

Part of the observed HRV is associated with ventilation. Under rest, ventilatory frequency falls within the HF band defined as ranging from 0.15 to $0.4 \mathrm{~Hz}$ (GUIDELINES, 1996). However, depending upon activity intensity, the ventilatory frequency may exceed the $0.4 \mathrm{~Hz}$ that traditionally characterizes the upper limit of the HF band (that is, more than 24 cycles per minute). For that reason, some authors choose to extend the upper limit of $\mathrm{HF}$ to $1 \mathrm{~Hz}$, encompassing the full range of ventilatory frequencies (PERINI \& VEICSTEINAS, 2003; BAILÓN et al., 2013; ALIKHANI et al., 2017).

Studies have shown that a peak at respiratory rate can be observed in the power spectrum of HRV (PERINI \& VEICSTEINAS, 2003; SUMI et al, 2006). Moreover, HF central frequency has been reported to increase almost linearly with exercise intensity, indicating the persistence of ventilatory modulation on HRV under all exercise intensities. The observed increment in HF central frequency as a function of exercise intensity seems to be affected by other factors besides metabolic demand. It has been hypothesized the amplitude of HF modulation to be related to absolute $\mathrm{VO}_{2}$. This possibility is supported by a similar increment in HF percent in both male and female elderly exercising at the same range of metabolic demand, nevertheless, males presented higher values of $\mathrm{HF}$ and $\mathrm{VO}_{2 \text { peak }}$ (PERINI \& VEICSTEINAS, 2003).

While the decrease in HF power observed at low-moderate exercises corroborates the interpretation of HF as a marker of parasympathetic control of HR under physical activity, the increase in the power of the normalized HF band reported at exercises of higher intensities indicates that HRV modulation under such conditions does not reflect only the cardiac vagal control.

BAILÓN et al. (2013) hypothesized the high values of normalized HF observed under medium-high intensity exercise to reflect mechanical stretching of the sinus node associated with exercise-induced hyperventilation above the anaerobic threshold. During inspiration, the reduction of intrathoracic pressure would lead to a greater venous return and, consequently, a greater atrial filling. This, in turn, leads to stretching of the SA node, raising HR. Therefore, the modulatory effect of ventilatory 
frequency on HRV during exercise would be mainly associated with a mechanical, rather than a neural, effect.

This hypothesis is also supported by the persistence of a ventilatory rhythm in HR detected in heart-transplanted patients during exercise, despite significantly reduced HF power under rest when compared to healthy subjects (PERINI \& VEICSTEINAS, 2003).

\subsubsection{Effect of cadence on HRV during exercise}

The existence and quantitative relevance of a cadence component in modulating HR have been previously demonstrated, either through HRV analysis or more usually as synchronization between step cycle and occurrence of the $R$ wave (KIRBY et al., 1989; BLAIN et al., 2009; ALIKHANI et al., 2017).

Some studies found cardiolomotor coupling to be an intermittent phenomenon only existent when HR and cadence were of similar values. KIRBY et al. (1989), for example, analyzed cardiac and locomotor synchronization when running on a treadmill at velocities ranging from 4 to $17 \mathrm{~km} / \mathrm{h}(\mathrm{N}=25)$. They reported cardiolocomotor coupling to be present in 18 volunteers, but not at all speeds and usually at the moderate-high intensity. NIIZEKI et al. (1993) similarly reported cardiolocomotor coupling in most volunteers in at least one analyzed speed, independently of workload. However, even under constant velocity, synchronization periods interspersed with non-synchronization were observed on a minute by minute basis.

Such results raise the possibility that cardiolocomotor coupling could be merely the result of the intersection between locomotor and heart rates, observed by chance. The study published by BLAIN et al. (2009) goes against this hypothesis, reporting cardiolocomotor coupling under a wide range of heart rates and different cadences. The experimental protocol adopted in the aforementioned study consisted of a maximal graded cycling test performed at a fixed cadence $(70,80$ or 90 rotations per minute, RPM - 1.17, 1.33 and $1.50 \mathrm{~Hz}$, respectively). A total of 15 individuals, with different fitness levels, engaged in the study and were assigned to a given 
cadence group according to their preferred pedaling frequency. No increase in the frequency band associated with cadence was observed from 30 to $50 \%$ of maximum power output in the 70 and 80 RPM groups when compared to its minimum values. As for the 90 RPM group, spectral aliasing overlapped with ventilatory frequency, making it difficult to identify what fraction of the observed power was due to RSA or cadence component. At higher intensities, from 60 to $100 \%$ of maximum workload, the power associated with cadence frequency increased $\sim 3$ fold in all groups, accounting from 39 to $49 \%$ total HRV under maximum intensity exercise. Results presented a similar pattern when expressed as the percentage of total power. The authors concluded that cycling cadence is a component of HRV, and cardiolocomotor coupling is accentuated by increased workloads.

Unlike BLAIN et al. (2009), BAILÓN et al. (2013) analyzed HRV of 23 subjects under an incremental running protocol on the treadmill and failed to observe an increased power in the band associated with cadence at higher workloads. Regardless of exercise intensity, stride frequency accounted for $22 \%$ total power. As pointed out by the authors, a possible explanation lies in the fact that they considered locomotor frequency power as being the sum of cadence frequency power and its aliases.

ALIKHANI et al. (2017) analyzed the influence of the component on HRV as well as movements along the horizontal and sagittal axis during incremental running protocol on a treadmill. They concluded that cadence frequency is the main component, but movements along the horizontal axis, possibly originating from arm swing or torso movement, also contribute with significant power to HRV spectrogram.

One possible explanation to cardiolocomotor synchronization is of a mechanical origin, in which rhythmic muscle contraction modulates venous return (BLAIN et al., 2009). Under dynamic muscle contractions, venous return presents a pulsatile pattern, increasing during contraction and decreasing in the relaxation phase (FOLKOW et al., 1970). Such a hypothesis is further supported by the proportional relationship observed between power in the cadence component and workload (BLAIN et al., 2009).

FISHER (2014) reviewed some mechanisms behind the influence of skeletal muscle afferents on the control of HR. 
Activation of mechanoreceptors on skeletal muscles leads to an increase in HR through suppression of the parasympathetic activity. This mechanism is particularly important at the onset of exercise and at low-intensity physical activity. Its relevance is such that increments in HR have been observed during electrically stimulated muscle contractions even when the central command, both cardiovascular and somatomotor centers, are bypassed.

Metaboreceptors in muscular tissue evoke an increase in HR as well, but via activation of the sympathetic branch. They also play a role in the sympathetic regulation of the skeletal muscle vasculature during exercise (FISHER, 2014).

To further elucidate this mechanism, FISHER et al. (2013, apud FISHER, 2014) studied the effects of moderate intensity cycling with partial flow restriction on cardiovascular responses under control condition, adrenergic and vagal blockade. They reported that the increase in HR was reduced by $50 \%$ with the adrenergic, but not the parasympathetic blockade, reiterating the role of the sympathetic system in this mechanism. Authors also point out that since the increase in HR was not completely abolished upon sympathetic blockade, other factors might play a role, such as central command and/or muscle mechanoreceptors. Moreover, it has been reported that electrically induced cycling in spinal cord injury patients still leads to increase in HR under control conditions, but not when circulation in the lower limbs was occluded, indicating possible contribution of factors such as hormone concentrations and temperature (FISHER, 2014).

Despite its recognized existence and impact on heart rate variability, the biological relevance and mechanisms behind cardiolocomotor coupling are not completely elucidated (BAILÓN et al., 2013).

\subsubsection{Interactions between cadence, ventilatory frequency, and heart rate}

Many studies demonstrated that not only cardiac rhythm is modulated by ventilatory and locomotor frequencies, but breathing and cadence also coordinate. Such phenomena are thought to reduce energy cost and perception of workload during physical activity (SUMI et al., 2006). Interestingly, the respiratory-to-locomotor 
ratio was independent of the slope, but the phase relation between breathing and step cycle seems to be affected by incline. During uphill running, the onset of inspiration coincided with the support phase, while during downhill running, inspiration started during the floating phase (TAKANO, 1995).

The mechanisms underlying locomotor and ventilatory coupling are not yet completely understood (SUMI et al., 2006).

NIIZEKI et al. (1993) analyzed the interaction between cardiac, ventilatory and locomotor rhythms in subjects running or walking on a treadmill. They showed that when cardiolocomotor coupling was present, the strength of cardiorespiratory coupling was reduced or even absent. This pattern was observed both during spontaneous cardiolocomotor synchronization and when subjects were requested to synchronize cadence and HR. They also found evidence supporting that ventilatory frequency was at least partially modulated by locomotor rhythm on a 1:2 respiration-to-locomotion ratio under free stepping. However, when volunteers had to adjust cadence to HR, the coupling between ventilatory frequency and cadence was disturbed.

\subsubsection{The problem of frequency-overlapping: concluding remarks}

In face of what was presented in the preceding sesstions, it becomes implicit to HRV analysis that a number of diverse inputs tend to, or are able to, modulate the final output of cardiac variability. Methodological limitations and the characteristics of the exercise itself potentially lead to overlapping between cadence and ventilatory rates, mainly under low-intensity activities. As a consequence, it may not be possible to identify the individual contributions of locomotor, ventilatory and autonomic components to the spectral power computed in the HF and LF bands, complicating biological interpretation of HRV under exercise (BLAIN et al., 2009; BAILÓN et al., 2013; ALIKHANI et al., 2017).

In this sense, particular caution should be taken in establishing the definition of the upper limit of the HF band. When HF range is expanded to include frequencies as high as $1 \mathrm{~Hz}$ in order to accommodate the ventilatory frequency under exercise, a 
fraction of the power measured might be associated with the locomotor rate. This overlap leads to an overestimation of the parasympathetic contribution to the HF band power under low-intensity exercise, possibly explaining the high power values reported despite reduced vagal control of HR.

BAILÓN et al. (2013) recommend that both locomotor and ventilatory frequencies to be measured during experiments. Spectral frequencies in which superposition is present should either be excluded from analysis or interpreted with knowledge of such limitations. 


\section{OBJECTIVES AND HYPOTHESIS}

\subsection{OBJECTIVES}

\subsubsection{General objectives}

Running faster or on steepier surfaces potentially represents different stimuli to the cardioventilatory and locomotor systems, particularly regarding metabolic demand, cadence, and ventilatory frequency. These components influence mean and instantaneous heart rate. Therefore, the purpose of the present study is to analyze how heart rate variability responds to changes in velocity and/or slope during running.

\subsubsection{Specific objectives}

Specifically, we seek to address the following questions:

1. How does HR respond to changes in speed and in slope?

2. How does HRV respond to changes in speed and slope and HR? Are the HRV response patterns better explained by $H R$ or by different combinations of speed and slope?

3. Does the HRV variability behave in a similar way to that observed in a homogenous group when a broader range of age and physical fitness are encompassed? 


\subsection{HYPOTHESIS}

1. We hypothesize that heart rate will increase with increasing speed and slope and that this increase will be proportional to the increase in external work calculated accordingly to equation 1 and the study of TAYLOR (1982).

2. Regarding HRV, we expect most of the estimators to be predominantly influenced by metabolic demand, regardless of a given combination of speed and slope. According to previous studies, we expect sdnn, rms and absolute power frequency values of $\mathrm{HF}$ and $\mathrm{LF}$ to decrease as $\mathrm{HR}$ increases. Additionally, we hypothesize that $\mathrm{HF}_{\mathrm{nu}}$ will increase with metabolic demand, reflecting increased breathing frequency, while $L_{\mathrm{nu}}$ decreases. We believe a frequency band associated with cadence will be present throughout all conditions. Finally, as for non-linear analysis, we expect a1ApEn to increase as a function of exercise intensity.

3. We expect HRV variability to present a similar pattern of response to metabolic demand, velocity, and slope when comparing individuals of different ages and physical fitness. 


\section{MATERIALS AND METHODS}

\subsection{SUBJECTS}

In the present study, data from a total of 19 volunteers were analyzed. Subjects engaged in either Experiment $1(N=12)$ or Experiment $2(N=7)$, described below. All volunteers gave written informed consent, and this project was approved by the local ethics committee (CEUA - IBUSP / CAAE 65361317.2.0000.5464). Participating subjects were healthy males without chronic diseases such as diabetes or hypertension or any disease of the cardiovascular and/or locomotor system, including injuries in the past 6 months. All volunteers were trained subjects who engaged in running at least twice a week for a minimum of 1 year and logged 25 or more kilometers per week. A 12 lead clinical electrocardiogram was performed prior to the experiment to ensure subjects had no unknown important cardiac abnormalities.

A restricted population of subjects between the ages of 20 and 45 , and with a specific fitness level, as described below, participated in Experiment 2. Data collected from such group were employed to address questions 1 and 2 .

The group selected for Experiment 2 is homogenous. However, the population that constitutes amateur runners is rather diverse. In order to account of at least part of this diversity, we included a second group, that participated in Experiment 1. This group is composed of individuals of broader fitness spectrum and age (25-yo to 56).

\subsection{DATA ACQUISITION}

All experiments were performed in the Laboratory of Energetics and Theoretical Physiology, at the Department of Physiology, Bioscience Institute, University of São Paulo. Subjects ran on a motorized treadmill at room temperature (average of $21.3^{\circ} \mathrm{C}$, s.d. $=2.3^{\circ} \mathrm{C}$ ) and relative humidity of $62 \%$ (s.d. $=8.7 \%$ ). 
All data was acquired through Biopac Student Lab Pro MP36 (Biopac Systems, Inc.), at a sampling rate of $1000 \mathrm{~Hz}$, using surface electrodes. Electrocardiographic signal was obtained with electrodes in a modified CM5 thoracic configuration. Equipment was set at AHA set up, with a low pass filter of $0.05 \mathrm{~Hz}$ and a high pass of $100 \mathrm{~Hz}$. Electromyographic signal was obtained with electrodes positioned according to SENIAM Project recommendations (HERMENS et al., 1999) in the vastus lateralis (Experiment 1) and vastus medialis (Experiment 2) and gastrocnemius lateralis (Experiments 1 and 2). Equipment was set at EMG configuration with low pass filter of $30 \mathrm{~Hz}$ and high pass at $500 \mathrm{~Hz}$.

\subsection{SPEED SELECTION}

A running velocity $(\mathrm{V})$ was selected for each volunteer to be taken as a reference for calculating experimental velocities. $V$ was chosen as to elicit, at $0 \%$ incline, a heart rate of $77 \%$ to $95 \%$ of the maximum estimated heart rate, corresponding to "vigorous intensity" according to the American College of Sports Medicine (GARBER et al., 2011). Maximum heart rate $\left(H_{\mathrm{max}}\right)$ was estimated according to TANAKA et al. (2001) as:

$$
H R_{\max }=208-0.7 \cdot \text { age in years }
$$

To ensure all volunteers performed the exercise at a comparable relative intensity, only data from those subjects whose HR fell within the goal range of $77 \%$ to $95 \%$ of $\mathrm{HR}_{\max }$ when running at $\mathrm{V}$ speed, $0 \%$ incline were analyzed.

Considering that many runners train on the street or public places, we aimed to analyze a range of slopes compatible with what is most frequently found in such environments. We opted for a incremental protocol (that is, increasing speed, instead of random order or a decremental protocol, for example) as such was demonstrated by NATALI (2015) to be more appropriate in studying HRV. 


\subsection{EXPERIMENTAL PROTOCOL}

Subjects were asked to avoid particularly strenuous training and to abstain from alcohol and caffeine in the $24 \mathrm{~h}$ preceding each experimental session. On experimental days, volunteers were asked to wear appropriate running clothes and shoes. Sessions took place between 7 a.m. - 10 a.m., over the course of 4 weeks or less.

\subsubsection{Experiment 1}

Following electrode placement, subjects performed a 6-minute warm-up (3 minutes walking at $4 \mathrm{~km} / \mathrm{h}$ followed by 3 minutes running at $0.8 \mathrm{~V} \mathrm{~km} / \mathrm{h}$ ). The treadmill was then stopped and eventual adjustments in electrodes or cables were performed. After such procedure, the target runs began.

The target runs were composed of 3 consecutive 6 -minute runs, at $0.8 \mathrm{~V}, 0.9$ $\mathrm{V}$, and $1 \mathrm{~V}$, in this order. Subjects visited the laboratory on 3 different days. Each day, target runs were performed at a different randomly selected slope, $0 \%, 2.5 \%$ or $4 \%$. In total, subjects ran 9 combinations of speed and slope.

\subsubsection{Experiment 2}

The same general organization adopted in Experiment 1 was applied in Experiment 2. Regarding the experimental protocol, the main difference lies in the target runs, which have a broader range of speeds and slopes.

For this protocol, target runs were composed of 4 consecutive 3 minutes runs, also performed at increasing speeds, $0.7 \mathrm{~V}, 0.8 \mathrm{~V}, 0.9 \mathrm{~V}$, and $1 \mathrm{~V}$. The slopes, randomly selected each day, were $0 \%, 2 \%, 4 \%$, and $6 \%$. Only one slope was performed at each visit to the to the laboratory. In total, volunteers that engaged in Experiment 2 performed 16 combinations of speed and slope over the course of 4 non-consecutive days. 


\subsection{DATA PROCESSING}

Signal was processed and analyzed using Matlab R2015b (MathWorks Inc.) and/or $R$ 3.4.3 software ( $R$ Project). The interval between successive $R$ waves in the electrocardiographic signal (RR interval) and successive contractions in the electromyographic signal (instantaneous stride frequency) was obtained. Signals were visually inspected and abnormal beats $(<1 \%)$ were excluded from the records.

The last 15 seconds of each 3 or 6 -minute runs were summarily discarded to avoid effects associated with the transition between conditions. The RR segment analyzed was composed of $256 \mathrm{RR}$ intervals obtained from the last minutes under a given combination of speed and slope. For each record, the following estimators were calculated:

- Time domain: mean HR (HR), standard deviation (sdnn), root mean square of successive differences (rms) and a1ApEn.

- Frequency domain: Fast Fourier Transform was performed, and the resulting spectrum divided in 3 bands, $0.04-0.15 \mathrm{~Hz}$ (LF band), $0.15-0.8 \mathrm{~Hz}$ (HF band, expanded) and $1.3-1.6 \mathrm{~Hz}$. As previously described, we extended the analysis beyond the traditional values of the HF band in order to encompass eventual components associated with ventilatory frequency. The $0.8 \mathrm{~Hz}$ upper limit for HF band was determined according to the typical values for the ventilatory rate under high-intensity exercise (MCARDLE et al, 2006). The upper band $(1.3-1.6 \mathrm{~Hz})$ is the highest possible band allowed by the Nyquist frequency generated by the minimum RR intervals at higher heat rates. It was set as being half the minimum and maximum cadence observed amongst our population, therefore, referred to as $C$ band.

As we performed the FFT analysis on signals composed of 256 RR intervals, corresponding to a few minutes of data collection, we are unable to properly make inferences regarding phenomena that occur at a very low frequency. For that reason, we choose to not discuss results regarding frequencies below $0.04 \mathrm{~Hz}$, which corresponds to the very low frequency (VLF) component of HRV.

Cadence was obtained from the electromyographic signal. 


\subsection{STATISTICAL ANALYSIS}

$H R$, sdnn, rms, and a1ApEn values were made relative, within each individual, to values obtained in the run performed at $0 \%$ incline, $1 \mathrm{~V}$ speed. FFT bands were analyzed as absolute (HF , LF and C) and normalized units (power in a specific band divided by total power per individual, $\mathrm{HF}_{\mathrm{nu}}, \mathrm{LF}_{\mathrm{nu}}$ and $\mathrm{C}_{\mathrm{nu}}$ ). Mean values for the entire population in each given condition were then calculated and used for statistical analysis. Questions 1 and 2 were approached using data collected exclusively from Experiment 2 (a more homogenous population). Question 3 seeks to analyze the impacts on HRV of a broader range of fitness and age, therefore data from Experiment 1 was compared to that of Experiment 2.

Critical p-value was set at 0.05 for all analysis.

Images and gradient analysis were made/calculated using interpolated data. Experimental data were interpolated using the interp2 function in Matlab, with default parameter 'linear'.

Each question was approached as follows:

1. How does HR respond to changes in speed and slope? A multivariable model was fitted to relative $H R$ data $\left(H R_{\text {rel }}\right)$, with speed and slope as independent variables. The model was adjusted as presented in equation 12 , where $Y$ is the HRV estimator, in this case, $\mathrm{HR}_{\mathrm{rel}}$ :

$$
Y=\text { intercept }+a \cdot \text { speed }+b \cdot \text { speed } \cdot \text { slope }
$$

Where speed is given as a fraction of $V$ and slope in percentage. If such model was found to be significant,we proceed to the gradient analysis.

2. How does HRV respond to changes in speed and slope and HR? Are the $H R V$ response patterns better explained by HR or by different combinations of speed and slope? To answer this question, 2 models were fitted to each HRV estimator $(\mathrm{Y})$, a multivariable model with speed and slope as independent variables (as the one used to answer Question 1, equation 12) and a linear model with $H R_{\text {rel }}$ as the independent variable (equation 13). If both models presented significance, they were compared through an ANOVA test. In the 
cases where the two models equally explained the data set, the simplest one was adopted. If the multivariable model was found to better explain our results, we proceeded to analyse the gradient to further elucidate the effects of speed and slope;

$$
Y=\text { intercept }+c \cdot H R_{\text {rel }}
$$

3. Does the HRV variability behave in a similar way to that observed in a homogenous group when a broader range of age and physical fitness are encompassed? The age between experiments were compared using an unpaired t-test, unicaudal. We choose a unicaldal distribution as the lower limit of age was 18 years in both experiments, but the upper limit for Experiment 2 was 45 years while it was unlimited for Experiment 1, therefore we expected mean age of Experiment 1 to be either equal or higher than that of Experiment 2.

The average $\mathrm{V}$ was also compared between experiments through an unpaired t-test, but this time, as we had no expectations a priori, we choose a bicaudal distribution.

In order to verify if the HRV patterns observed in the population from Experiment 2 could explain data from Experiment 1, for each estimator, we predicted what would be expected, given different combinations of speed and slope, if the best-fitted model (according to results from Question 2) was adequate for such population.

We then compared through a paired bicaudal t-test the data we experimentally obtained in Experiment 1 with the predicted according to the model fitted for the population from Experiment 2. For those variables we find the models to be adequate, this analysis ends here. For those variables in which we found the model not to be adequate, we also verified if the error showed a tendency to overestimated/underestimated the variable in question or not, and whether this error was dependent on the conditions (speed and slope or $\mathrm{HR}_{\mathrm{rel}}$ ) used to estimate it. To accomplish this, we observed the ratio between estimated and observed values and performed a linear regression between this ratio and $\mathrm{HR}_{\mathrm{rel}}$ or speed and slope, according to the model. We 
also calculated two models (linear regression of the variable as a function of $\mathrm{HR}_{\text {rel }}$ and a multivariable model with speed and slope as independent variables, as performed in Question 2). This allowed us to establish if the two populations showed the same general pattern. 


\section{RESULTS AND DISCUSSION}

In Tables 2 (Experiment 2) and 3 (Experiment 1), we present the anthropometric characteristics of the volunteers.

Table 2 - Characteristics of volunteers of Experiment 2.

\begin{tabular}{cccccccc}
\hline Subject & $\mathrm{V}(\mathrm{km} / \mathrm{h})$ & $\mathrm{HR}(\mathrm{BPM})$ & $\% \mathrm{HR}_{\max }$ & $\mathrm{HR}_{\max }$ & Age (years) & Height (cm) & Weight $(\mathrm{kg})$ \\
\hline R.S. & 13.0 & 148 & 83 & 179 & 42 & 177 & 84 \\
R.A & 12.0 & 172 & 90 & 191 & 24 & 168 & 79 \\
F.C. & 12.0 & 150 & 84 & 178 & 43 & 163 & 71 \\
D.Q. & 11.0 & 167 & 87 & 193 & 22 & 166 & 53 \\
V.T. & 12.5 & 142 & 77 & 185 & 33 & 172 & 85 \\
C.S. & 11.0 & 166 & 88 & 188 & 29 & 174 & 84 \\
V.Y. & 11.0 & 155 & 83 & 186 & 31 & 185 & 98 \\
mean & 11.8 & 157 & 85 & 186 & 32 & 172 & 79 \\
s.d. & 0.8 & 11 & 4 & 6 & 8 & 7 & 14 \\
\hline is select & & & & & & & \\
\hline
\end{tabular}

$\mathrm{V}$ is selected velocity; $\mathrm{HR}$ is heart rate at $\mathrm{V}$ speed; $\% \mathrm{HR}_{\max }$ is the percentage that $\mathrm{HR}$ at $\mathrm{V}$ speed represents of the maximum estimated $H R ; R_{\text {max }}$ is maximum estimated heart rate.

Table 3 - Characteristics of volunteers of Experiment 1.

(to be continued)

\begin{tabular}{cccccccc}
\hline Subject & $\mathrm{V}(\mathrm{km} / \mathrm{h})$ & $\mathrm{HR}(\mathrm{BPM})$ & $\% \mathrm{HR}_{\max }$ & $\mathrm{HR}_{\max }$ & Age (years) & Height $(\mathrm{cm})$ & Weight $(\mathrm{kg})$ \\
\hline G.C. & 12.0 & 168 & 88 & 191 & 25 & 168 & 60 \\
T.C. & 10.7 & 150 & 80 & 188 & 28 & 175 & 80 \\
C.G. & 10.7 & 157 & 93 & 169 & 56 & 178 & 79 \\
D.N. & 10.0 & 155 & 88 & 176 & 46 & 175 & 73 \\
F.G. & 10.0 & 141 & 82 & 172 & 52 & 171 & 75 \\
E.H. & 14.3 & 147 & 80 & 183 & 36 & 161 & 55 \\
L.B. & 10.2 & 148 & 88 & 169 & 56 & 177 & 70 \\
O.S. & 10.9 & 154 & 86 & 179 & 41 & 170 & 80 \\
R.A. & 14.4 & 158 & 84 & 188 & 29 & 165 & 54 \\
T.V. & 12.0 & 171 & 91 & 188 & 29 & 175 & 73
\end{tabular}


(conclusion)

\begin{tabular}{cccccccc}
\hline Subject & $\mathrm{V}(\mathrm{km} / \mathrm{h})$ & $\mathrm{HR}(\mathrm{BPM})$ & $\% \mathrm{HR}_{\max }$ & $\mathrm{HR}_{\max }$ & Age (years) & Height $(\mathrm{cm})$ & Weight $(\mathrm{kg})$ \\
\hline V.F. & 14.0 & 141 & 81 & 175 & 47 & 176 & 61 \\
W.V. & 16.0 & 154 & 85 & 181 & 39 & 176 & 65 \\
mean & 12.1 & 154 & 86 & 180 & 40 & 172 & 69 \\
s.d. & 2.1 & 9 & 4 & 8 & 11 & 5 & 9.5 \\
\hline
\end{tabular}

$\mathrm{V}$ is selected velocity; $\mathrm{HR}$ is heart rate at $\mathrm{V}$ speed; $\% \mathrm{HR}_{\max }$ is the percentage that $H R$ at $V$ speed represents of the maximum estimated $\mathrm{HR} ; \mathrm{HR}_{\max }$ is maximum estimated heart rate.

Some records had to be excluded from the analysis due to signal noise (in Experiment 1, the $0.9 \mathrm{~V}$ at $2.5 \%$ incline run for E.H.; in Experiment 2, all $4 \%$ incline runs for D.Q., all $2 \%$ incline runs for V.T., the $0.7 \mathrm{~V}$ at $6 \%$ incline run for C.S., and the $0.9 \mathrm{~V}$ at $0 \%$ and $0.8 \mathrm{~V}$ at $6 \%$ runs for $\mathrm{V} . \mathrm{Y}$ ) or volunteer exhaustion (in Experiment 1 , the $1 \mathrm{~V}$ at $4 \%$ run for E.H., and in Experiment 2 the $1 \mathrm{~V}$ at $4 \%$ and $0.9 \mathrm{~V}$ and $1 \mathrm{~V}$ at $6 \%$ for R.S., $0.9 \mathrm{~V}$, and $1 \mathrm{~V}$ at $6 \%$ runs for V.Y. and C.S.).

\subsection{QUESTION 1}

In Table 4 we show absolute and relative HR obtained from Experiment 2. In Fig. 5 we show values of $\mathrm{HR}_{\text {rel }}$ for different combinations of speed and slope and the level curve for such. As expected, the relative heart rate increased with both speed and slope. This result is rather obvious but indicates that the experimental protocol was applied correctly. The multivariable model analysis resulted in equation 14 (associated statistics are presented in Table 5). 
Table 4 - Mean absolute (Hz) and relative HR in Experiment 2, presented as mean (s.d.).

\begin{tabular}{|c|c|c|c|c|c|c|c|c|}
\hline \multirow{3}{*}{$\begin{array}{l}\text { Speed } \\
\text { (fraction } \\
\text { of } \mathrm{V} \text { ) }\end{array}$} & \multicolumn{8}{|c|}{ Slope (\%) } \\
\hline & \multicolumn{4}{|c|}{ Absolute } & \multicolumn{4}{|c|}{ Relative } \\
\hline & 0 & 2 & 4 & 6 & 0 & 2 & 4 & 6 \\
\hline \multirow[t]{2}{*}{0.7} & 2.11 & 2.26 & 2.35 & 2.44 & 0.80 & 0.85 & 0.91 & 0.94 \\
\hline & $(0.27)$ & $(0.25)$ & $(0.22)$ & $(0.19)$ & $(0.06)$ & $(0.07)$ & $(0.07)$ & $(0.04)$ \\
\hline \multirow[t]{2}{*}{0.8} & 2.33 & 2.52 & 2.62 & 2.72 & 0.89 & 0.95 & 1.01 & 1.04 \\
\hline & $(0.25)$ & $(0.27)$ & $(0.22)$ & $(0.18)$ & $(0.04)$ & $(0.07)$ & $(0.05)$ & $(0.05)$ \\
\hline \multirow[t]{2}{*}{0.9} & 2.46 & 2.67 & 2.82 & 2.95 & 0.94 & 1.00 & 1.09 & 1.13 \\
\hline & $(0.25)$ & $(0.25)$ & $(0.18)$ & $(0.13)$ & $(0.03)$ & $(0.07)$ & $(0.06)$ & $(0.07)$ \\
\hline \multirow[t]{2}{*}{1} & 2.62 & 2.83 & 3.00 & 3.10 & 1.00 & 1.07 & 1.15 & 1.19 \\
\hline & $(0.19)$ & $(0.24)$ & $(0.18)$ & $(0.11)$ & $(0.00)$ & $(0.07)$ & $(0.08)$ & $(0.09)$ \\
\hline
\end{tabular}

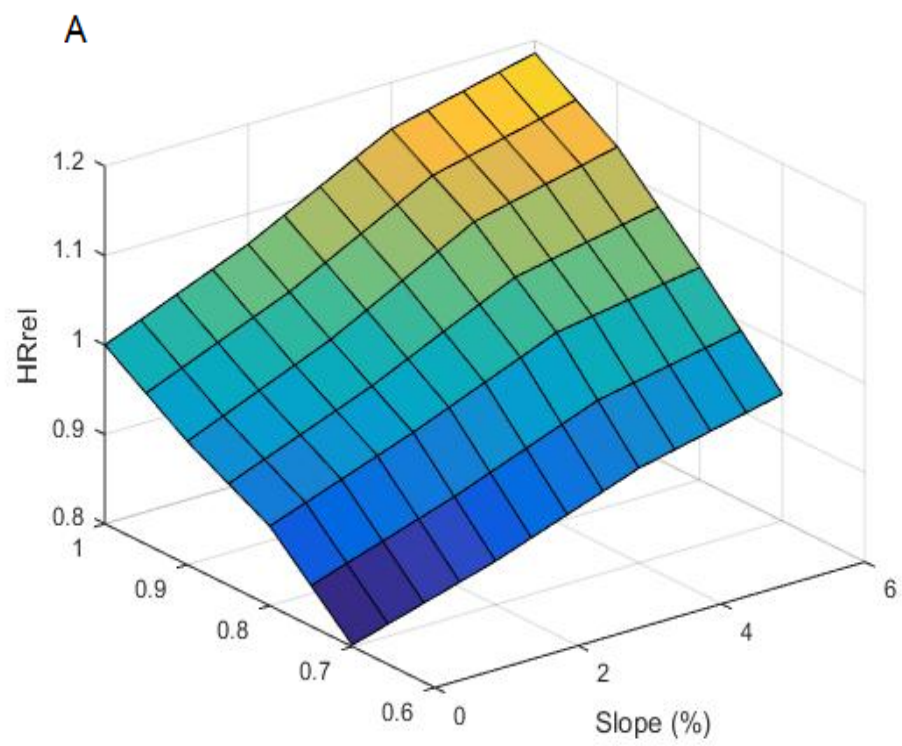

B

Speed (fraction of V)

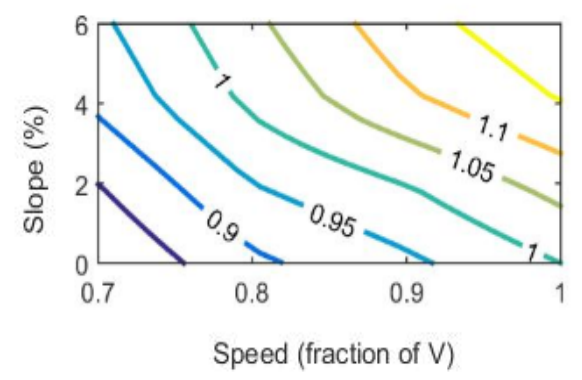

Figure 5 - Relative (according to the $0 \%$ incline, $V$ speed run) (A) HR in each combination of speed and incline and (B) it's associated level curve. 


$$
H R_{\text {rel }}=0.36+0.643 \cdot \text { speed }+0.033 \cdot \text { speed } \cdot \text { slope }
$$

Table 5 - Statistical details for equation 14.

\begin{tabular}{lrrr}
\hline & error & \multicolumn{2}{c}{ p-value } \\
\hline intercept & 0.03 & $<0.001$ & \\
speed coef. & 0.03 & $<0.001$ & \\
interaction & 0.001 & $<0.001$ & \\
coef. & & & \\
& p-value & adjusted r & F \\
\hline model & $<0.001$ & 0.98 & 398.40 \\
\hline
\end{tabular}

\subsubsection{What is the equivalence of speed and slope regarding HR?}

Equation 14 allows us to estimate that an increment in $0.1 \mathrm{~V}$ leads to an increase of $0.065+0.0033$ slope in relative HR while increasing slope by $1 \%$ leads to a 0.033 speed increase. The pattern of colors in Fig. 5 of estimated/measured $H R_{\text {rel }}$ (painel A) and the level curve (painel B) allow us to visualize the estimated $H R_{\text {rel }}$ for each combinations of speed and slope, as well as which conditions result in the same expected $\mathrm{HR}_{\mathrm{rel}}$.

We applied the same analytical process to the data presented by JONES \& DOUST(1996) (Table 1) and PADULO et al. (2013). For the work of JONES \& DOUST(1996), values of both HR and speed were made relative to the run performed at $0 \%$ incline, $4.58 \mathrm{~m} / \mathrm{s}$. As explained previously, such condition was selected as the subjects presented a HR corresponding to $84 \%$ of estimated maximum $H R$, therefore, it was the combination of speed and slope that most resembles ours (V speed, 0\% incline). As for PADULO et al. (2013), speed was constant throughout the experiment (set at $15 \mathrm{~km} / \mathrm{h}$ ), therefore, values were made relative to those observed when running at $0 \%$ slope. 
We fitted a multivariable model with the same structure of the one in equation 14 (that is, relative $H R$ as a function of speed + speed slope) to the data from JONES \& DOUST(1996). Results show $\left(F=757\right.$, adjusted $r^{2}=0.98, p$-value $\left.<0.001\right)$ that increasing slope by $1 \%$ leads to a 0.035 speed increment in relative $H R$ (standard error $=0.003, p$-value $<0.001$ ), while increasing speed by $0.1 \mathrm{~V}$ results in a increment in relative HR of $0.068+0.035$ slope (standard error $=0.002, p<0.001$ ). When analyzing data obtained from PADULO et al. (2013) using a multivariable model of the same structure we find $\left(F=363\right.$, adjusted $\left.r^{2}=0.99, p=0.03\right)$ that $1 \%$ increment in the slope leads to a 0.021 increase in relative HR (standard error = $0.001, p=0.03$ )

In all studies, the run used as basis corresponded to $80-90 \%$ of maximum heart rate, therefore, relative exercise intensity was approximately similar. Absolute speed, on the other hand, was not. Mean speed for the reference run in our study was $11.8 \mathrm{~km} / \mathrm{h}$, while in data from PADULO et al. (2013) speed was $15 \mathrm{~km} / \mathrm{h}$, and $16.5 \mathrm{~km} / \mathrm{h}$ in the work of JONES \& DOUST(1996). Therefore, it is safe to assume that the fitness level of the participants engaging in each study was notably distinct. Nevertheless, the resulting models from the three data sets are fairly similar.

\subsubsection{Does HR changes similarly along different running conditions?}

In Figs. 6 and 7, we present the magnitude and angle of changes in estimated relative HR obtained in Experiment 2.

When looking at Fig. 6, some aspects bring attention: First, as slope increases, the magnitude of changes in $\mathrm{HR}_{\text {rel }}$ increases. Second, as exercise intensity increases, the magnitude in $\mathrm{HR}_{\text {rel }}$ decreases. Possible reasons for such pattern are discussed below.

If we look at both the magnitude (Fig. 6) and angle (Fig. 7) simultaneously we will notice that the higher magnitudes are associated with the higher angles, or, in other words, as HR changes more, the impact of slope declines, and speed becomes increasingly important. 
A

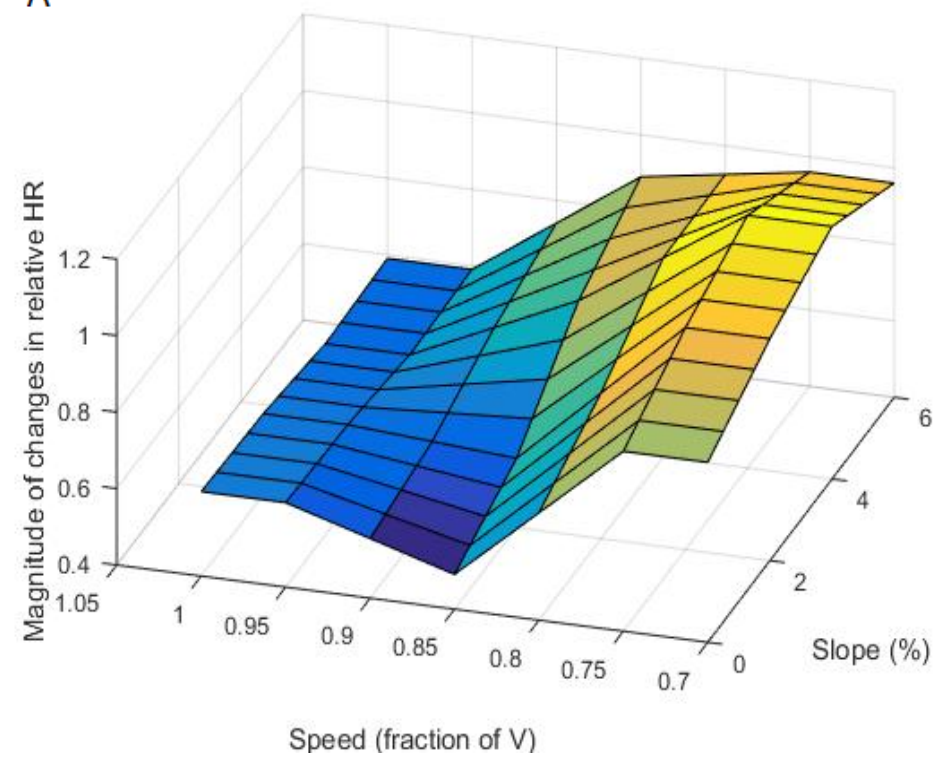

B

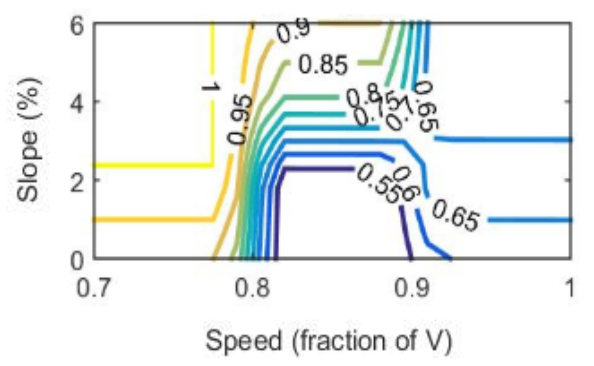

Figure 6 - (A) The magnitude of changes in relative HR based on data obtained in Experiment 2 and (B) it's associated level curve.

A

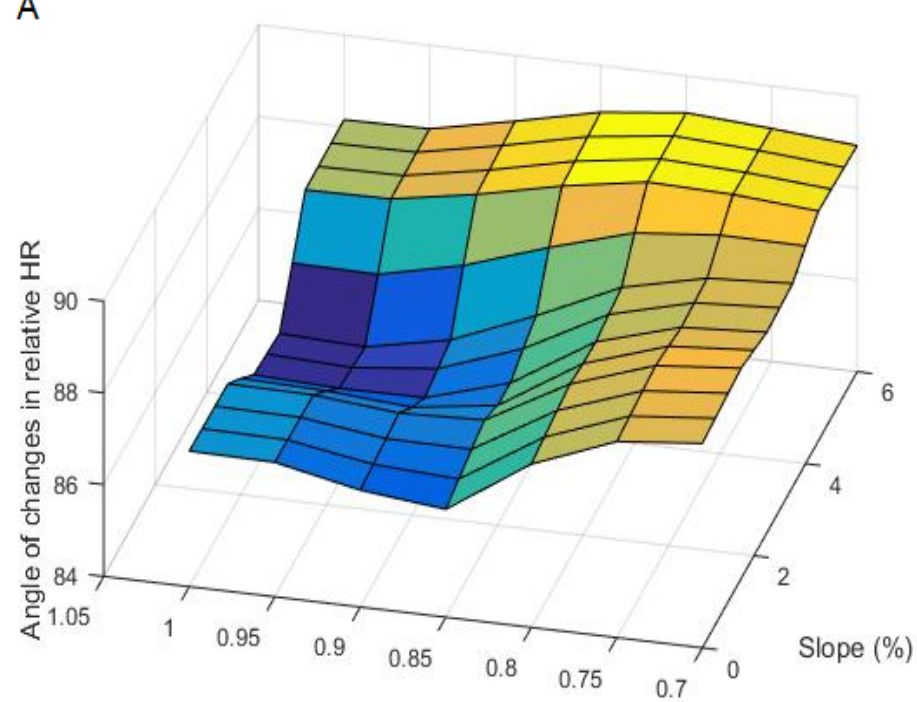

Speed (fraction of $\mathrm{V}$ )
B

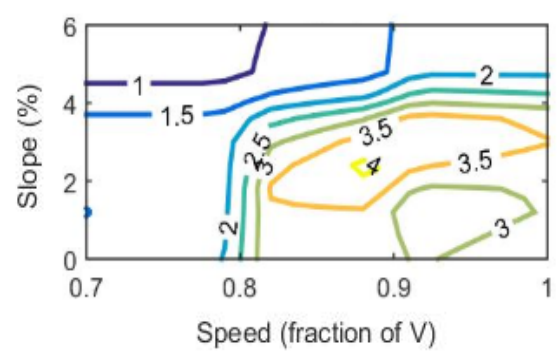

Figure 7 - (A) The angle of changes in relative HR based on data obtained in Experiment 2 and (B) it's associated level curve.

In 1982, TAYLOR et al. published a paper on the relationship between metabolic energy consumption, body size, and speed. They combined experimental and previously reported data of a total of 62 mammalian and avian species so to 
establish an "allometric equation relating mass-specific rates of oxygen consumption using locomotion at a constant speed to speed and body mass". Measures were performed with animals running for 15 - 30 minutes, in order to assure a steady-state condition. Blood lactate and respiratory quotient were measured to assure that the animals performed the exercise under a predominantly aerobic metabolism. They have reached the equation 15 :

$$
\frac{E}{M}=10.7 \cdot M^{-0.316} \cdot v+6.03 \cdot M^{-0.303}
$$

Where $E$ is energy consumption in watts, $M$ is mass in $\mathrm{kg}$, and $v$ is velocity in $\mathrm{m} / \mathrm{s}$. Authors also compared this equation with those formulated separately for each taxonomic group and verified that all of them fell within the $95 \%$ confidence interval of this general equation.

Rearranging equation 15 and adding a component associated with the increase in the body's center of mass present when running uphill (equation 1), we have equation 16 :

$$
E=10.7 \cdot M^{0.68} \cdot v \cdot \cos (a)+g \cdot M v \cdot \operatorname{sen}(a)+6.03 \cdot M^{0.7}
$$

We calculated the magnitude of changes in energy consumption estimated by equation 16 and present the results in Fig. 8. If increments in relative HR were to follow equation 16, and assuming exercise was performed at an intensity in which heart rate is directly and linearly proportional to energy consumption, we should observe a similar pattern in Figs. 6 and 8.

Let us see what happens in Fig. 8 when we compare the changes in speed observed at different slopes. We notice that, for example, variations in speed when at $0 \%$ incline, presents the same magnitude of change in metabolic demand for all speeds (all values are equal, dark blue). The same happens at all different slopes, all magnitude values, regardless of the speed, are the same within a slope. This shows that, for a given slope, metabolic demand changes linearly with speed, as previously reported (MINETI et al., 2002; VERNILLO et al., 2017)

Now, still looking at Fig. 8, let us fix speed and see at what happens as slope increases. We should notice that when running at higher slopes, the changes in metabolic demand are of a bigger magnitude. This is associated with that fact that 
when running on a positive slope, part of the energy expenditure is due to the elevation of the body's center of mass. As we express slope in percent values, there is linearity between the increase in the body's center of mass in the vertical axis and slope, therefore, the higher the magnitude.

There are some similarities between Fig. 8, based on equation 16, and Fig. 6 , based on our experimental data, such as an approximately linear increase in magnitude with increasing slope. This is in accordance with data reanalyzed from MINETTI et al. (2002), equation 3. Still, it is clear that $\mathrm{HR}_{\text {rel }}$ behaves in a different pattern than the one predicted by equation 16 . This might indicate that either (1) metabolic demand did not follow the prediction and/or (2) the premise of linearity between relative metabolic demand and relative HR was not met (at least under some runs).

As previously described, the biomechanics of running on level ground and uphill are distinct, which certainly affects the cost of locomotion, regardless of changes in the body's center of mass. The reduced capacity to store and release elastic energy as slope increases (VERNILLO et al, 2017), for example, would lead to a less efficient run on steepier surfaces, causing a mismatch between metabolic demand predicted according to equation 16 (which considers the vertical component of the movement as behaving such as a block being pushed uphill) and observed in real subjects.

Regarding the relationship between $H R$ and metabolic demand, HR, like all biological processes, presents saturation. As HR increases and approaches its maximum value, the rate of increment decreases (MCARDLE et al., 2006). During our experiments, subjects attained very high HR values (some even above estimated maximum). This should explain the decrease in the magnitude of change when approaching maximum exercise intensity.

The angle of changes observed in our data (Fig. 7) and predicted according to equation 16 (Fig. 9), bear a striking resemblance. This indicates that the relative impact of speed and slope follow the predicted by equation 16.

One way to look at the angles is drawing a line from the $0.7 \mathrm{~V}$ speed $0 \%$ slope run to $1 \mathrm{~V}$ speed, $6 \%$ slope run. As we move towards small speed-high slope combinations, the importance of speed increases (angles approach 90 degrees and 
its maximum values, yellow). The analogous is valid when moving towards the highest speed-smallest slope combination, angles reach its minimum values (blue), indicating that the impact of slope on relative HR/energy consumption increases. When both speed and slope are either high or low, the responsiveness of relative $\mathrm{HR} / \mathrm{energy}$ consumption to each of those factors is intermediate (green).

All angles are quite close to 90 degrees. That is because of the units we choose to use for speed and slope. Imagine a runner whose selected $V$ speed was $10 \mathrm{~km} / \mathrm{h}$. We don't need much analysis to imagine that going from $7 \mathrm{~km} / \mathrm{h}(0.7 \mathrm{~V})$ to $14 \mathrm{~km} / \mathrm{h}(1.4 \mathrm{~V})$ has a much bigger impact on HR than increasing slope from $1 \%$ to $2 \%$.

We considered the component associated with slope as only the increase in body's center of mass, which is obviously a simplification, as it does not consider peculiarities of human movement. Still, despite this "oversimplification", experimental data are similar to predicted by equation 16 , which means that indeed the elevation in body's center of mass must be the most important component associated with the increase in metabolic demand when running uphill. This is in accordance with VERNILLO et al. (2007).

It is also interesting to note that equation 16 was based on terrestrial mammals and avians, not particularly humans, yet, it fits experimental data. Therefore, the most important factors associated with cost of running must be body mass, speed and slope. If on one hand this conclusion is rather obvious, on the other hand it is noteworthy that, roughly, the characteristics of a particular pattern on movement (stride frequency, joint movement, even bipedalism) are not necessary to explain our data.

Our results disagree with the angles calculated using data from JONES \& DOUST(1996). Data from those authors indicate that the higher the slope, the more sensitive $\mathrm{HR}_{\mathrm{rel}}$ is to it (smaller angles) (see "Introduction"). The reasons behind such discrepancies are unknown to the authors. Regarding magnitude values, the range obtained using data from the aforementioned paper falls within that obtained by us for the same slopes. The more dramatic difference in slope effect observed in our study is most likely associated with the larger range of slopes used. 


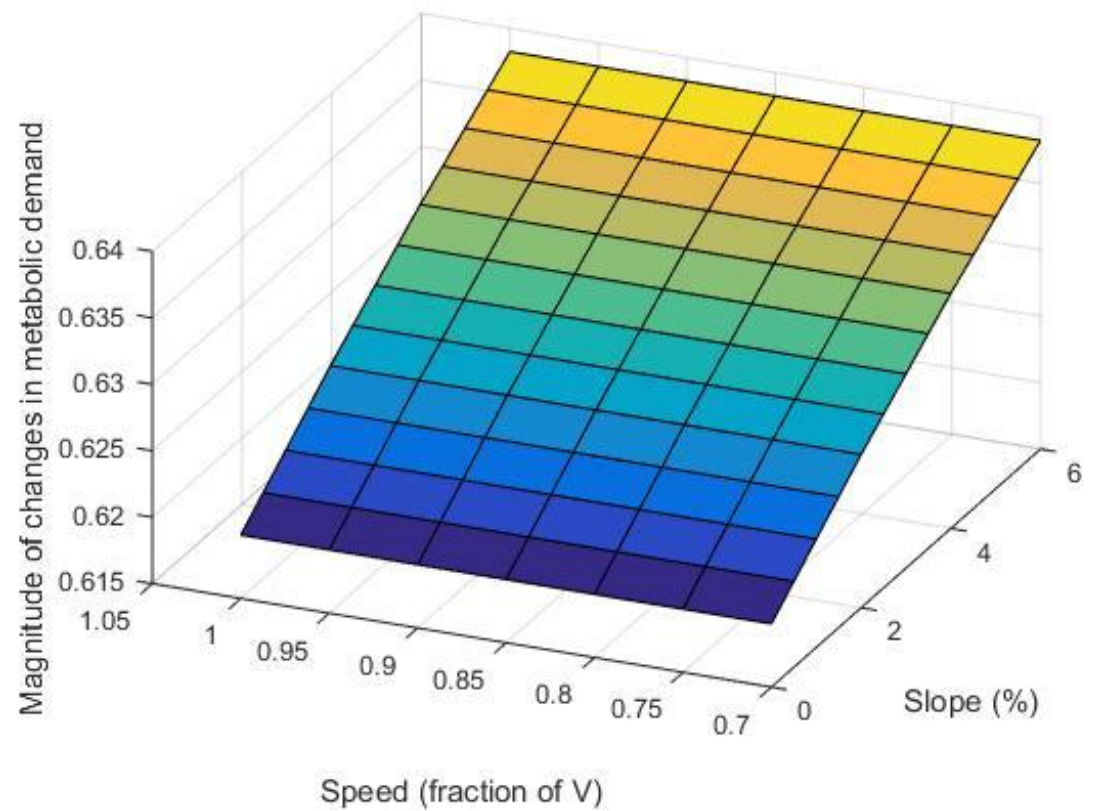

Figure 8 - The magnitude of changes in $E$ (metabolic demand) estimated through equation 16.

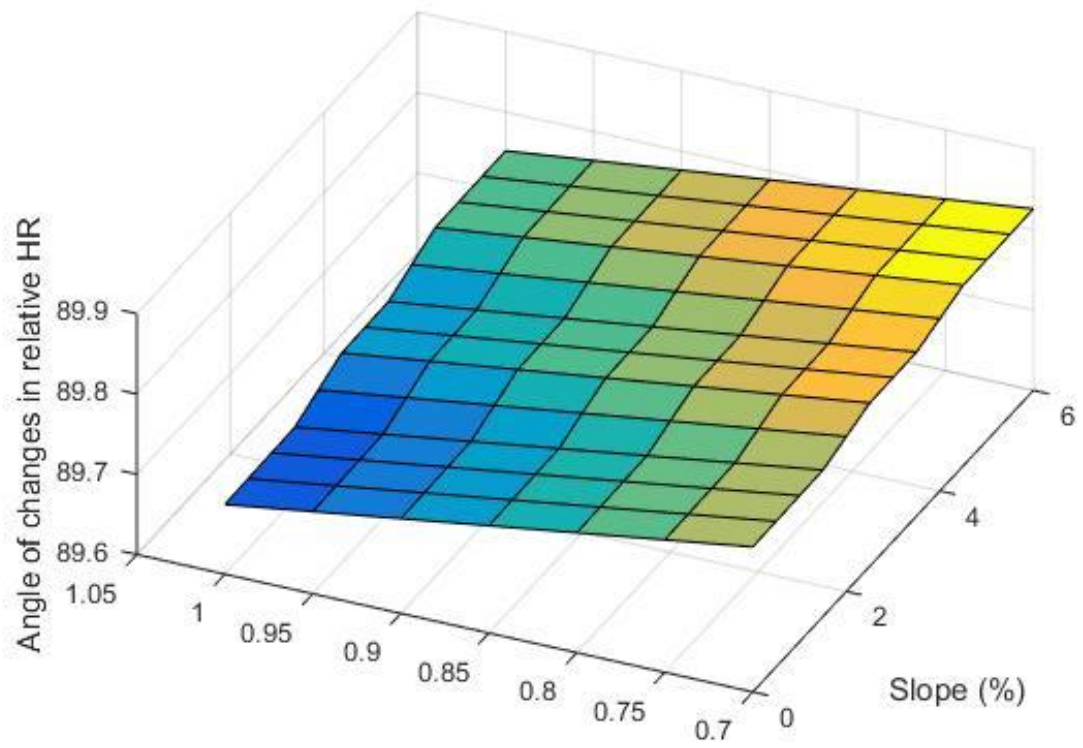

Speed (fraction of $\mathrm{V}$ )

Figure 9 - The angle of changes in $\mathrm{E}$ (metabolic demand) estimated through equation 16 


\subsubsection{In conclusion...}

We found that an increment in $0.1 \mathrm{~V}$ leads to an increase of $0.065+0.0033$. slope in relative HR while increasing slope by $1 \%$ leads to a 0.033 - speed increase. Additionally, we found that the angle of the gradient of the changes in $\mathrm{HR}_{\text {rel }}$ behaves as would be expected by the general metabolic rate equation reported by TAYLOR et al. (1982) for mammalian and avian species, plus a component associated with an increase in the body's center of mass when running uphill. The magnitude of the gradient of changes, on the other hand, despite some similarities with the predicted values, such as an approximately linear relationship between HR and slope, could not be fully explained by such equations. Loss of linearity between metabolic demand and HR most likely play a role in such discrepancy.

\subsection{QUESTION 2}

\subsubsection{Time domain estimators}

\subsubsection{The standard deviation of RR intervals (sdnn)}

In Table 6 we present the mean and standard deviation of the absolute and relative sdnn obtained in Experiment 2. In Fig. 10 are shown experimental and interpolated sdnn rel values for the different combination of speed and slope.

The multivariable model analysis resulted in equation 17 (associated statistics are presented in Table 7). A second model was fitted, with $s^{2} n_{\text {rel }}$ as a function of $\mathrm{HR}_{\mathrm{rel}}$, regardless of the combinations of speed and slope (equation 18, associated statistics are presented in Table 8). 
Table 6 - Mean absolute $\left(s \cdot 10^{-2}\right)$ and relative sdnn in Experiment 2, presented as mean (s.d.).

\begin{tabular}{|c|c|c|c|c|c|c|c|c|}
\hline \multirow{3}{*}{$\begin{array}{l}\text { Speed } \\
\text { (fraction } \\
\text { of } V \text { ) }\end{array}$} & \multicolumn{8}{|c|}{ Slope (\%) } \\
\hline & \multicolumn{4}{|c|}{ Absolute } & \multicolumn{4}{|c|}{ Relative } \\
\hline & 0 & 2 & 4 & 6 & 0 & 2 & 4 & 6 \\
\hline \multirow[t]{2}{*}{0.7} & 1.59 & 1.13 & 1.22 & 1.07 & 3.44 & 2.64 & 2.35 & 2.44 \\
\hline & $(1.05)$ & $(0.55)$ & $(0.49)$ & $(0.19)$ & $(3.05)$ & $(1.72)$ & $(1.34)$ & (1.05) \\
\hline \multirow[t]{2}{*}{0.8} & 0.66 & 0.47 & 0.39 & 0.37 & 1.28 & 1.07 & 0.75 & 0.71 \\
\hline & $(0.27)$ & $(0.09)$ & $(0.19)$ & $(0.14)$ & $(0.48)$ & $(0.43)$ & $(0.45)$ & $(0.11)$ \\
\hline \multirow[t]{2}{*}{0.9} & 0.63 & 0.47 & 0.28 & 0.29 & 1.42 & 1.09 & 0.53 & 0.58 \\
\hline & $(0.38)$ & $(0.18)$ & $(0.06)$ & $(0.11)$ & $(1.14)$ & $(0.56)$ & $(0.17)$ & $(0.17)$ \\
\hline \multirow[t]{2}{*}{1} & 0.55 & 0.29 & 0.32 & 0.25 & 1.00 & 0.63 & 0.55 & 0.54 \\
\hline & $(0.23)$ & $(0.06)$ & $(0.09)$ & $(0.07)$ & $(0.00)$ & $(0.17)$ & $(0.12)$ & $(0.18)$ \\
\hline
\end{tabular}

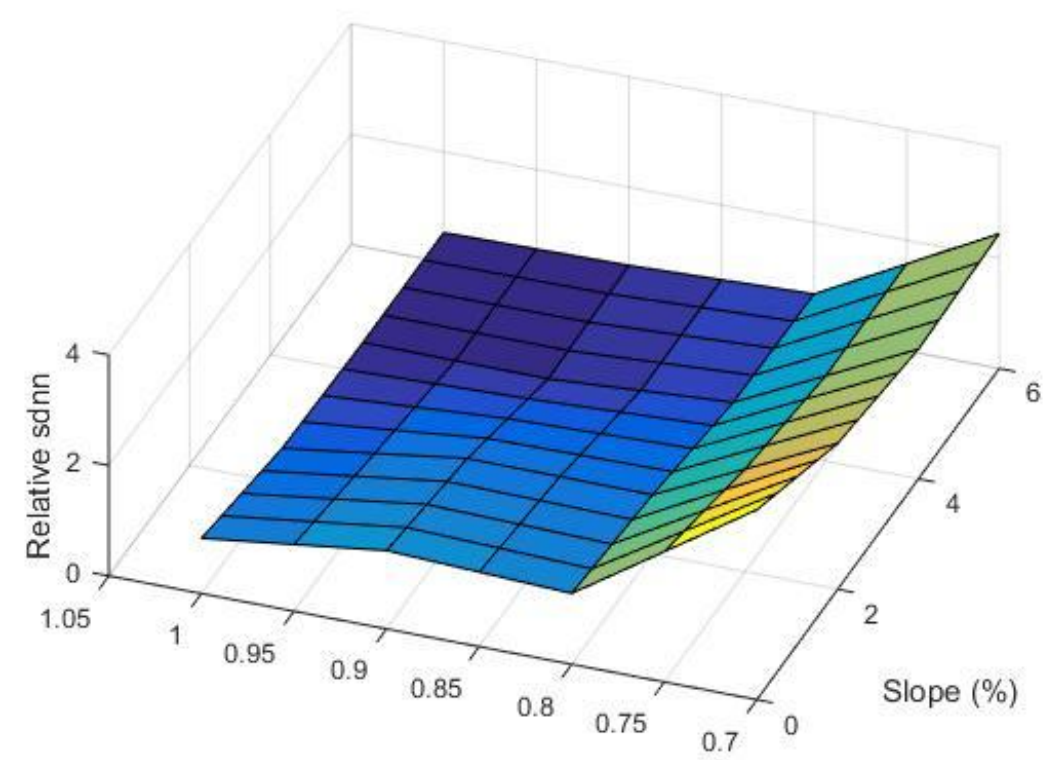

Speed (fraction of $\mathrm{V}$ )

Figure 10 - Relative sdnn for each combination of speed and incline.

$s d n n_{\text {rel }}=6.55-5.74 \cdot$ speed $-0.14 \cdot$ speed $\cdot$ slope 
Table 7 - Statistical details for equation 17.

\begin{tabular}{lrrr}
\hline & error & \multicolumn{2}{c}{$p$-value } \\
\hline intercept & 1.01 & $<0.001$ & \\
speed coef. & 1.19 & $<0.001$ & \\
interaction & 0.06 & 0.06 & \\
coef. & & & \\
& p-value & adjusted r $r^{2}$ & $F$ \\
\hline model & $<0.001$ & 0.66 & 15.72 \\
\hline
\end{tabular}

$s d n n_{r e l}=8.31-7.02 \cdot H R_{r e l}$

Table 8 - Statistical details for equation 18.

\begin{tabular}{lrrr}
\hline & error & \multicolumn{2}{c}{ p-value } \\
\hline intercept & 1.15 & $<0.001$ & \\
$\mathrm{HR}_{\text {rel }}$ coef. & 1.15 & $<0.001$ & \\
& p-value & adjusted $\mathrm{r}^{2}$ & $\mathrm{~F}$ \\
\hline model & $<0.001$ & 0.71 & 37.66 \\
\hline
\end{tabular}

As can be observed by comparing equations 17 and 18, the coefficients are negative, either those multiplying speed, slope or $\mathrm{HR}_{\text {rel. }}$ As there is a clear association between increasing speed or slope and $\mathrm{HR}_{\mathrm{rel}}$, coefficients with the same signal were expected.

We compared the 2 models, equations 17 and 18, and present the results in Table 9. As the simplest model $\left(\mathrm{sdnn}_{\text {rel }}\right.$ as a function of $\left.\mathrm{HR}_{\text {rel }}\right)$ also has the smaller residual sum of squares (RSS), we considered it to be better suited to explain our data. Hence, we conclude that in our experiments, $s d n n_{\text {rel }}$ can be better explained by $H R_{\text {rel }}$. 


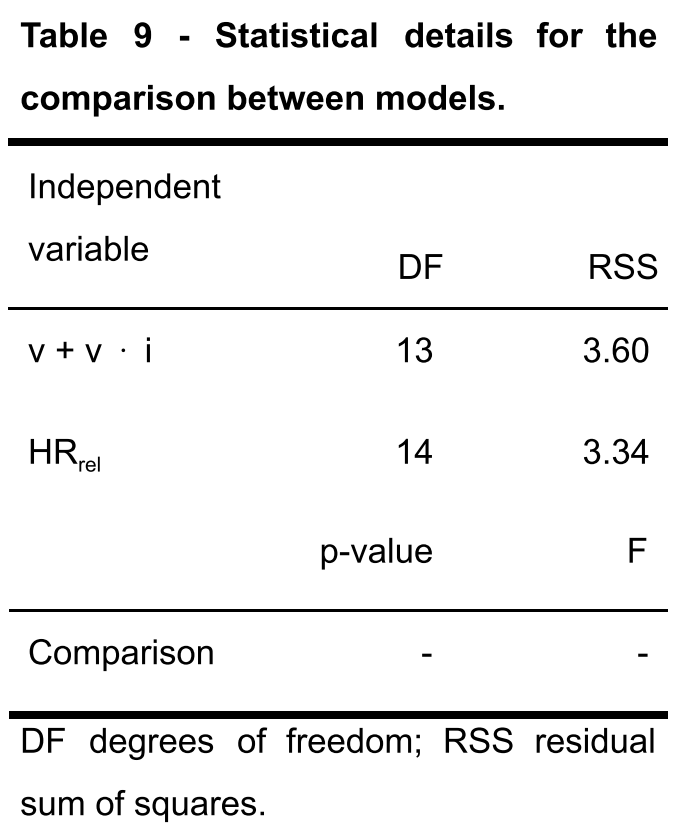

The absolute sdnn values obtained in our experiments (15.9 to $2.5 \mathrm{~ms}$ ) are well within previously reported data for exercising at a heart rate of 160 BPM or more, that is, between 3 to $10 \mathrm{~ms}$ (MICHAEL et al., 2017).

Many studies reported that sdnn (or total spectral power) decreases with exercise intensity (PERINI \& VEICSTEINAS, 2003; CARTER et al., 2003), and exercise intensity seems to be the most important factor affecting HRV (MICHAEL et al., 2017). Such decrement in sdnn values with exercise intensity occurs in a curvilinear shape. While some studies suggest that minimum sdnn values are reached at moderate-high intensity exercise, others advocate that beyond such intensity sdnn continues to decrease, but at lower rates (MICHAEL et al., 2017). Although a linear regression was enough to explain our data, as can be observed in Fig. 10 , changes in $s \mathrm{dnn}_{\text {rel }}$ are of a bigger magnitude at lower intensity runs in comparison with its changes at higher intensity runs. These varying rates of changes points in favor of a curvilinear decrease in sdnn. 
4.2.1.2 Root mean square of successive differences of RR intervals ( $r m s$ )

In Table 10 we present the mean and standard deviation of the absolute and relative rms obtained for Experiment 2 and its relative values in Fig. 11.

The multivariable model analysis resulted in equation 19 (associated statistics are presented in Table 11). A second model was fitted, with $\mathrm{rms}_{\mathrm{rel}}$ as a function of $\mathrm{HR}_{\text {rel}}$, regardless of the combination of speed and slope (equation 20, associated statistics are presented in Table 12).

Table 10 - Mean absolute (ms) and relative rms for Experiment 2, presented as mean (s.d.).

\begin{tabular}{|c|c|c|c|c|c|c|c|c|}
\hline \multirow{3}{*}{$\begin{array}{c}\text { Speed } \\
\text { (fraction } \\
\text { of } V \text { ) }\end{array}$} & \multicolumn{8}{|c|}{ Slope (\%) } \\
\hline & \multicolumn{4}{|c|}{ Absolute } & \multicolumn{4}{|c|}{ Relative } \\
\hline & 0 & 2 & 4 & 6 & 0 & 2 & 4 & 6 \\
\hline \multirow[t]{2}{*}{0.7} & 5.40 & 3.95 & 3.52 & 3.12 & 1.67 & 1.22 & 1.06 & 0.97 \\
\hline & (3.81) & $(0.89)$ & $(0.70)$ & $(0.86)$ & (1.19) & $(0.42)$ & $(0.39)$ & $(0.24)$ \\
\hline \multirow[t]{2}{*}{0.8} & 3.70 & 3.35 & 2.96 & 2.74 & 1.12 & 1.01 & 0.87 & 0.78 \\
\hline & $(0.29)$ & $(0.54)$ & $(0.47$ & $(0.85)$ & $(0.21)$ & $(0.18)$ & $(0.13)$ & $(0.11)$ \\
\hline \multirow[t]{2}{*}{0.9} & 3.56 & 3.17 & 2.95 & 2.58 & 1.05 & 0.92 & 0.86 & 0.79 \\
\hline & $(0.50)$ & $(0.96)$ & $(0.57)$ & $(0.63)$ & $(0.12)$ & $(0.11)$ & $(0.12)$ & $(0.08)$ \\
\hline \multirow[t]{2}{*}{1} & 3.40 & 3.25 & 3.40 & 2.90 & 1.00 & 0.95 & 0.93 & 0.88 \\
\hline & $(0.73)$ & $(0.99)$ & $(1.01$ & $(0.74)$ & $(0.00)$ & $(0.09)$ & $(0.16)$ & $(0.09)$ \\
\hline
\end{tabular}




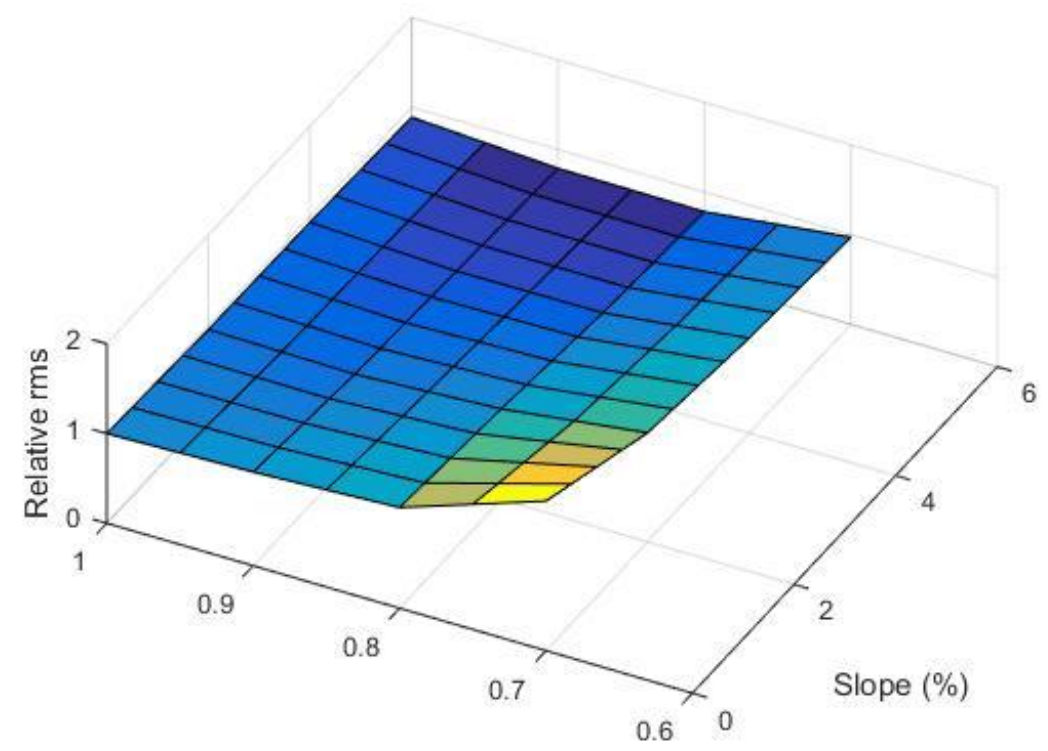

Speed (fraction of $\mathrm{V}$ )

Figure 11 - Relative rms for each combination of speed and incline.

$r m s_{\text {rel }}=1.77-0.72 \cdot$ speed $-0.06 \cdot$ speed $\cdot$ slope

Table 11 - Statistical details for equation 19.

\begin{tabular}{lccc}
\hline & error & p-value & \\
\hline intercept & 0.28 & $<0.001$ & \\
speed coef. & 0.34 & 0.05 & \\
interaction & 0.02 & 0.01 & \\
coef. & & & \\
& p-value & adjusted r & $\mathrm{F}$ \\
\hline model & $<0.01$ & 0.51 & 8.69 \\
\hline
\end{tabular}

$r m s_{r e l}=2.49-1.49 \cdot H R_{r e l}$ 
Table 12 - Statistical details for equation 20.

\begin{tabular}{lrrr}
\hline & error & \multicolumn{2}{c}{ p-value } \\
\hline intercept & 0.32 & $<0.001$ & \\
HR $_{\text {rel }}$ coef. & 0.32 & $<0.001$ & \\
& p-value & adjusted $\mathrm{r}^{2}$ & $\mathrm{~F}$ \\
\hline model & $<0.001$ & 0.57 & 21.22 \\
\hline
\end{tabular}

We compared the 2 models, equations 19 and 20, and present the results in Table 13. As the simplest model $\left(\mathrm{rms}_{\mathrm{rel}}\right.$ as a function of $\left.H \mathrm{R}_{\mathrm{rel}}\right)$ also has the smaller RSS, we considered it to be better suited to explain our data. Hence, like observed for the $\mathrm{sdnn}_{\text {rel }}$ estimator, $\mathrm{rms}_{\text {rel }}$ is inversely proportional to metabolic demand and can be better explained by $\mathrm{HR}_{\mathrm{rel}}$ instead of speed and slope. The resemblance between those two time domain estimators had been pointed out by Michael et al. (2017).

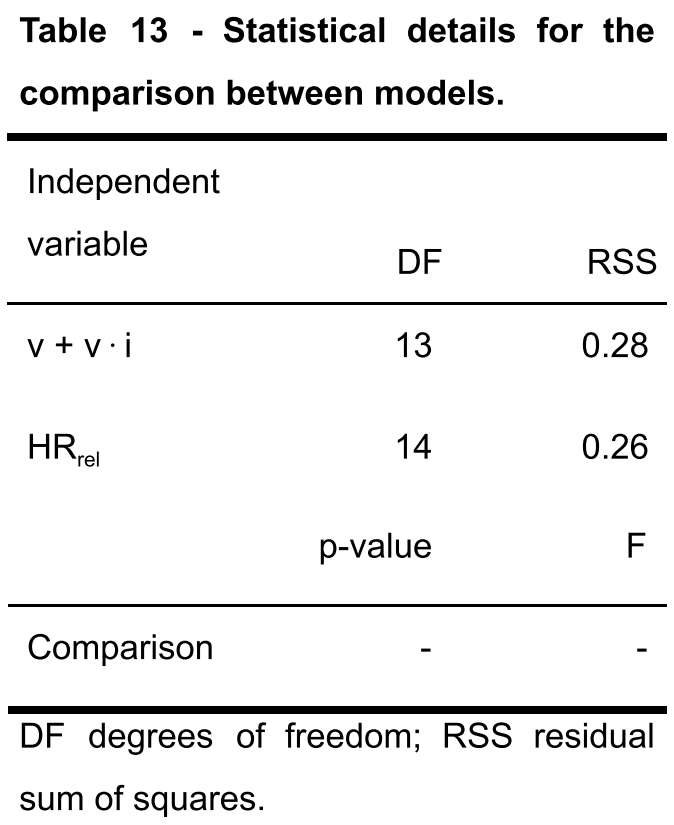

rms had also been shown to present a curvilinear decay with increasing exercise intensity. We notice that at lower exercise intensities, $\mathrm{rms}_{\text {rel }}$ changes more drastically when compared to intermediate-higher exercise intensities (Fig. 11). Compared to sdnn, minimum values (lower than $5 \mathrm{~ms}$ ) are attained at a more 
moderate exercise intensity (HR of 120 to $140 \mathrm{BPM}$ or 50 to $60 \% \mathrm{VO}_{2 \max }$ ) (MICHAEL et al., 2017). This might explain the general resemblance observed by comparing Figs. 10 and 11 and also why the range of relative rms is smaller than relative sdnn.

The exercise intensity in which rms attains its minimal values coincides with the first ventilatory threshold. The fact that absolute rms values obtained in our experiments ranged from 5.5 to 2.9 ms indicates that most of our volunteers ran at or beyond such physiological marker. Slight increases in rms have been reported at HR above 180 BPM (MICHAEL et al., 2017). We did not observe such pattern.

\subsubsection{Area 1 of Approximate Entropy (a1ApEn)}

We present the absolute and relative values of a1ApEn obtained in Experiment 2 in Table 14 and Fig. 12. The multivariable model analysis resulted in equation 21 (associated statistics are presented in Table 15). A second model was fitted, with a1ApEn $n_{\text {rel }}$ as a function of $\mathrm{HR}_{\text {rel, }}$, regardless of the combination between speed and slope (equation 22, associated statistics are presented in Table 16).

Table 14 - Mean absolute and relative a1ApEn in Experiment 2, presented as mean (s.d.).

\begin{tabular}{|c|c|c|c|c|c|c|c|c|}
\hline \multirow{3}{*}{$\begin{array}{c}\text { Speed } \\
\text { (fraction } \\
\text { of } V \text { ) }\end{array}$} & \multicolumn{8}{|c|}{ Slope (\%) } \\
\hline & \multicolumn{4}{|c|}{ Absolute } & \multicolumn{4}{|c|}{ Relative } \\
\hline & 0 & 2 & 4 & 6 & 0 & 2 & 4 & 6 \\
\hline \multirow[t]{2}{*}{0.7} & 0.14 & 0.16 & 0.14 & 0.12 & 0.55 & 0.62 & 0.55 & 0.50 \\
\hline & $(0.05)$ & $(0.08)$ & $(0.04)$ & $(0.03)$ & $(0.22)$ & $(0.32)$ & $(0.15)$ & $(0.23)$ \\
\hline \multirow[t]{2}{*}{0.8} & 0.23 & 0.27 & 0.25 & 0.27 & 0.91 & 1.01 & 1.02 & 1.03 \\
\hline & $(0.05)$ & $(0.05)$ & $(0.05)$ & $(0.04)$ & $(0.18)$ & $(0.24)$ & $(0.20)$ & $(0.13)$ \\
\hline \multirow[t]{2}{*}{0.9} & 0.24 & 0.26 & 0.30 & 0.29 & 0.94 & 1.01 & 1.18 & 1.06 \\
\hline & $(0.08)$ & $(0.07)$ & $(0.04)$ & $(0.08)$ & $(0.39)$ & $(0.38)$ & $(0.15)$ & $(0.17)$ \\
\hline \multirow[t]{2}{*}{1} & 0.26 & 0.31 & 0.33 & 0.32 & 1.00 & 1.18 & 1.27 & 1.21 \\
\hline & $(0.05)$ & $(0.04)$ & $(0.04)$ & $(0.05)$ & $(0.00)$ & $(0.22)$ & $(0.26)$ & $(0.28)$ \\
\hline
\end{tabular}




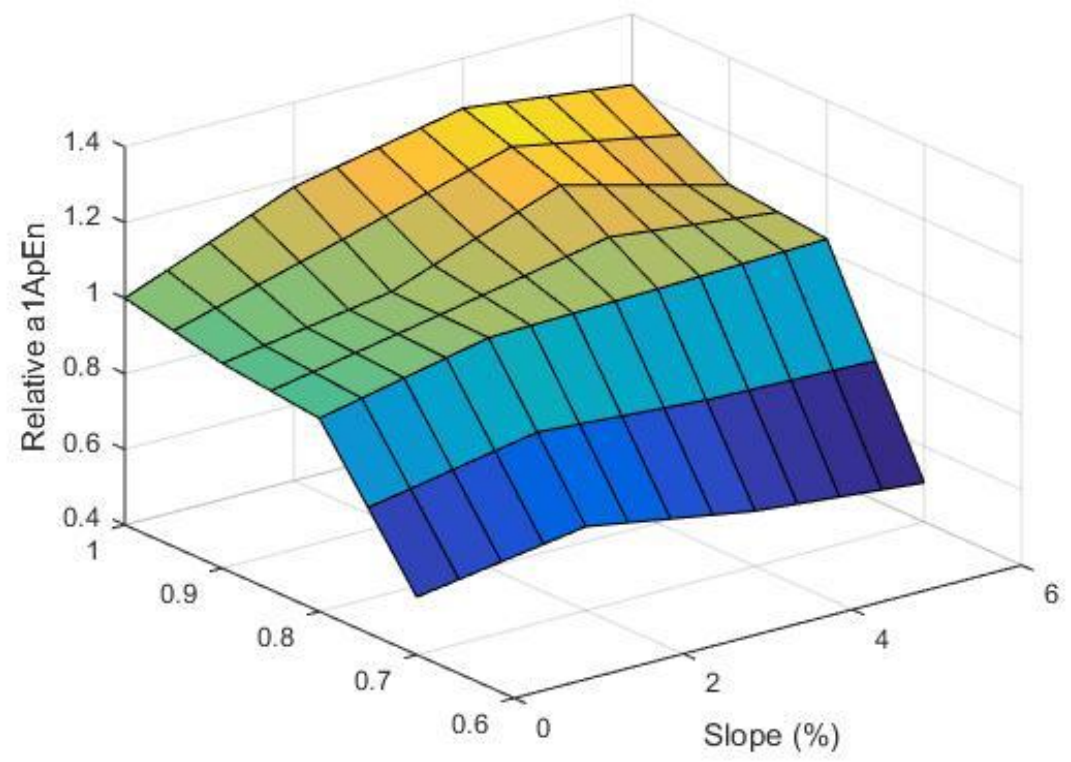

Speed (fraction of V)

Figure 12 - Relative a1ApEn for each combination of speed and incline.

$a 1 A p E n_{\text {rel }}=-0.66+1.8 \cdot$ speed $+0.023 \cdot$ speed $\cdot$ slope

Table 15 - Statistical details for equation 21

\begin{tabular}{lccc}
\hline & error & p-value & \\
\hline intercept & 0.24 & $<0.01$ & \\
speed coef. & 0.28 & $<0.001$ & \\
interaction & 0.02 & 0.18 & \\
$\begin{array}{l}\text { coef. } \\
\text { p-value }\end{array}$ & adjusted r & $\mathrm{F}$ \\
\hline model & $<0.001$ & 0.75 & 24.36 \\
\hline
\end{tabular}

$a 1 A p E n_{r e l}=-0.95+1.89 \cdot H R_{r e l}$ 
Table 16 - Statistical details for equation 22.

\begin{tabular}{lrrr}
\hline & error & \multicolumn{2}{c}{ p-value } \\
\hline intercept & 0.33 & $<0.01$ & \\
HR $_{\text {rel }}$ coef. & 0.33 & $<0.001$ & \\
& p-value & adjusted r & $F$ \\
\hline model & $<0.001$ & 0.68 & 32.83 \\
\hline
\end{tabular}

We compared the 2 models, equations 21 and 22, and present the results in Table 17. Comparing the RSS values of the models and $p$-value of the comparison between them, we conclude that the first equation, with both speed and slope as independent variables, is the one that best explains our data

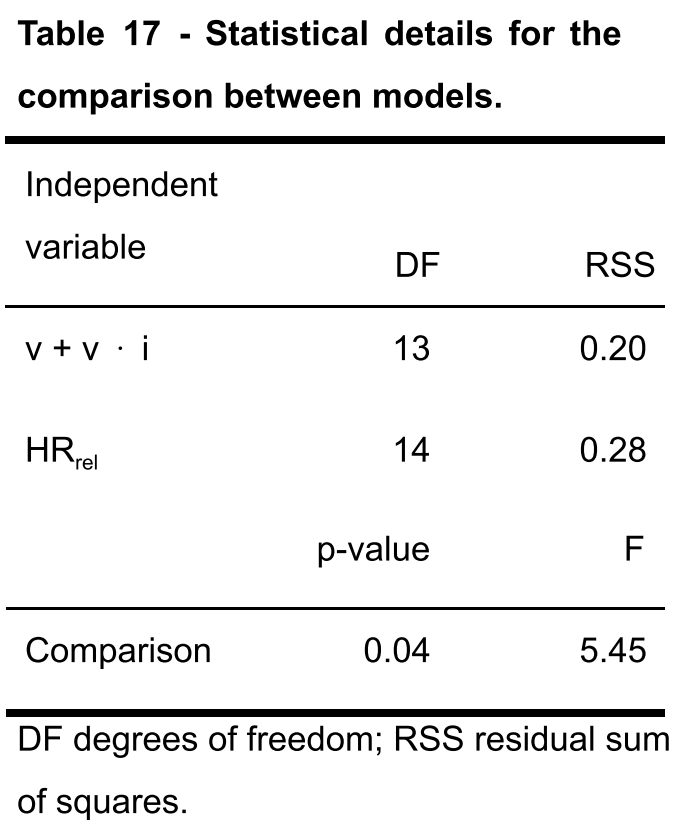

TULPPO et al. (1996) studied HRV, including ApEn, behavior during the parasympathetic blockade at rest and followed by physical activity. Exercise protocol consisted of an incremental test on a cycle ergometer. They reported little variations of ApEn during atropine administration at rest. During exercise, at intensities higher than the ventilatory threshold, ApEn values progressively increased, both in the control group and in those submitted to parasympathetic blockage. Such results were 
interpreted as an indication that ApEn is not influenced by vagal tone, and that sympathetic stimulation leads to higher complexity as measured by such estimator. PERKIOMAKI et al. (2002) also analyzed the effect of atropine administration in HRV measured through ApEn and reached a similar conclusion, that ApEn is not influenced by parasympathetic tone.

Our results also indicate a directly proportional relationship between exercise intensity and a1ApEn, in accordance with the aforementioned studies. It is worth recalling that our protocol was performed most likely at an intensity close to the second ventilatory threshold, and, thus, vagal stimulation of the heart is presumed to be nonexistent or of little importance.

However, not all studies have identified a directly proportional relationship between HRV entropy and exercise intensity. LEWIS \& SHORT (2007) quantified SampEn (an entropy estimator derived from ApEn) during controlled and spontaneous breathing at rest and during progressive exercise on a cycle ergometer. They failed to observe an increment in SampEn as the exercise intensity increased. Actually, there was a tendency for SampEn to decrease as power increased, although this tendency did not reach statistical significance.

The discrepancy when comparing the results obtained by TULPPO et al. (1996) and LEWIS \& SHORT (2007) could hardly be explained by differences in protocol, as both studies were incremental protocols performed on a cycle ergometer at a constant cadence. LEWIS \& SHORT (2007) also observed no differences in SampEn when comparing spontaneous and controlled breathing, indicating that the breathing pattern is not a determinant of entropy values.

The physiological interpretation of nonlinear estimators is not completely established and the effects of workload on HRV entropy are still controversial, as exemplified in the previous paragraphs. ApEn (TULPPO et al., 1996; TULPPO et al., 2001; PERKIOMAKI et al., 2002;) and SampEn (LEWIS \& SHORT, 2007) presented either a weak or no correlation with other HRV linear estimators or spectral measures.

One of the most interesting aspects observed in our study regarding the behavior of a1ApEn is that it is sensitive to different running conditions, not only $\mathrm{HR}_{\text {rel. }}$. We should bring to attention that in the multivariable model only the coefficient 
multiplying speed reached significance level, while the one associated with the interaction speed-slope did not. This indicates that velocity is the most relevant component affecting a1ApEn $\mathrm{n}_{\text {rel }}$ in the model tested.

As can be observed in Fig. 12, a1ApEn $n_{\text {rel }}$ values increase with speed. Values are relatively low at lower speeds for all inclines. At speeds higher than $0.8 \mathrm{~V}$ values of $a 1 A p E n_{\text {rel }}$ increase in what seems to be a different rate. This may be further appreciated looking at the magnitude of such changes in Fig. 13. The magnitude of changes in $a 1 A p E n_{\text {rel }}$ drops dramatically from 0.7 to $0.8 \mathrm{~V}$ speed. Above such velocity, they remain relatively constant. The angle is presented on Fig. 14.

The influence of running conditions on a1ApEn leads us to think that such estimator might be sensitive to peripheral afferents modulating $H R$, such as mechanical impact associated with stride frequency.

It is also worth pointing out that the pattern in $a 1 A p E n_{\text {rel }}$ resembles those seen in processes that reach a saturation, such as observed for HR and the other time domain estimators, indicating that during our experiments volunteers are probably approaching maximum a1ApEn $\mathrm{n}_{\text {rel }}$.

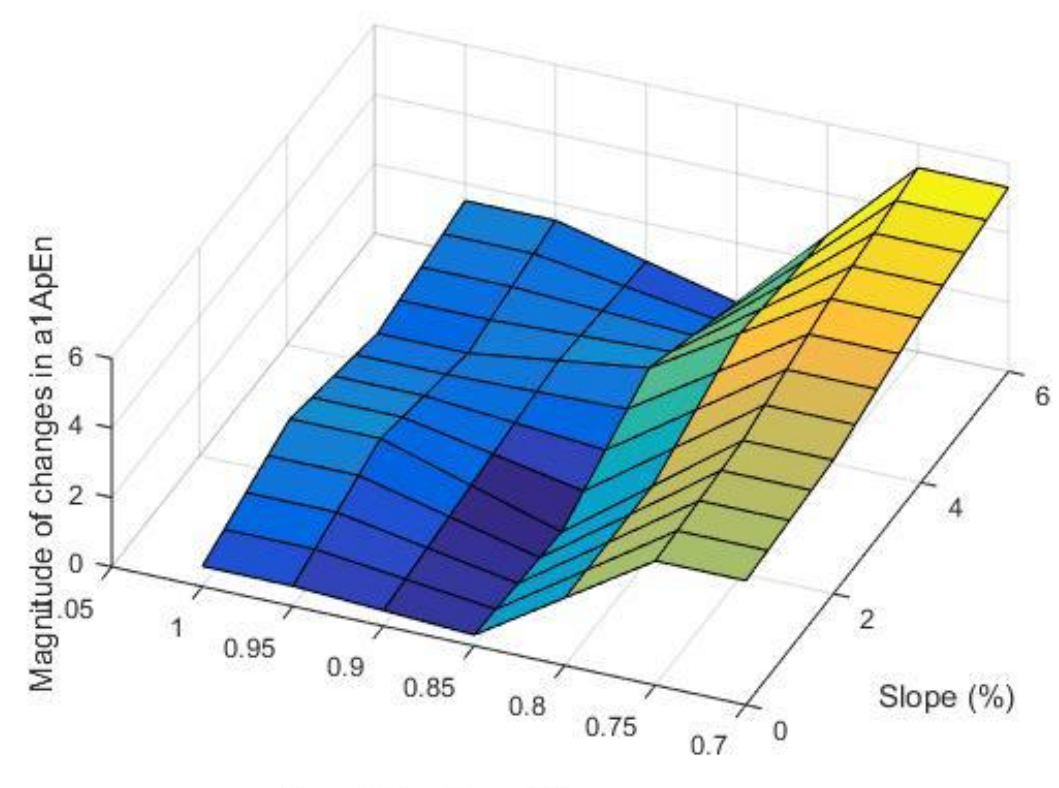

Speed (fraction of V)

Figure 13 - The magnitude of changes in $a 1 \mathrm{ApEn}_{\text {rel }}$ based on data obtained in Experiment 2. 


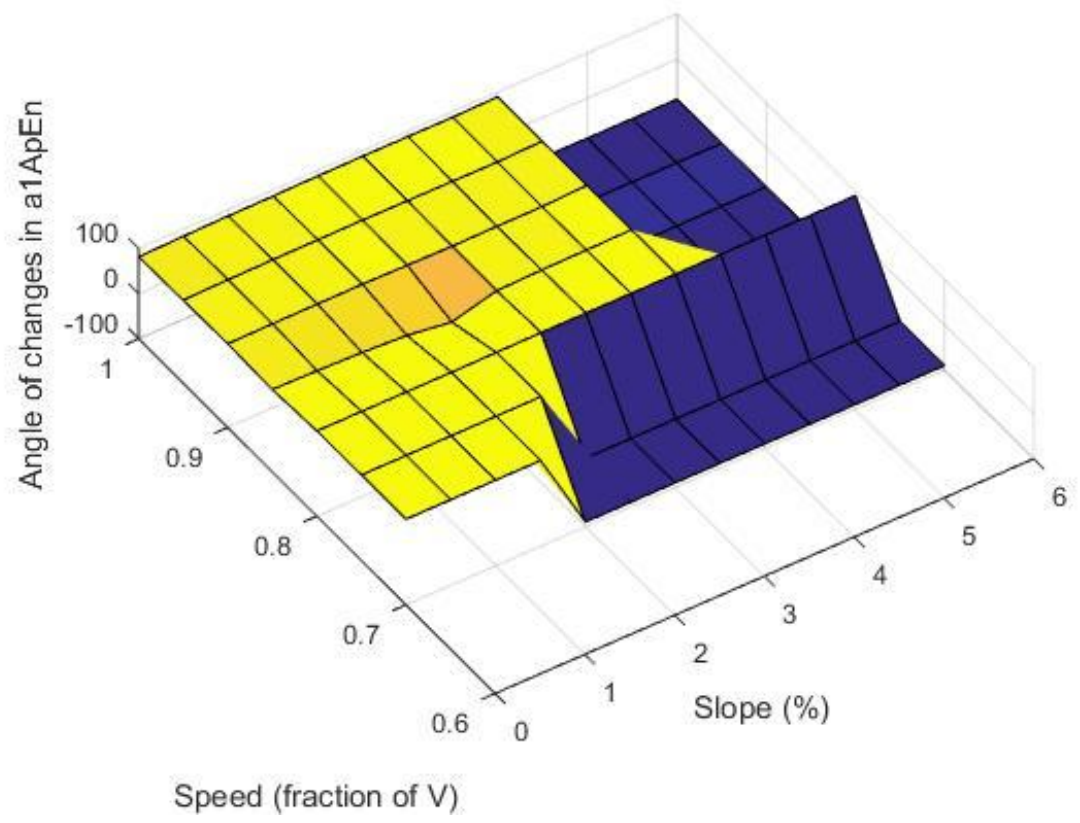

Figure 14 - The angle of changes in a1 $A p E n_{\text {rel }}$ based on data obtained in Experiment 2.

Few studies address the impact of cadence on HRV entropy. NATALI (2015) found a positive correlation between a1ApEn and speed while running. However, in the aforementioned study, we are unable to separate possible effects associated with cadence and metabolic demand, as increments in speed, which likely results in changes in cadence are bound to energy expenditure. Still, such a positive correlation does not disagree with our findings. The relationship between cadence and a1ApEn might be an interesting area for future studies, particularly under conditions of similar metabolic demand and drastically different stride frequencies.

We can understand the responsiveness of $\mathrm{a} 1 \mathrm{ApEn} \mathrm{n}_{\text {rel }}$ to speed and slope by looking at the angle of changes (Fig. 14.) As the angle approaches 90 degrees (positive or negative) the impact of speed on a1ApEn $n_{\text {rel }}$ increases. Negative values indicate that the changes promoted by speed and slope are in the opposite directions (one tends to increase $\mathrm{a} 1 \mathrm{ApEn}$ rel, the other tends to decrease it). Under all conditions a1ApEn $n_{\text {rel }}$ responds mostly to speed. Still, when the slope is relatively high compared to speed (either combination of high slope or intermediate slope associated with low speed), angles shift from positive to negative values. This might indicate a1ApEn presents a biphasic response to the slope, increasing its value under some 
conditions and decreasing under others. This hypothesis would be in accordance with the lack of linearity found between a1ApEn and the coefficient multiplying slope.

\subsubsection{Frequency domain estimators}

The discussion regarding LF and HF bands will be presented after results from all these frequency bands are shown. The same applies for $\mathrm{C}$ band.

\subsubsection{LF band $(0.04-0.15 \mathrm{~Hz})$}

We present the absolute and normalized power in the LF band (Table 18).

Table 18 - Mean absolute $\left(s^{2} / \mathrm{Hz}\right)$ and normalized power in $\mathrm{LF}$ band in Experiment 2 presented as mean (s.d.).

\begin{tabular}{|c|c|c|c|c|c|c|c|c|}
\hline \multirow{3}{*}{$\begin{array}{c}\text { Speed } \\
\text { (fraction } \\
\text { of } \mathrm{V} \text { ) }\end{array}$} & \multicolumn{8}{|c|}{ Slope (\%) } \\
\hline & \multicolumn{4}{|c|}{ Absolute $\left(\cdot 10^{-5}\right)$} & \multicolumn{4}{|c|}{ Normalized } \\
\hline & 0 & 2 & 4 & 6 & 0 & 2 & 4 & 6 \\
\hline \multirow[t]{2}{*}{0.7} & 5.80 & 4.08 & 2.15 & 2.53 & 0.199 & 0.245 & 0.187 & 0.227 \\
\hline & $(9.82)$ & $(4.81)$ & $(1.14)$ & $(1.27)$ & $(0.104)$ & $(0.127)$ & $(0.084)$ & $(0.088)$ \\
\hline \multirow[t]{2}{*}{0.8} & 1.63 & 0.40 & 0.26 & 0.40 & 0.255 & 0.166 & 0.133 & 0.217 \\
\hline & $(2.68)$ & $(0.26)$ & $(0.25)$ & $(0.42)$ & $(0.201)$ & $(0.058)$ & $(0.071)$ & $(0.153)$ \\
\hline \multirow[t]{2}{*}{0.9} & 1.23 & 0.33 & 0.18 & 0.22 & 0.219 & 0.111 & 0.145 & 0.183 \\
\hline & $(1.62)$ & $(0.33)$ & $(0.16)$ & $(0.23)$ & $(0.113)$ & $(0.044)$ & $(0.074)$ & $(0.101)$ \\
\hline \multirow[t]{2}{*}{1} & 0.53 & 0.14 & 0.17 & 0.16 & 0.179 & 0.122 & 0.105 & 0.151 \\
\hline & $(0.38)$ & $(0.13)$ & $\left(0.15^{6}\right)$ & $(0.21)$ & $(0.097)$ & $(0.106)$ & $(0.051)$ & $(0.141)$ \\
\hline
\end{tabular}




\subsection{Absolute power}

In order to verify if absolute power decreased as exercise intensity increased, we adjusted a linear regression with $\mathrm{HR}_{\text {rel }}$ as the independent variable and absolute LF power as the dependent variable. As expected, LF power decreased with increasing heart rate $(p-v a l u e<0.001)$.

\subsection{Normalized power}

We present the normalized LF band $\left(\mathrm{LF}_{\mathrm{nu}}\right)$ in Fig. 15 The multivariable model analysis resulted in equation 23 (associated statistics are presented in Table 19). A second model was fitted, with $L_{\mathrm{nu}}$ as a function of $\mathrm{HR}_{\mathrm{rel}}$, regardless of the combination between speed and slope (equation 24, associated statistics are presented in Table 20).

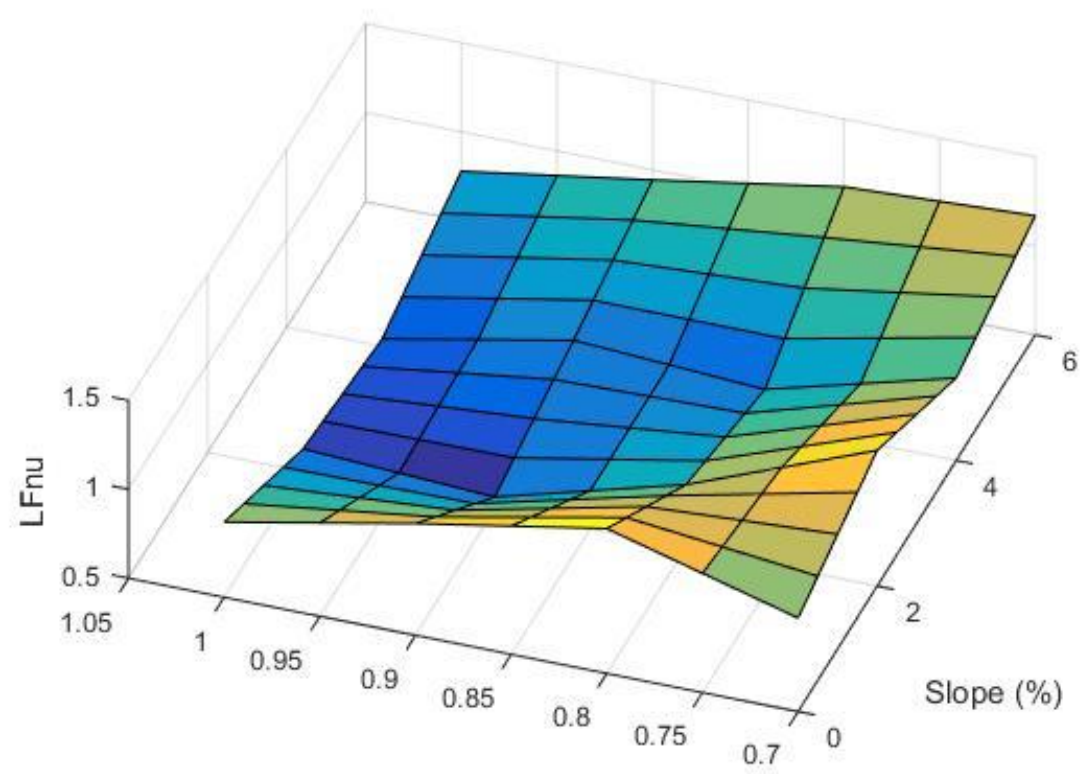

Speed (fraction of V)

Figure $15-\mathrm{LF}_{\mathrm{nu}}$ for each combination of speed and incline.

LFnu $=0.39-0.24 \cdot$ speed $-0.004 \cdot$ speed $\cdot$ slope 
Table 19 - Statistical details for equation 23.

\begin{tabular}{lccc}
\hline & error & $p$-value \\
\hline intercept & 0.07 & $<0.001$ & \\
speed coef. & 0.09 & 0.02 & \\
interaction & 0.005 & 0.38 & \\
coef. & & & \\
& $p-v a l u e$ & adjusted $r^{2}$ & \\
\hline model & 0.03 & 0.33 & 4.73 \\
\hline
\end{tabular}

$L F n u=0.45-0.27 \cdot H R_{r e l}$

Table 20 - Statistical details for equation 24.

\begin{tabular}{lccc} 
& error & p-value & \\
\hline intercept & 0.09 & $<0.001$ & \\
HR $_{\text {rel }}$ coef. & 0.09 & 0.008 & \\
& & & \\
& p-value & adjusted ${ }^{2}$ & $\mathrm{~F}$ \\
\hline model & 0.008 & 0.36 & 9.39 \\
\hline
\end{tabular}

We compared the 2 models, equations 23 and 24, and present the results in Table 21. As both models equally explain LF $_{n u}$, we selected the simplest model, with only $\mathrm{HR}_{\text {rel }}$ as the predictor. 
Table 21 - Statistical details for the comparison between models.

\begin{tabular}{lrr}
\hline $\begin{array}{l}\text { Independent } \\
\text { variable }\end{array}$ & DF & RSS \\
\hline $\mathrm{v}+\mathrm{v} \cdot \mathrm{i}$ & 13 & 0.02 \\
$\mathrm{HR}$ & 14 & 0.02 \\
& $\mathrm{p}$-value & $\mathrm{F}$ \\
\hline Comparison & 0.44 & 0.51 \\
\hline $\begin{array}{l}\text { DF degrees of freedom; RSS } \\
\text { sum of squares. }\end{array}$ &
\end{tabular}

\subsubsection{2 $\mathrm{HF}$ band $(0.15-0.8 \mathrm{~Hz})$}

We present the absolute and normalized power in HF band (Table 22).

Table 22 - Mean absolute $\left(\mathrm{s}^{2} / \mathrm{Hz}\right)$ and normalized power in $\mathrm{HF}$ band in Experiment 2 presented as mean (s.d.).

\begin{tabular}{|c|c|c|c|c|c|c|c|c|}
\hline \multirow{3}{*}{$\begin{array}{c}\text { Speed } \\
\text { (fraction } \\
\text { of } V \text { ) }\end{array}$} & \multicolumn{8}{|c|}{ Slope (\%) } \\
\hline & \multicolumn{4}{|c|}{ Absolute $\left(\cdot 10^{-5}\right)$} & \multicolumn{4}{|c|}{ Normalized } \\
\hline & 0 & 2 & 4 & 6 & 0 & 2 & 4 & 6 \\
\hline \multirow[t]{2}{*}{0.7} & 2.67 & 1.16 & 0.72 & 0.72 & 0.081 & 0.106 & 0.093 & 0.073 \\
\hline & $(4.92)$ & $(1.00)$ & $(0.47)$ & $(0.50)$ & $(0.042)$ & $(0.034)$ & $(0.055)$ & $(0.020)$ \\
\hline \multirow[t]{2}{*}{0.8} & 0.47 & 0.32 & 0.28 & 0.23 & 0.152 & 0.166 & 0.260 & 0.187 \\
\hline & $(0.16)$ & $(0.17)$ & $(0.18)$ & $(0.20)$ & $(0.092)$ & $(0.041)$ & $(0.167)$ & $(0.101)$ \\
\hline \multirow[t]{2}{*}{0.9} & 0.45 & 0.27 & 0.20 & 0.09 & 0.133 & 0.184 & 0.255 & 0.183 \\
\hline & $(0.46)$ & $(0.14)$ & $(0.13)$ & $(0.11)$ & $(0.050)$ & $(0137)$ & $(0.131)$ & $(0.134)$ \\
\hline \multirow[t]{2}{*}{1} & 0.25 & 0.23 & 0.13 & 0.11 & 0.107 & 0.274 & 0.192 & 0.189 \\
\hline & $(0.09)$ & $(0.15)$ & $(0.14)$ & $(0.19)$ & $(0.052)$ & $(0.150)$ & $(0.117)$ & $(0.143)$ \\
\hline
\end{tabular}




\subsubsection{Absolute power}

In order to verify if absolute power decreased as exercise intensity increased, we adjusted a linear regression with $\mathrm{HR}_{\text {rel }}$ as the independent variable and absolute HF power as the dependent variable. As expected, HF power decreased ( $p$-value < $0.001)$ with increasing heart rate.

\subsubsection{Normalized power}

We present the normalized $\mathrm{HF}$ band $\left(\mathrm{HF}_{\mathrm{nu}}\right)$ in Fig. 16. The multivariable model analysis resulted in equation 25 (associated statistics are presented in Table 23). A second model was fitted, with $\mathrm{HF}_{\mathrm{nu}}$ as a function of $\mathrm{HR}_{\text {rel }}$, regardless of the combination between speed and slope (equation 26, associated statistics are presented in Table 24).

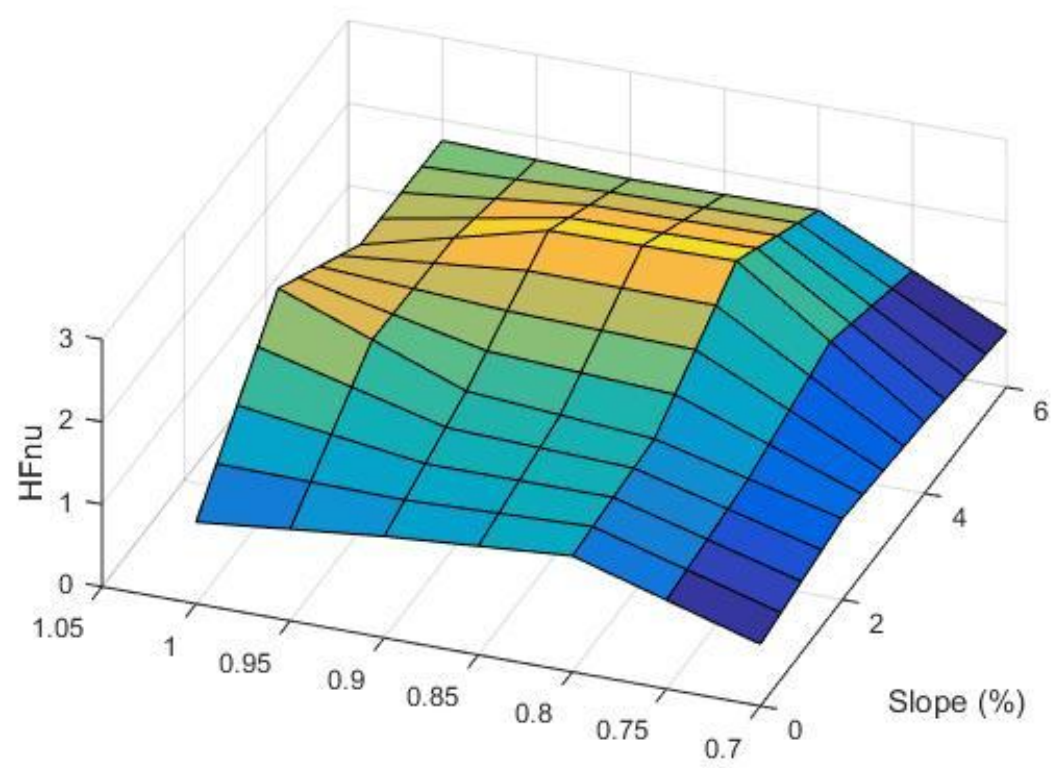

Speed (fraction of V)

Figure $16-\mathrm{HF}_{\mathrm{nu}}$ for each combination of speed and incline.

HFnu $=-0.09+0.28 \cdot$ speed $+0.008 \cdot$ speed $\cdot$ slope 
Table 23 - Statistical details for equation 25.

\begin{tabular}{lccc} 
& error & p-value & \\
\hline intercept & 0.10 & 0.38 & \\
speed coef. & 0.12 & 0.04 & \\
$\begin{array}{l}\text { interaction } \\
\text { coef. }\end{array}$ & 0.007 & 0.25 & \\
& & & \\
& p-value & adjusted r ${ }^{2}$ & $\mathrm{~F}$ \\
\hline model & 0.05 & 0.28 & 3.93 \\
\hline
\end{tabular}

$H F n u=-0.21+0.38 \cdot H R_{r e l}$

Table 24 - Statistical details for equation 26.

\begin{tabular}{lccc} 
& error & p-value & \\
\hline intercept & 0.12 & 0.08 & \\
$\mathrm{HR}_{\text {rel }}$ coef. & 0.12 & 0.005 & \\
& & & \\
& p-value & adjusted $r^{2}$ & $\mathrm{~F}$ \\
\hline model & 0.005 & 0.40 & 10.95 \\
\hline
\end{tabular}

We compared the 2 models, equations 25 and 26, and present the results in Table 25. As the simplest model $\left(\mathrm{HF}_{\mathrm{nu}}\right.$ as a function of $\left.\mathrm{HR}_{\mathrm{rel}}\right)$ also has the smaller RSS, we considered it to be better suited to explain our data. 
Table 25 - Statistical details for the comparison between models.

\begin{tabular}{lrr}
\hline $\begin{array}{l}\text { Independent } \\
\text { variable }\end{array}$ & $\mathrm{DF}$ & $\mathrm{RSS}$ \\
\hline $\mathrm{v}+\mathrm{v} \cdot \mathrm{i}$ & 13 & 0.038 \\
$\mathrm{HR}_{\mathrm{rel}}$ & 14 & 0.034 \\
& $\mathrm{p}$-value & $\mathrm{F}$ \\
\hline Comparison & - & - \\
\hline DF degrees of freedom; RSS & residual \\
sum of squares. &
\end{tabular}

4.2.2.3 LF:HF ratio

In Table 26 we present the LF:HF ratio values obtained in Experiment 2.

Table 26 - LF:HF ratio in Experiment 2 presented as mean (s.d.).

\begin{tabular}{|c|c|c|c|c|}
\hline \multirow{2}{*}{$\begin{array}{c}\text { Speed } \\
\text { (fraction } \\
\text { of } V \text { ) }\end{array}$} & \multicolumn{4}{|c|}{ Slope (\%) } \\
\hline & 0 & 2 & 4 & 6 \\
\hline \multirow[t]{2}{*}{0.7} & 2.45 & 2.31 & 2.02 & 3.13 \\
\hline & $(0.78)$ & $(1.69)$ & $(1.54)$ & $(0.69)$ \\
\hline \multirow[t]{2}{*}{0.8} & 1.68 & 0.99 & 0.51 & 1.16 \\
\hline & (3.77) & $(0.42)$ & $(0.92)$ & (1.55) \\
\hline \multirow[t]{2}{*}{0.9} & 1.64 & 0.60 & 0.57 & 1.00 \\
\hline & $(1.43)$ & $(0.91)$ & $(1.89)$ & $(2.19)$ \\
\hline \multirow[t]{2}{*}{1.0} & 1.68 & 0.45 & 0.55 & 0.80 \\
\hline & $(1.92)$ & $(0.96)$ & $(0.80)$ & (2.34) \\
\hline
\end{tabular}


We present the LF:HF values in Fig. 17. The multivariable model analysis resulted in equation 27 (associated statistics are presented in Table 27). A second model was fitted, with the ratio as a function of $\mathrm{HR}_{\text {rel }}$, regardless of the combination of speed and slope (equation 28, associated statistics are presented in Table 28).

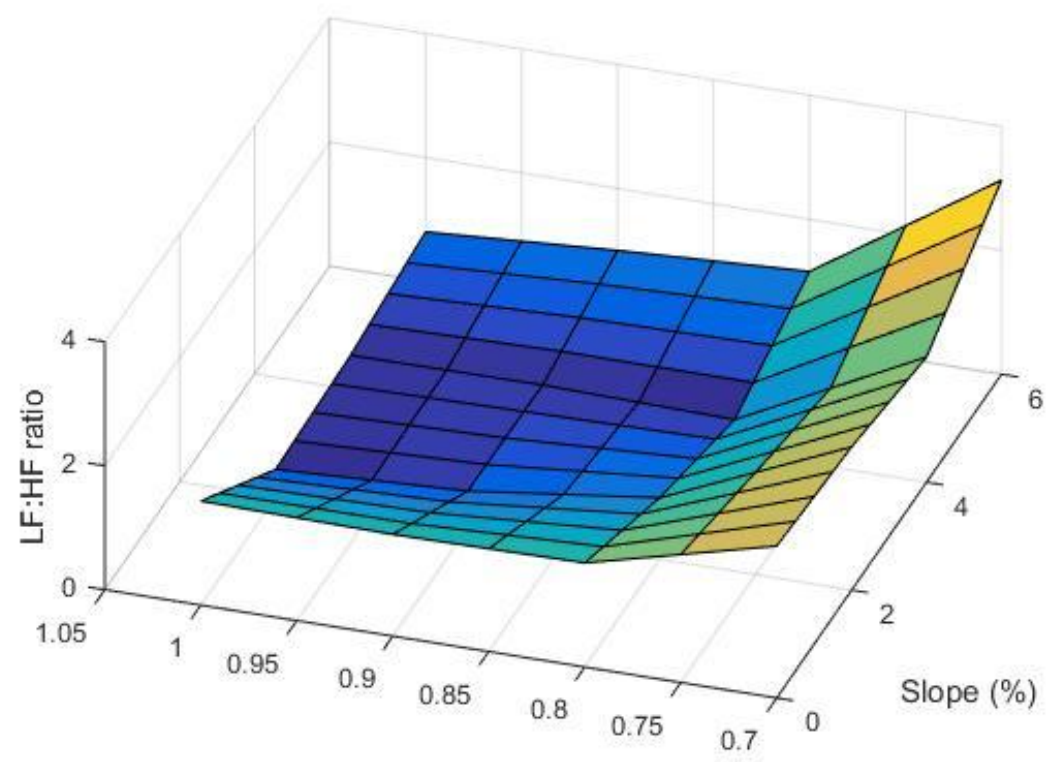

Speed (fraction of V)

Figure 17 - LF:HF ratio for each combination of speed and incline

$L F: H F=5.56-4.72 \cdot$ speed $-0.08 \cdot$ speed $\cdot$ slope

Table 27 - Statistical details for equation 27.

\begin{tabular}{lccc} 
& error & p-value & \\
\hline intercept & 1.15 & $<0.001$ & \\
speed coef. & 1.36 & 0.004 & \\
interaction & 0.08 & 0.32 & $\mathrm{~F}$ \\
coef. & & & \\
& $\mathrm{p}-\mathrm{value}$ & adjusted $\mathrm{r}^{2}$ & \\
\hline model & 0.007 & 0.46 & 7.38 \\
\hline
\end{tabular}


$L F: H F=6.74-5.14 \cdot H R_{r e l}$

Table 28 - Statistical details for equation 28.

\begin{tabular}{lrcr} 
& error & p-value & \\
\hline intercept & 1.35 & $<0.001$ & \\
HR $_{\text {rel }}$ coef. & 1.35 & 0.001 & \\
& & & \\
& $p$-value & adjusted $r^{2}$ & F \\
\hline model & 0.001 & 0.50 & 16.19 \\
\hline
\end{tabular}

We compared the 2 models, equations 27 and 28, and present the results in Table 29. As the simplest model (LF:HF as a function of $H R_{\text {rel }}$ ) also has the smaller RSS, we considered it to be better suited to explain our data. Hence, LF:HF is best explained by $\mathrm{HR}_{\mathrm{rel}}$.

Table 29 - Statistical details for the comparison between models.

\begin{tabular}{lrr}
$\begin{array}{lrr}\text { Independent } \\
\text { variable }\end{array}$ & DF & RSS \\
\hline$v+v \cdot i$ & 13 & 4.66 \\
$H_{\text {rel }}$ & 14 & 4.62 \\
& p-value & F \\
\hline Comparison & - & \\
\hline DF degrees of freedom; RSS residual \\
sum of squares.
\end{tabular}




\subsubsection{Considerations regarding absolute power}

In our experiment, we observed a decrease in both LF and HF absolute values as $\mathrm{HR}_{\text {rel }}$ increases. A reduction in HRV total power, and therefore in each spectral component, with tachycardia has been extensively reported (GUIDELINES, 1996; BAILÓN et al., 2013; DONG, 2016), hence, our data are in accordance with what is reported in the literature and with our hypothesis. The reduction in total power during exercise has been attributed to vagal withdrawal, so much that the majority of such decrease, when comparing rest to exercise, takes place until about $25-30 \%$ of the $\mathrm{VO}_{2 \max }($ PERINI \& VEICSTEINAS, 2003).

As previously mentioned, even at the lowest intensity runs $(0.7 \mathrm{~V}$ speed, $0 \%$ incline), subjects attained a mean HR of 127 BPM, which corresponds to $\sim 68 \%$ of estimated maximum HR. At such exercise intensity, the influence of the parasympathetic nervous system is much likely restricted (CARTER et al., 2003; PERINI \& VEICSTEINAS, 2003). Therefore, the observed reduction in total power found in our experiment should be independent of the vagal withdrawn.

A decrease in variability (be it of heart rate variability, be it of blood pressure) has been associated with a lack of capacity of the cardiovascular system to properly respond to environmental (either external or internal) challenges in face of adequate autonomic tonus, as in disease or aging (CARTER et al., 2003). However, as pointed out by MALIK \& CAMM (1993), the decrease in spectral power, either total or in a given frequency band, will also occur if the sympathovagal tonus is either abolished or saturated. In such cases, the authors point out that the frequency bands of HRV cannot be taken as a proxy to autonomic tonus as usually done in resting conditions.

Using this logic under the context of high-intensity exercise, a reduction in total variability may indicate that the system, in this case, the cardiac output is in a condition of increased constraint as a result of needing to supply for higher metabolic demand. In other words, since our volunteers exercised at a high intensity, particularly upon reaching maximum (or close to maximum) heart rate, it is reasonable to say that the parasympathetic branch was suppressed and the sympathetic branch was leading towards saturation, therefore, not many alterations in such components would be feasible. This would result in a lower variability, and, at 
least regarding autonomic control, the system would have a reduced margin of flexibility to respond to environmental challenges while still supplying for the high metabolic demand.

\subsubsection{Considerations regarding normalized power and LF:HF ratio}

In our experiments $L_{\mathrm{nu}}$ power was inversely proportional to $H R_{\mathrm{rel}}$, as hypothesized. $\mathrm{HF}_{\mathrm{nu}}$ increased with $\mathrm{HR}_{\mathrm{rel}}$, also according to our expectations.

The study of SUMl et al. (2006), for example, analyzed the HRV power spectrum in 10 female runners during a $3 \mathrm{~km}$ time trial, therefore, at high exercise intensity. We briefly reanalyze data from the aforementioned paper in order to include normalized values and exclude data obtained from rest and during the first lap, as HR was not compatible with the range encompassed in our experiments and the rest-exercise transition is not in the scope of the present study. We fitted linear models to heart rate per lap and the remaining variables as a function of heart rate. Considering this data subset, there is an increase in HR as exercise progresses $(p$-value $=0.005)$ and a decrease in total power $(p$-value $=0.007)$, absolute LF $(p$-value < 0.001) and HF power ( $p$-value $=0.005)$, similarly to our results. No changes in normalized LF power were found ( $p$-value $=0.69$ ). Normalized HF power showed a tendency to increase with $H R$, resulting in a decreasing LF:HF ratio, although none reached significance level (respectively, $p$-value $=0.08$ and 0.10 ).

In general, studies report that during high-intensity exercise, increments in exercise intensity leads to normalized LF power to either decrease (PERINI et al., 1990; PERINI \& VEICSTEINAS, 2003; BAILÓN et al., 2013) or remain unchanged (SUMI et al., 2006, reanalyzed).

As parasympathetic activity is notably reduced at the exercise intensity range included in the present project, increments in HR are met by increase in sympathetic tonus and/or hormonal adjustments.

The importance of increased sympathetic activity at moderate-high exercise intensity is well known. One could hypothesize that the inversely proportional relationship between $\mathrm{HR}_{\text {rel }}$ and $\mathrm{LF}_{\text {nu }}$ indicates that increments in sympathetic tonus 
are not being observed in the LF band as LF peak has been reported to shift to frequencies below $0.04 \mathrm{~Hz}$, traditionally known as VLF band (PERINI \& VEICSTEINAS, 2003). This hypothesis is supported by an increased VLF power in the elderly, which was interpreted as being associated with the age-related increase in sympathetic tonus (PERINI \& VEICSTEINAS, 2003). For reasons already mentioned, VLF will not be discussed in our study. However, it should be pointed out that we did not observe an increase in VLF normalized with $\mathrm{HR}_{\text {rel }}$, actually, quite the opposite. Therefore, this hypothesis does not seem to properly explain our data.

Alternatively, the observed pattern of $L_{\mathrm{nu}}$ might indicate greater importance of hormonal mechanisms (CARTER et al., 2003) and even a replacement of part of sympathetic tonus by such (KAMATH et al., 1991 apud SUMI et al., 2006).

Epinephrine and norepinephrine increase with exercise intensity (MCARDLE et al., 2006). Catecholamines act upon the heart increasing contraction force and excitability, therefore, resulting in an increment in stroke volume. They also lead to an increment in heart rate due to increased SA discharge rate and increased AV nodal conduction velocity. Catecholamines also play a role in vascular resistance, altering blood pressure (HALL, 2011).

KINDERMANN (1982), for example, measured epinephrine and norepinephrine concentration following aerobic (50-minute run at the anaerobic threshold, mean HR = 176 BPM) and anaerobic activity (run at 156\% maximum exercise capacity, resulting in an average of 1.5 minutes of activity, with increasing $\mathrm{HR}$ up to $185 \mathrm{BPM}$ ) in 17 trained males (mean age = 23 years). Such exercise intensities are approximately comparable with the lowest and highest intensity runs adopted in our study, at least regarding HR. They report a 15 fold increase in adrenaline and noradrenaline through the course of the aerobic activity. The first measure was taken 15 minutes after the beginning of the exercise, but those hormones already seem to present an increase in concentration in comparison to rest values. During the anaerobic bout of activity, adrenaline increased 3-4 fold and noradrenaline 6-9 fold. This study shows that even short periods of exercise may result in increased venous concentrations of catecholamines, which, among other adjustments, may lead to increasing HR. 
It should be mentioned that a greater contribution of a hormonal component on HRV does not exclude the aspects raised by MALIK \& CAMM (1993) regarding the interpretation of the FFT spectral power.

In our experiments, $\mathrm{HF}_{\mathrm{nu}}$ presented a linear increase with $\mathrm{HR}_{\mathrm{rel}}$. Normalized HF is usually reported to increase (BLAIN et al. 2009; PERINI \& VEICSTEINAS, 2003; MICHAEL et al., 2017) or remain unchanged (PERINI et al., 1990) as exercise intensity increases under moderate-high intensity.

The upper limit of the HF was set at $0.8 \mathrm{~Hz}$ in order to allow such band to include the components expected to reflect breathing frequency (PERINI \& VEICSTEINAS, 2003; BAILÓN et al., 2013; ALIKHANI et al., 2017). We believe the increase in $\mathrm{HF}_{\mathrm{nu}}$ with exercise intensity observed in our study reflects the mechanical effect of hyperventilation, in accordance with PERINI \& VEICSTEINAS (2003) and BAILÓN et al. (2013). Due to technical limitations, we were unable to obtain accurate measures of ventilatory frequency. Still, considering the HR our volunteers attained, it is safe to say that exercise intensity was enough as to elicit high ventilatory frequency.

We observed an inversely proportional relationship between LF:HF ratio and $\mathrm{HR}_{\mathrm{rel}}$. Other studies reported a decrease in LF:HF ratio with exercise intensity, but most do so by comparing conditions of lower metabolic demand than the ones here analyzed, such as resting condition (PERINI et al., 1990; SUMI et al., 2006). Both these studies found LF:HF to be lower during exercise than at rest, but unchanged throughout the exercise, despite the different intensities adopted (incremental protocol and time trial). The decrease in LF:HF ratio reflects both the decrease in $\mathrm{LF}_{\mathrm{nu}}$ and the increase in $\mathrm{HF}_{\mathrm{nu}}$, as previously discussed.

\subsubsection{6 $\mathrm{C}$ band $(1.3-1.6 \mathrm{~Hz})$}

We present the absolute and normalized power in C band in Table 30. In Fig. 18 , we present the power of $C_{n u}$ band for different combinations of speed and slope. The multivariable model analysis resulted in equation 29 (associated statistics are presented in Table 31). A second model was fitted, with $C_{n u}$ as function of $H R_{r e l}$, 
regardless of the combination of speed and slope (equation 30, associated statistics are presented in Table 32).

Table 30 - Mean absolute $\left(\mathrm{s}^{2} / \mathrm{Hz}\right)$ and normalized power in $\mathrm{C}$ band in Experiment 2 presented as mean (s.d.).

\begin{tabular}{|c|c|c|c|c|c|c|c|c|}
\hline \multirow{3}{*}{$\begin{array}{c}\text { Speed } \\
\text { (fraction } \\
\text { of } \mathrm{V} \text { ) }\end{array}$} & \multicolumn{8}{|c|}{ Slope (\%) } \\
\hline & \multicolumn{4}{|c|}{ Absolute $\left(\cdot 10^{-7}\right)$} & \multicolumn{4}{|c|}{ Normalized } \\
\hline & 0 & 2 & 4 & 6 & 0 & 2 & 4 & 6 \\
\hline \multirow[t]{2}{*}{0.7} & $0(0)$ & $0(0)$ & 0.30 & 1.60 & $0(0)$ & $0(0)$ & 0.001 & 0.001 \\
\hline & & & $(0.74)$ & (3.91) & & & $(0.001)$ & $(0.002)$ \\
\hline \multirow[t]{2}{*}{0.8} & $0(0)$ & 3.17 & 3.17 & 3.35 & $0(0)$ & 0.011 & 0.035 & 0.021 \\
\hline & & $(6.12)$ & $(3.80)$ & $(4.63)$ & & $(0.018)$ & $(0.044)$ & $(0.028)$ \\
\hline \multirow[t]{2}{*}{0.9} & 3.69 & 7.98 & 8.16 & 6.09 & 0.013 & 0.046 & 0.081 & 0.070 \\
\hline & (8.51) & $(11.25)$ & $(5.77)$ & $(3.86)$ & $(0.030)$ & $(0.048)$ & $(0.058)$ & $(0.044)$ \\
\hline \multirow[t]{2}{*}{1} & 5.10 & 12.27 & 16.01 & 9.94 & 0.020 & 0.089 & 0.115 & 0.117 \\
\hline & (7.85) & $(19.00)$ & (10.96) & $(4.54)$ & $(0.030)$ & $(0.112))$ & $(0.085)$ & $(0.050)$ \\
\hline
\end{tabular}

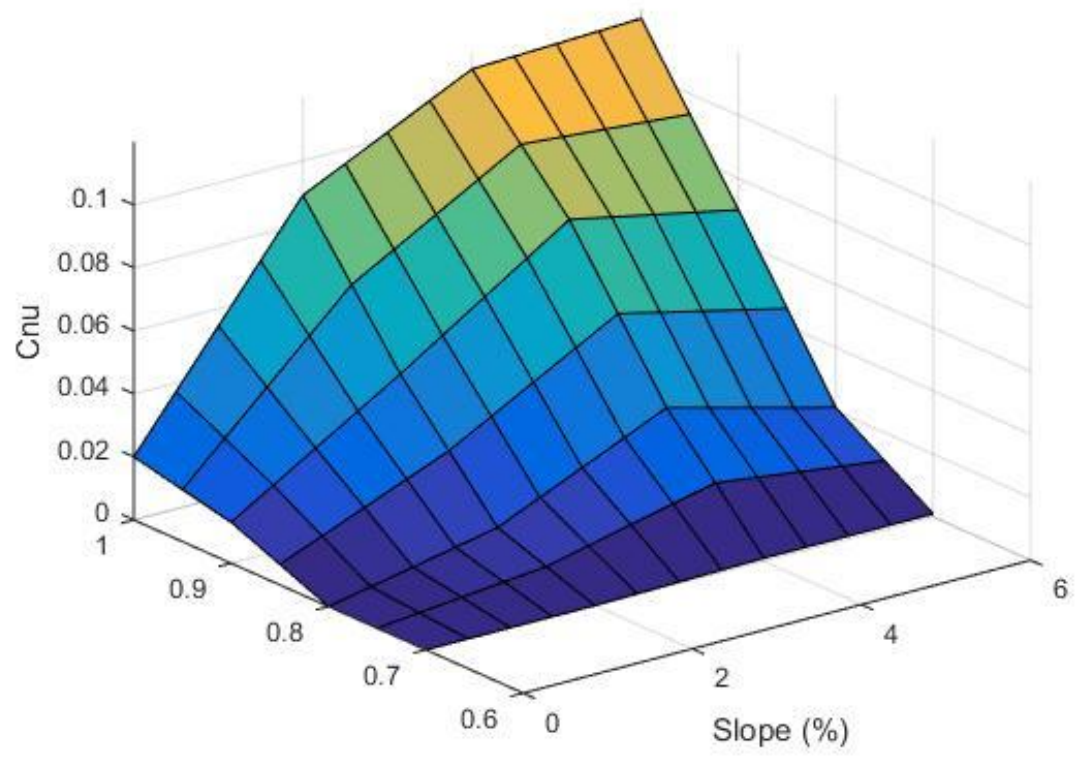

Speed (fraction of V)

Figure $18-C_{n u}$ for each combination of speed and incline. 
Cnu $=-0.21+0.26 \cdot$ speed $+0.01 \cdot$ speed $\cdot$ slope

Table 31 - Statistical details for equation 29.

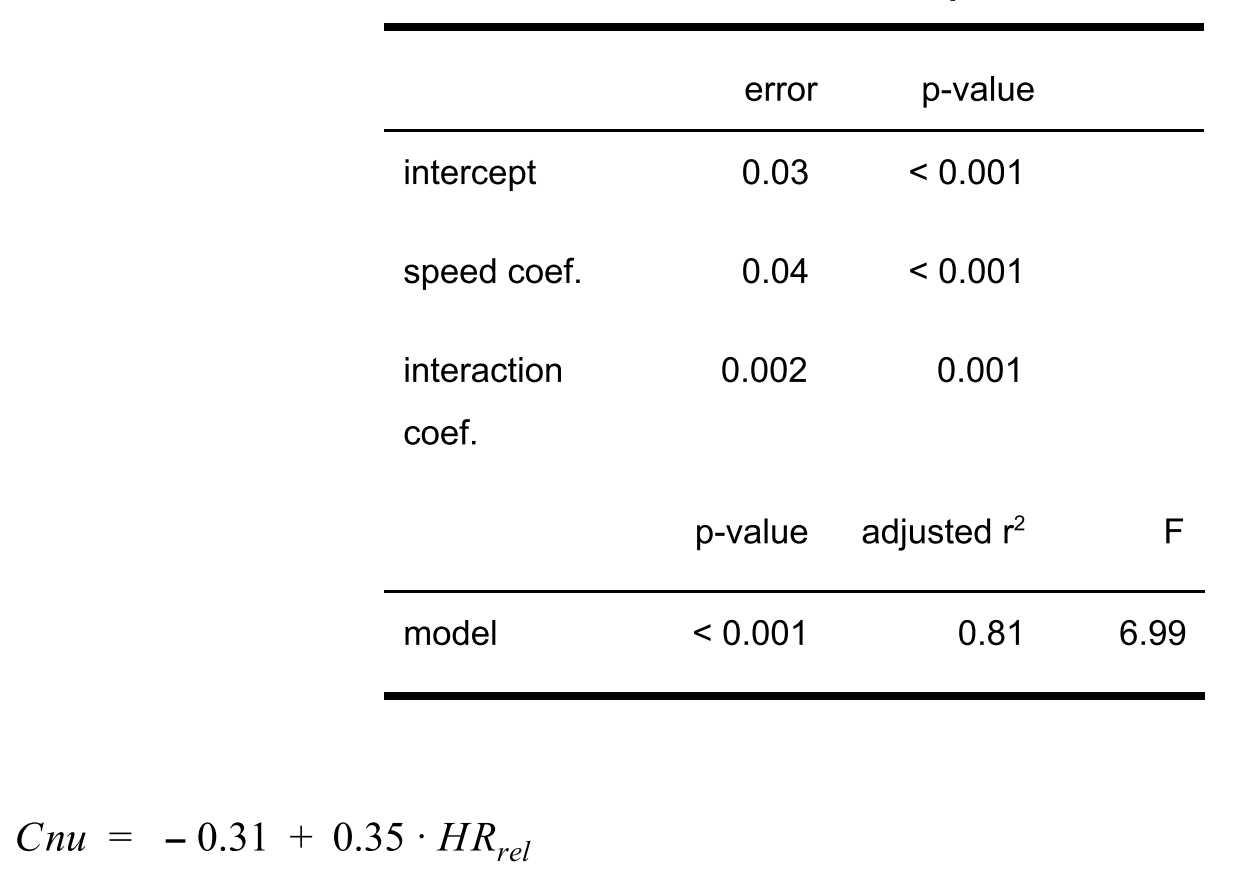

Table 32 - Statistical details for equation 30.

\begin{tabular}{lrrr} 
& error & p-value & \\
\hline intercept & 0.04 & 0.001 & \\
$\mathrm{HR}_{\text {rel }}$ coef. & 0.04 & $<0.001$ & \\
& & & \\
& p-value & adjusted $r^{2}$ & $\mathrm{~F}$ \\
\hline model & $<0.001$ & 0.81 & 64.86 \\
\hline
\end{tabular}

We compared the 2 models, equations 29 and 30, and present the results in Table 33. As both models equally explain $C_{n u}$, we selected the simplest model, with only $\mathrm{HR}_{\mathrm{rel}}$ as the predictor variable. 


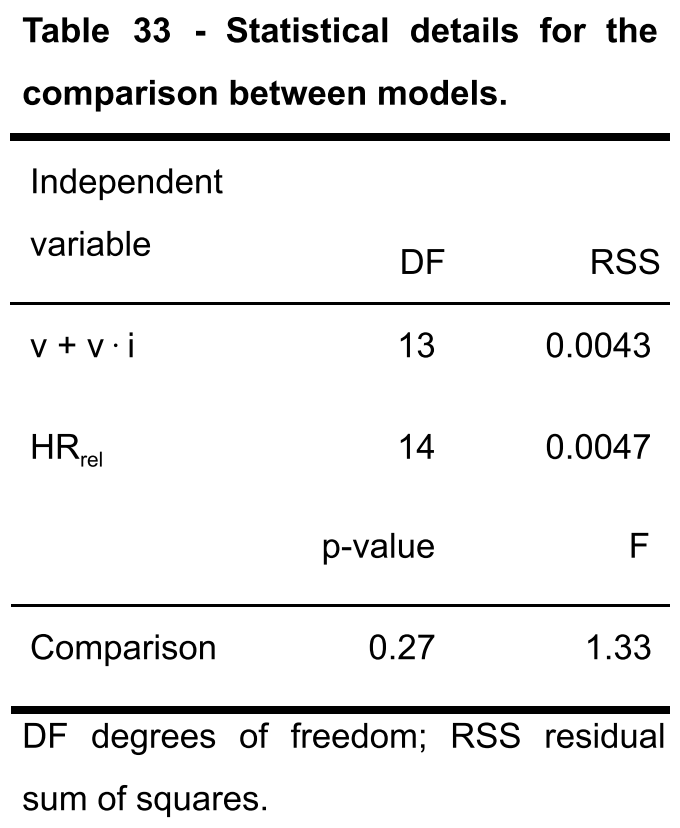

As expected, we observed a non-neglectable, although small, power at a frequency corresponding to that of cadence $\left(\mathrm{C}_{\mathrm{nu}}\right.$, from 1.3 to $\left.1.6 \mathrm{~Hz}\right)$. More precisely, this frequency band corresponds to half of the observed cadence.

Our results agree with those reported by BLAIN et al. (2009), in the sense that power in the cadence component presented a remarkable increase as workload approached its maximum. On the other hand, some studies failed to observe increased power in frequency components associated with cadence as exercise intensity increases (BAILÓN et al., 2013). One important difference between the study of BAILÓN et al. (2013) and ours is that in the former, power associated with stride frequency was computed as not only that of cadence band (like in our study) but also that included in the sinus arrhythmia band.

ALIKHANI et al. (2017) found that the main cadiolocomotor component affecting HRV is cadence itself. In our experiment cadence presented a relatively small range and moreover, since the spectrum power was computed encompassing this entire range, eventual changes in peak frequency with speed and slope (when $\mathrm{C}_{\mathrm{nu}}$ is present) would have been masked. More likely, our results are less associated with stride frequency itself but reflect increased power in $C_{n u}$ due to increased muscular contraction force, either propelling one further as with increasing stride length during increments in speed or resultant of additional work to increase the 
body's center of mass. This interpretation explains why the model of $\mathrm{C}_{\mathrm{nu}}$ as a function of $\mathrm{HR}_{\mathrm{rel}}$ was the most appropriate one.

Our results further support the hypothesis of a mechanical origin for the cadence component in HRV. As previously mentioned, the venous return would increase during muscle contraction and decrease during the relaxation phase. Such modulation is dependent on both cadence frequency and workload (BLAIN et al., 2009).

Still, the possible role of muscular metaboreceptors (FISHER, 2014, see "Introduction") influencing HRV on our experiments should not be overlooked. On the other hand, the importance of mechanoreceptors should be more restricted. Since we worked at a high-intensity exercise range, we expect the role of the sympathetic branch to have a bigger influence than that of parasympathetic tone. For this reason, we believe mechanoreceptors in skeletal muscle had a lower impact on HRV.

\subsubsection{In conclusion...}

In Table 34 we present a summary of the results obtained for the frequency domain HRV estimators.

It is interesting to note that despite the disparity in muscle activation pattern when running faster or uphill (SLONIGER et al., 1997), most of the HRV estimators here studied had their change pattern best explained by relative HR. Of course, such conclusion does not mean that peripheral components associated with different combinations of speed and slope that result in the same HR do not play a role in HRV and autonomic control of HR, so much that one of the estimators (a1ApEn) indeed responded differently to running conditions. It means, instead, that the component associated with metabolic demand is of greater importance. Some possible relevant components, as previously discussed, are changes in hormone concentration, increased sympathetic tonus, increased breathing frequency and increased skeletal muscle contraction force.

The heart is responsible for introducing enthalpy in the form of pressure into the system. Heart rate usually responds to global changes, such as alterations in 
blood pressure, venous return, and $\mathrm{CO}_{2}$ concentration. Thus, it makes sense that, at least regarding the changes of autonomic origin in HRV, the metabolic demand is the most important factor in heart rate control. Mechanical factors, associated with a greater venous return during respiration, speed or muscle contraction, also contributed to HRV.

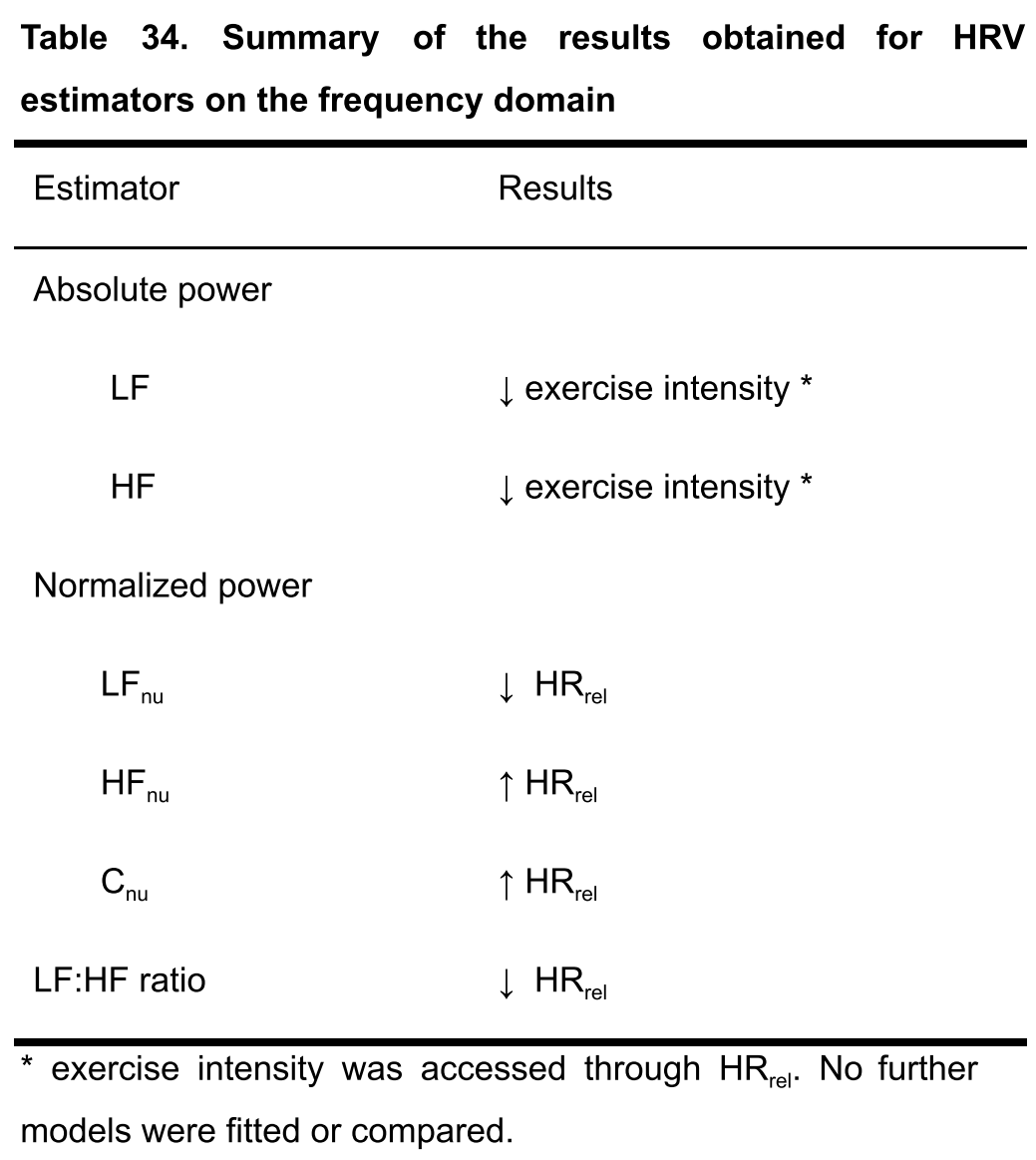

\subsection{QUESTION 3}

In Figs. 19 and 20 we bring attention to the age and $V$ speed distribution, respectively, amongst the populations from Experiments 1 and 2. Such data are also presented in Tables 2 and 3.

As can be observed, Experiment 1 presents a broader range of both age and fitness level when compared to Experiment 2. Results from the t-test indicate that there is no difference in average velocity $V$ between groups ( $p$-value $=0.35$ ), but 
volunteers from Experiment 1 are significantly older than those from Experiment 2 $(p$-value $=0.05)$.

As described in "Materials and Methods", HRV estimators for the experimental conditions of Experiment 1 were predicted according to the models obtained from the population in Experiment 2 and compared with collected data.

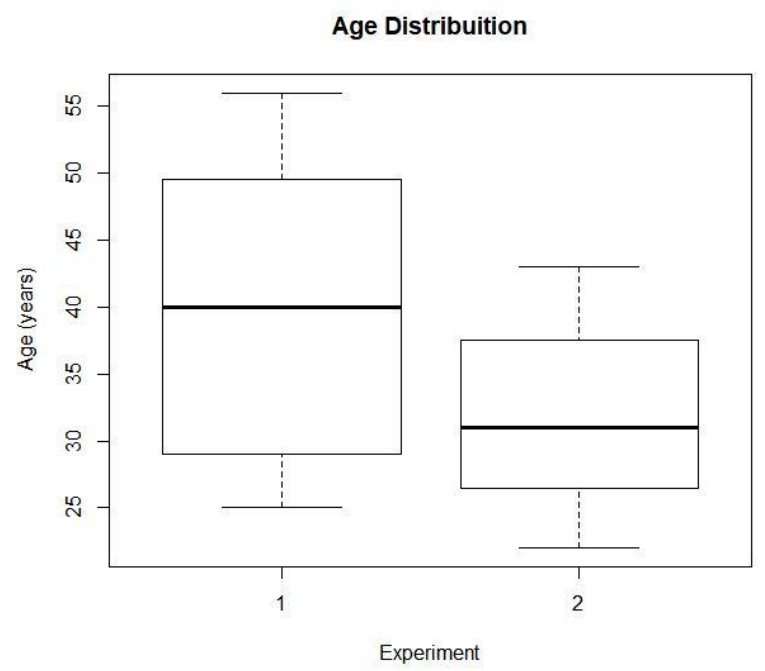

Figure 19 - Age distribution in population in

Experiments 1 and 2

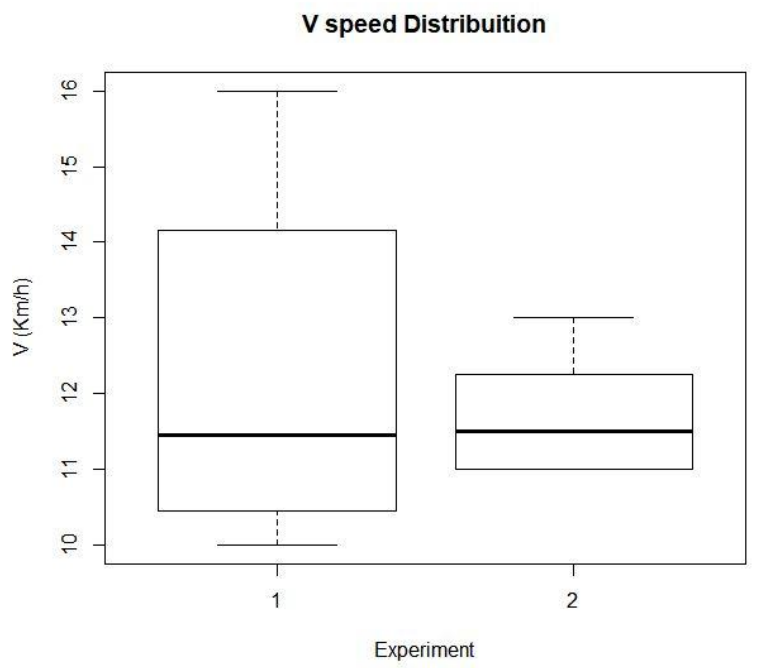

Figure 20 - V speed distribution in population in Experiments 1 and 2. 


\subsubsection{Time domain estimators}

\subsubsection{Heart Rate}

In Table 35 we bring absolute and relative HR obtained in Experiment 1.

Table 35 - Absolute (Hz) and relative HR in Experiment 1, presented as mean (s.d).

\begin{tabular}{cccccc}
\hline & \multicolumn{5}{c}{ Slope $(\%)$} \\
Speed \\
(fraction \\
of V)
\end{tabular}

The comparison between experimental and predicted values indicate that the model used to predict $\mathrm{HR}_{\text {rel }}$ in Experiment 2 is not adequate for data from Experiment $1(t=-5.06, D F=8, p$-value $<0.001)$. The ratios between estimated and observed values are shown in Fig. 21. 


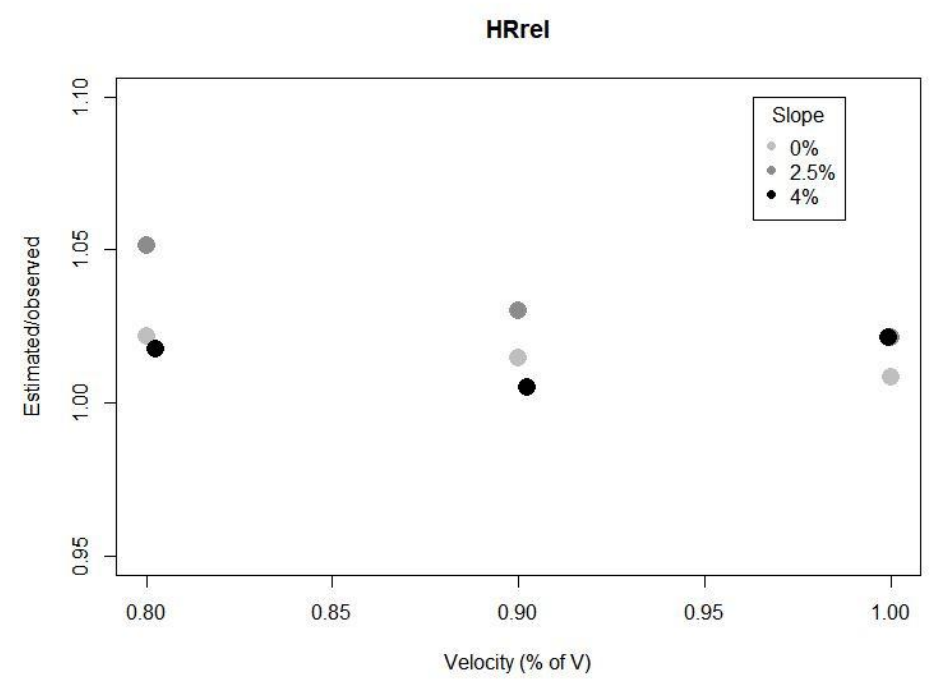

Figure 21 - Ratios between estimated and observed $H R_{\text {rel }}$ for different combinations of speed and slope.

We find that the model overestimates $H R_{\text {rel }}$ for the population in Experiment 1. A multivariable model was fitted to the estimated/observed $H R_{\text {rel }}$ ratio with speed and speed -slope as independent variables. The results show that the amount that is overestimated is not dependent on speed or the interaction speed $\cdot$ slope $(F=0.70$, adjusted $r^{2}=-0.08, p$-value $=0.53$ ).

A multivariable model with speed and slope as independent variables was fitted to $\mathrm{HR}_{\text {rel }}$ data obtained from Experiment 1 (equation 31). Statistics of the model are shown in Tables 36.

As expected, even though the model from Experiment 2 fails to explain data from Experiment 1, both models obtained present some similarities, that is, they are significant and the speed and interaction coefficients are positive. This result is rather obvious, but shows that speed and slope have the same general influence in $H R_{\text {rel }}$, but with different intensities for each population.

$$
H R_{\text {rel }}=0.30+0.70 \cdot \text { speed }+0.032 \cdot \text { speed } \cdot \text { slope }
$$


Table 36 - Statistical details for equation 31.

\begin{tabular}{|c|c|c|}
\hline \multicolumn{3}{|c|}{ Speed and slope } \\
\hline & error & $p$-value \\
\hline intercept & 0.05 & $<0.001$ \\
\hline speed coef. & 0.05 & $<0.001$ \\
\hline interaction coef. & 0.003 & $<0.001$ \\
\hline$p$-value & adjusted $r^{2}$ & $\mathrm{~F}$ \\
\hline$<0.001$ & 0.97 & 161.2 \\
\hline
\end{tabular}

The model from Experiment 2, as can be seen in Fig. 21, tends to overestimate $\mathrm{HR}_{\text {rel }}$ for the population that engaged in Experiment 1. This might be explained by the fact that the population which participated in Experiment 1 was, on average, older than those in Experiment 2. The capacity of the heart to respond to both sympathetic and vagal stimulation decreases with aging (LUCY et al., 2003; JAKOVLJEVIC, 2017), causing that, for a given relative exercise intensity (for example, a fraction of the $\mathrm{VO}_{2 \max }$ ), older individuals present a lower relative heart rate when compared to younger subjects (CARTER et al., 2003). While at lower exercise intensities the reduced HR can be explained by attenuated withdrawn of vagal stimulation, at higher intensities the reduction in sensitivity to sympathetic stimulation plays a more important role. TULPPO et al. (1998), for example, reported lower HR in older, compared to younger subjects, matched for fitness level, under submaximal and maximal intensities during incremental cycling protocol.

The reduced response to sympathetic stimuli also leads to an age-related decrease in maximum heart rate values (CARTER et al., 2003). Actually, $\mathrm{HR}_{\max }$ may decrease by $30 \%$ between 20 and 85 years of age (JAKOVLJEVIC, 2017). This is the main factor, regarding the cardiovascular system, responsible for the reduced maximum aerobic capacity in elderly. The decline in aerobic capacity is in the order of 3 - $6 \%$ per decade between 30 and 40 years, but increases to $20 \%$ per decade at age 70 (CARTER et al., 2003; JAKOVLJEVIC, 2017). Despite a reduced left ventricular ejection fraction and contraction force, during high-intensity exercise 
stroke volume is preserved. This is due to the fact that end diastolic volume is increased while end systolic volume is decreased in the elderly. Additionally, there is an increase in the contribution of the Frank-Starling mechanism in older individuals (JAKOVLJEVIC, 2017).

As cardiac output is lower in older individuals compared to younger ones at the same exercise intensity, in order to maintain a given arterial pressure, the former must increase vascular resistance (CARTER et al., 2003). This is accomplished by higher concentrations of noradrenaline (CARTER et al., 2003; JAKOVLJEVIC, 2017).

\subsubsection{The standard deviation of RR intervals (sdnn)}

In Table 37 we bring absolute and relative sdnn obtained in Experiment 1.

Table 37 - Absolute $\left(s \cdot 10^{-2}\right)$ and relative sdnn in Experiment 1, presented as mean (s.d).

\begin{tabular}{|c|c|c|c|c|c|c|}
\hline \multirow{3}{*}{$\begin{array}{l}\text { Speed } \\
\text { (fraction } \\
\text { of V) }\end{array}$} & \multicolumn{6}{|c|}{ Slope (\%) } \\
\hline & \multicolumn{3}{|c|}{ Absolute } & \multicolumn{3}{|c|}{ Relative } \\
\hline & 0 & 2.5 & 4 & 0 & 2.5 & 4 \\
\hline \multirow[t]{2}{*}{0.8} & 0.76 & 0.54 & 0.50 & 1.86 & 1.40 & 1.20 \\
\hline & $(0.04)$ & $(0.02)$ & $(0.04)$ & $(0.58)$ & $(0.46)$ & $(0.46)$ \\
\hline \multirow[t]{2}{*}{0.9} & 0.53 & 0.39 & 0.34 & 1.37 & 1.03 & 0.87 \\
\hline & $(0.01)$ & $(0.01)$ & $(0.01)$ & $(0.39)$ & $(0.34)$ & $(0.24)$ \\
\hline \multirow[t]{2}{*}{1} & 0.42 & 0.39 & 0.23 & 1.00 & 0.85 & 0.61 \\
\hline & $(0.02)$ & $(0.04)$ & $(0.01)$ & $(0.00)$ & $(0.34)$ & $(0.17)$ \\
\hline
\end{tabular}

The comparison between experimental and predicted values indicates that model used to predict $\mathrm{sdnn}_{\text {rel }}$ in Experiment 2 is not adequate for data from Experiment $1(\mathrm{t}=-2.89, \mathrm{DF}=8, \mathrm{p}$-value $=0.02)$. The ratios between estimated and observed values are shown in Fig. 22. 


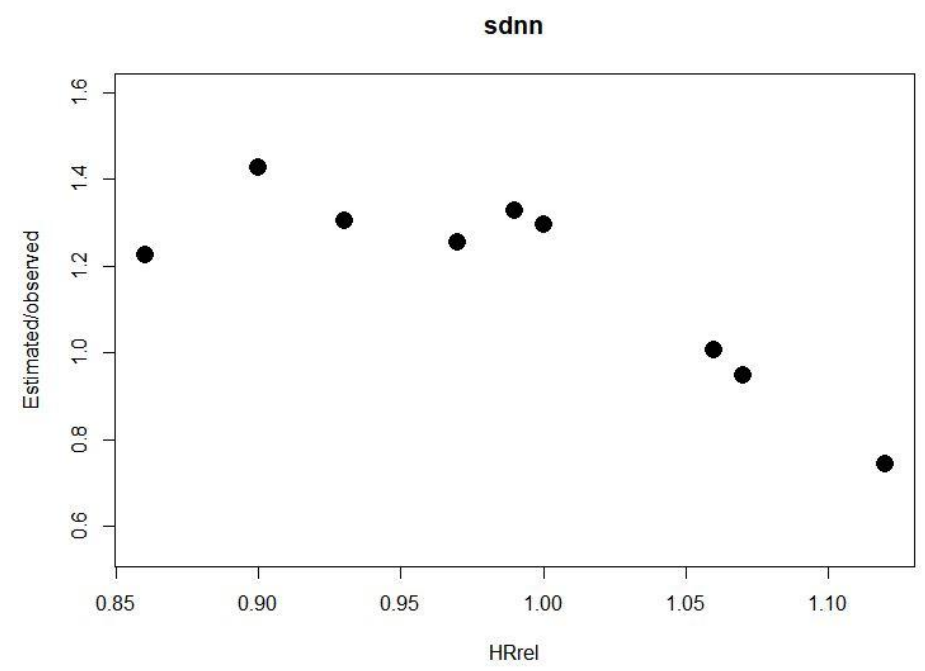

Figure 22 - Ratios between estimated and observed sdnn $_{\text {rel }}$ for different combinations of speed and slope.

We find that the model overestimates $\mathrm{sdnn}_{\text {rel }}$ for the population in Experiment 1 for lower $\mathrm{HR}_{\mathrm{rel}}$ and underestimates such variable under higher $\mathrm{HR}_{\mathrm{rel}}$. This pattern was confirmed by fitting a linear regression of the estimated/observed $s d n n_{\text {rel }}$ ratios per $\mathrm{HR}_{\text {rel }}\left(F=14.7\right.$, adjusted $r^{2}=0.63$, p-value $\left.=0.006\right)$.

A multivariable model with speed and slope as independent variables (equation 32) and a linear regression with $\mathrm{HR}_{\mathrm{rel}}$ as independent variable (equation 33) were fitted for $s d n n_{\text {rel }}$ data obtained from Experiment 1. Statistics of the models and results of their comparison are shown in Tables 38 and 39. Based on such results, we conclude the model of $s d n n_{\text {rel }}$ as function of $H R_{\text {rel }}$ best explains our data. sdnn $_{\text {rel }}=4.13-3.03 \cdot$ speed $-0.14 \cdot$ speed $\cdot$ slope $s d n n_{\text {rel }}=5.35-4.27 \cdot H R_{\text {rel }}$ 
Table 38 - Statistical details for equations 32 and 33.

\begin{tabular}{|c|c|c|c|c|c|c|}
\hline \multicolumn{4}{|c|}{$\mathrm{HR}_{\text {rel }}$} & \multicolumn{3}{|c|}{ Speed and slope } \\
\hline & & error & p-value & & error & p-value \\
\hline intercept & & 0.41 & $<0.001$ & intercept & 0.36 & $<0.001$ \\
\hline \multirow[t]{3}{*}{$\mathrm{HR}_{\mathrm{rel}}$ coef. } & & 0.41 & $<0.001$ & speed coef. & 0.40 & 0.002 \\
\hline & & & $<0.001$ & interaction coef. & 0.02 & 0.007 \\
\hline & $p$-value & adjusted $r^{2}$ & $\mathrm{~F}$ & $p$-value & adjusted $r^{2}$ & $\mathrm{~F}$ \\
\hline model & $<0.001$ & 0.92 & 107 & $<0.001$ & 0.93 & 54.61 \\
\hline
\end{tabular}

Table 39 - Statistical details for the comparison between models.

\begin{tabular}{lrr}
\hline $\begin{array}{l}\text { Independent } \\
\text { variable }\end{array}$ & DF & RSS \\
\hline $\mathrm{v}+\mathrm{v} \cdot \mathrm{i}$ & 6 & 0.057 \\
$\mathrm{HR}_{\mathrm{rel}}$ & 7 & 0.068 \\
& $\mathrm{p}$-value & $\mathrm{F}$ \\
\hline $\begin{array}{l}\text { Comparison } \\
\text { DF degrees of freedom; RSS residual }\end{array}$ \\
$\begin{array}{l}\text { sum of squares. } \\
\text { s.34 }\end{array}$
\end{tabular}

For both populations, from Experiments 2 and 1, the model that best explains $s d n n_{\text {rel }}$ is the one with $H R_{\text {rel }}$ as independent variable. In both cases, sdnn rel is inversely proportional to $H R_{\text {rel, }}$, but the impact of such variable on $s d n n_{\text {rel }}$ is of a bigger magnitude for the population studied on Experiment 2 than Experiment 1 (coefficients of -7.02 against -4.07 , respectively). This is further highlighted by the fact that the ratio between sdnn ${ }_{\text {rel }}$ values estimated using the model obtained in Experiment 2 and the observed $s d n n_{\text {rel }}$ in Experiment 1 is dependent on $\mathrm{HR}_{\text {rel }}$. For the lower intensities here studied, total variability is overestimated in Experiment 1, while it is underestimated at higher workloads. This shows a lower response of the sdnn 
estimator to experimental conditions. Other estimators, presented the same pattern (see results below).

The lower magnitude of change in $s d n n_{\text {rel }}$ in Experlment 1 compared to that observed in Experiment 2 and the overestimated/underestimated pattern observed might, again, be due to a higher relative exercise intensity performed by subjects in Experiment 1. As sdnn has been reported to decrease with exercise intensity in a curvilinear way (PERINI \& VEICSTEINAS, 2003; CARTER et al., 2003; MICHAEL et al., 2017), being at a higher relative intensity leads to a lower magnitude of change in sdnn with increasing metabolic demand. A second factor that might contribute to the observed pattern is an age-related lower responsiveness of cardiac control.

Still, some studies found no relationship between sdnn and age or fitness level. GREGOIRE et at. (1996) failed to observe any difference in sdnn (or any other estimator of $\mathrm{HRV}$, for that matter) when comparing middle-aged and young trained subjects cycling at 50 and $100 \mathrm{~W}$. CARTER et al. (2003) also found no reduction in total spectral power (numerically equivalent to sdnn) during steady-state exercise when comparing long-distance runners and sedentary subjects.

\subsubsection{Root mean square of successive differences of RR intervals (rms)}

In Table 40 we bring absolute and relative rms obtained in Experiment 1.

The comparison between experimental and predicted values indicates that the model used to predict $\mathrm{rms}_{\text {rel }}$ in Experiment 2 is not adequate for data from Experiment $1(\mathrm{t}=2.99, \mathrm{DF}=8, \mathrm{p}$-value $=0.02)$. The ratios between estimated and observed values are shown in Fig. 23. We find that the model underestimates $\mathrm{rms}_{\text {rel }}$ for the population in Experiment 1. A linear regression of the estimated/observed $\mathrm{rms}_{\mathrm{rel}}$ ratios per $\mathrm{HR}_{\text {rel }}$ was fitted. The results show that the amount that is underestimated is not dependent on $\mathrm{HR}_{\text {rel }}\left(F=0.27\right.$, adjusted $r^{2}=-0.09$, $p$-value $\left.=0.61\right)$ 
Table 40 - Absolute (ms) and relative rms for Experiment 1, presented as mean (s.d).

\begin{tabular}{ccccccc}
\hline & \multicolumn{5}{c}{ Slope (\%) } \\
$\begin{array}{c}\text { Speed } \\
\text { (fraction } \\
\text { of V) }\end{array}$ & \multicolumn{7}{c}{ Absolute } & & & Relative \\
\cline { 2 - 7 } & 0 & 2.5 & 4 & 0 & 2.5 & 4 \\
\hline 0.8 & 4.50 & 4.22 & 3.15 & 1.42 & 1.28 & 1.01 \\
& $(1.96)$ & $(2.15)$ & $(1.42)$ & $(0.51)$ & $(0.32)$ & $(0.42)$ \\
& 3.90 & 3.19 & 3.12 & 1.17 & 1.05 & 0.99 \\
& $(1.990)$ & $(1.55)$ & $(1.69)$ & $(0.23)$ & $(0.21)$ & $(0.51)$ \\
& 3.20 & 3.12 & 2.61 & 1.00 & 0.97 & 0.90 \\
& $(1.16)$ & $(1.24)$ & $(0.76)$ & $(0.00)$ & $(0.17)$ & $(0.21)$ \\
\hline
\end{tabular}

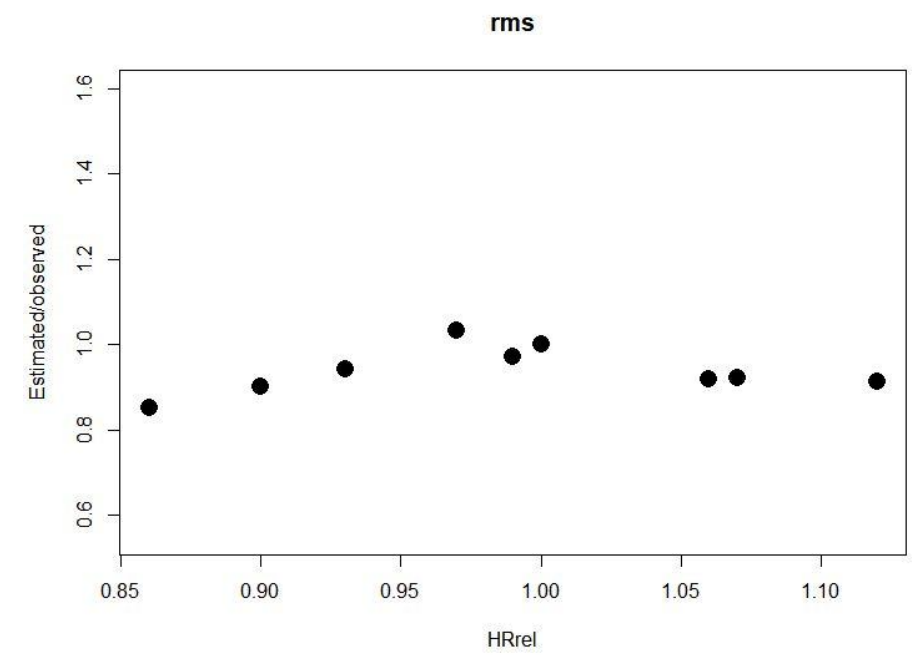

Figure 23 - Ratios between estimated and observed $\mathrm{rms}_{\mathrm{rel}}$ for different combinations of speed and slope.

A multivariable model with speed and slope as independent variables (equation 34) and a linear regression with $\mathrm{HR}_{\mathrm{rel}}$ as independent variable (equation 35) were fitted for $\mathrm{rms}_{\text {rel }}$ data obtained from Experiment 1 . Statistics of the models and results of their comparison are shown in Tables 41 and 42. Therefore, we conclude the model of $\mathrm{rms}_{\text {rel }}$ as function of $\mathrm{HR}_{\text {rel }}$ is the one that best explains our data. 


$$
\begin{aligned}
& r m s_{\text {rel }}=2.34-1.27 \cdot \text { speed }+-0.058 \cdot \text { speed } \cdot \text { slope } \\
& r m s_{\text {rel }}=2.90-1.83 \cdot H R_{\text {rel }}
\end{aligned}
$$

\begin{tabular}{|c|c|c|c|c|c|c|}
\hline \multicolumn{4}{|c|}{$\mathrm{HR}_{\text {rel }}$} & \multicolumn{3}{|c|}{ Speed and slope } \\
\hline & & error & p-value & & error & p-value \\
\hline intercept & & 0.29 & $<0.001$ & intercept & 0.31 & $<0.001$ \\
\hline \multirow[t]{3}{*}{$\mathrm{HR}_{\text {rel }}$ coef. } & & 0.29 & $<0.001$ & speed coef. & 0.34 & 0.01 \\
\hline & & & & interaction coef. & 0.02 & 0.02 \\
\hline & $p$-value & adjusted $r^{2}$ & $\mathrm{~F}$ & $p$-value & adjusted $\mathrm{r}^{2}$ & $\mathrm{~F}$ \\
\hline model & $<0.001$ & 0.82 & 39.69 & 0.006 & 0.75 & 13.24 \\
\hline
\end{tabular}

Table 41 - Statistical details for equations 34 and 35.

Table 42 - Statistical details for the comparison between models.

\begin{tabular}{lrr}
\hline $\begin{array}{l}\text { Independent } \\
\text { variable }\end{array}$ & DF & RSS \\
\hline $\mathrm{v}+\mathrm{v} \cdot \mathrm{i}$ & 6 & 0.041 \\
$\mathrm{HR}_{\mathrm{rel}}$ & 7 & 0.033 \\
& $\mathrm{p}$-value & $\mathrm{F}$ \\
\hline Comparison & - & - \\
\hline $\begin{array}{l}\text { DF degrees of freedom; RSS residual sum } \\
\text { of squares. }\end{array}$
\end{tabular}

As with sdnn $n_{\text {rel, }}$, for both populations from Experiments 2 and 1, the model that best explains $\mathrm{rms}_{\mathrm{rel}}$ is the one with $\mathrm{HR}_{\mathrm{rel}}$ as the independent variable. In both experiments, $\mathrm{rms}_{\mathrm{rel}}$ is inversely proportional to $\mathrm{HR}_{\mathrm{rel}}$. Although, we observe that the model from Experiment 2 underestimates $\mathrm{rms}_{\mathrm{rel}}$ data from Experiment 1. 
Such underestimation of rms was unexpected, since, as previously mentioned, subjects from Experiment 1 were probably at a higher relative exercise than those from Experiment 1, therefore, they should present similar or lower rms values. rms correlates with HF frequency band (TASKFORCE, 1996), and, as reported below, in our study HF was found to be similar in Experiment 1 and 2, much likely due to the effect of increased ventilation associated with exercise.This might be the reason why we found a higher than expected value of rms, and is in accordance with a slight increase in rms observed at high intensity exercise above 180 BPM (MICHAEL et al., 2017).

\subsubsection{Area 1 of Approximate Entropy (a1ApEn)}

In Table 43 we bring absolute and relative a1ApEn obtained in Experiment 1.

Table 43 - Absolute and relative a1ApEn in Experiment 1, presented as mean (s.d).

\begin{tabular}{|c|c|c|c|c|c|c|}
\hline \multirow{3}{*}{$\begin{array}{l}\text { Speed } \\
\text { (fraction } \\
\text { of } \mathrm{V} \text { ) }\end{array}$} & \multicolumn{6}{|c|}{ Slope (\%) } \\
\hline & \multicolumn{3}{|c|}{ Absolute } & \multicolumn{3}{|c|}{ Relative } \\
\hline & 0 & 2.5 & 4 & 0 & 2.5 & 4 \\
\hline \multirow[t]{2}{*}{0.8} & 0.23 & 0.26 & 0.25 & 0.82 & 0.96 & 0.89 \\
\hline & $(0.08)$ & $(0.06)$ & $(0.07)$ & $(0.19)$ & $(0.32)$ & $(0.22)$ \\
\hline \multirow[t]{2}{*}{0.9} & 0.27 & 0.28 & 0.28 & 0.97 & 1.01 & 1.02 \\
\hline & $(0.06)$ & $(0.07)$ & $(0.04)$ & $(0.20)$ & $(0.25)$ & $(0.25)$ \\
\hline \multirow[t]{2}{*}{1} & 0.28 & 0.30 & 0.30 & 1.00 & 1.05 & 1.10 \\
\hline & $(0.06)$ & $(0.07)$ & $(0.05)$ & $(0.00)$ & $(0.23)$ & $(0.27)$ \\
\hline
\end{tabular}

The comparison between experimental and predicted values indicates that the model used to predict $a 1 A p E n_{\text {rel }}$ in Experiment 2 is adequate for data from Experiment $1(\mathrm{t}=-1.18, \mathrm{DF}=8, \mathrm{p}$-value $=0.27)$. The ratios between estimated and observed values are shown in Fig. 24. 


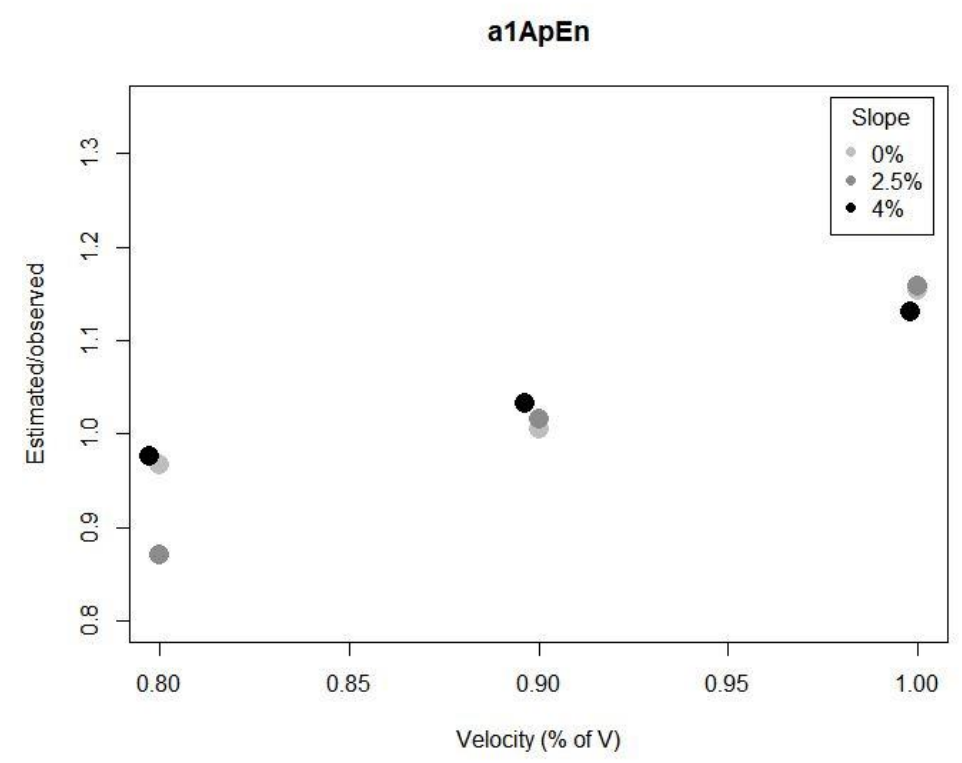

Figure 24 - Ratios between estimated and observed $\mathrm{a} 1 \mathrm{ApEn} \mathrm{n}_{\mathrm{rel}}$ for different combinations of speed and slope.

The fact that the model fitted for $\mathrm{a} 1 \mathrm{ApEn} \mathrm{n}_{\text {rel }}$ data collected from Experiment 2 is adequate to Experiment 1 despite possible complications associated with relative exercise intensity between both groups further supports the hypothesis that this estimator is sensitive to changes in HRV related with running conditions (speed and slope). More so, the range of $\mathrm{V}$ speed adopted for the population in Experiment 1 was broader than that used for Experiment 2 (respectively, from 10 to $16 \mathrm{~km} / \mathrm{h}$ and 11 to $13 \mathrm{~km} / \mathrm{h}$ ), indicating that a1ApEn responds to relative changes in speed, instead of absolute values. A relative-speed protocol had also been adopted by NATALI (2015), and the author observed results similar to ours.

\subsubsection{Frequency domain estimators}

4.3.2.1 LF band $(0.04-0.15 \mathrm{~Hz})$

In Table 43 we bring absolute and relative LF band obtained in Experiment 1. 
Table 43 - Absolute $\left(s^{2} / \mathrm{Hz}\right)$ and normalized power in LF band in Experiment 1 presented as mean (s.d.).

\begin{tabular}{|c|c|c|c|c|c|c|}
\hline \multirow{3}{*}{$\begin{array}{c}\text { Speed } \\
\text { (fraction } \\
\text { of } V \text { ) }\end{array}$} & \multicolumn{6}{|c|}{ Slope (\%) } \\
\hline & \multicolumn{3}{|c|}{ Absolute $\left(\cdot 10^{-5}\right)$} & \multicolumn{3}{|c|}{ Normalized } \\
\hline & 0 & 2.5 & 4 & 0 & 2.5 & 4 \\
\hline \multirow[t]{2}{*}{0.8} & 1.84 & 0.85 & 1.11 & 0.217 & 0.276 & 0.187 \\
\hline & $(3.46)$ & $(0.77)$ & $(2.98)$ & $(0.145)$ & $(0.184)$ & $(0.120)$ \\
\hline \multirow[t]{2}{*}{0.9} & 0.52 & 0.32 & 0.29 & 0.203 & 0.182 & 0.146 \\
\hline & $(0.42)$ & $(0.32)$ & $(0.45)$ & $(0.141)$ & $(0.133)$ & $(0.099)$ \\
\hline \multirow[t]{2}{*}{1} & 0.23 & 0.92 & 0.12 & 0.135 & 0.156 & 0.155 \\
\hline & $(0.19)$ & $(2.81)$ & $(0.13)$ & $(0.078)$ & $(0.115)$ & $(0.124)$ \\
\hline
\end{tabular}

\subsection{Absolute power}

The comparison between experimental and predicted values indicates that the model used to predict absolute LF in Experiment 2 is not adequate for data from Experiment $1(\mathrm{t}=-2.63, \mathrm{DF}=8, \mathrm{p}$-value $=0.03)$. The ratios between estimated and observed values are shown in Fig. 25. We find that the model overestimates absolute LF power for the population in Experiment 1. A linear model of the ratios estimated/observed absolute LF per $\mathrm{HR}_{\text {rel }}$ was fitted. The results show that the amount that is overestimated is not dependent on $\operatorname{HR}_{\text {rel }}\left(F=2.32\right.$, adjusted $r^{2}=0.14$, p-value $=0.17)$. 


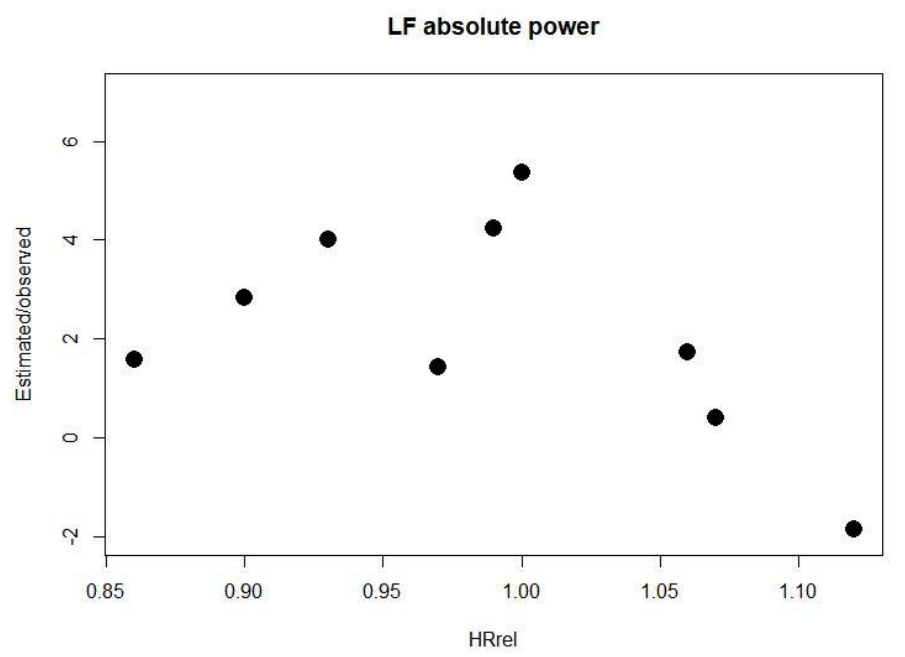

Figure 25 - Ratios between estimated and observed absolute LF power for different combinations of speed and slope.

In order to verify if absolute power decreased as exercise intensity increased in Experiment 1, we performed linear regression with $\mathrm{HR}_{\text {rel }}$ as the independent variable and absolute LF power as the dependent variable. As expected, like in Experiment 2, LF power decreased $(p$-value $=0.004)$ with increasing heart rate.

\subsection{Normalized power}

The comparison between experimental and predicted values indicates that the model used to predict $L F_{n u}$ in Experiment 2 is adequate for data from Experiment $1 \mathrm{t}$ $=0.40, \mathrm{DF}=8, \mathrm{p}$-value $=0.69)$. The ratios between estimated and observed values are shown in Fig. 26. A linear model of the ratios estimated/observed $L F_{n u}$ per $H R_{\text {rel }}$ was fitted. 


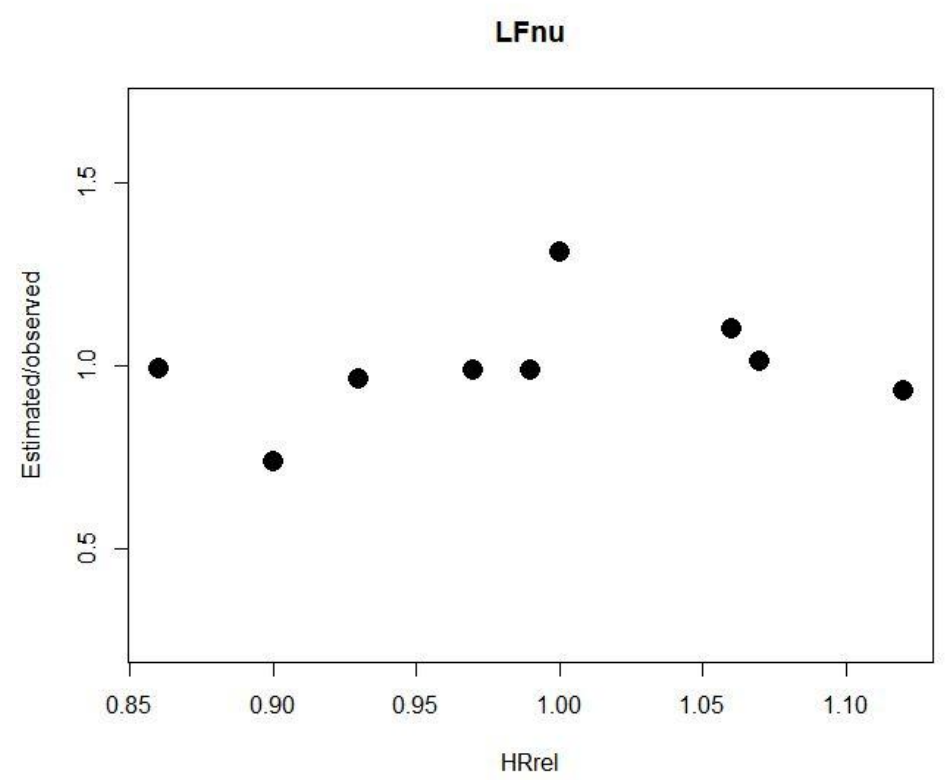

Figure 26 - Ratios between estimated and observed $L F_{n u}$ power for different combinations of speed and slope.

\subsubsection{2 $\mathrm{HF}$ band $(0.15-0.8 \mathrm{~Hz})$}

In Table 44 we bring absolute and relative HF band obtained in Experiment 1.

Table 44 - Absolute $\left(s^{2} / \mathrm{Hz}\right)$ and normalized power in $\mathrm{HF}$ band in Experiment 1 presented as mean (s.d.).

\section{Slope $(\%)$}

\begin{tabular}{ccccccc}
$\begin{array}{c}\text { Speed } \\
\text { (fraction } \\
\text { of } \mathrm{V})\end{array}$ & \multicolumn{3}{c}{ Absolute $\left(\cdot 10^{-5}\right)$} & \multicolumn{3}{c}{ Normalized } \\
\cline { 2 - 7 } & 0 & 2.5 & 4 & 0 & 2.5 & 4 \\
\hline 0.8 & 0.83 & 0.69 & 0.42 & 0.165 & 0.235 & 0.169 \\
& $(0.80)$ & $(0.53)$ & $(0.48)$ & $(0.101)$ & $(0.172)$ & $(0.111)$ \\
& & & & & & \\
0.9 & 0.52 & 0.44 & 0.36 & 0.178 & 0.283 & 0.245 \\
& $(0.44)$ & $(0.32)$ & $(0.38)$ & $(0.132)$ & $(0.260)$ & $(0.134)$ \\
& & & & & & \\
& 0.35 & 0.28 & 0.18 & 0.212 & 0.240 & 0.270 \\
& $(0.31)$ & $(0.18)$ & $(0.11)$ & $(0.183)$ & $(0.136)$ & $(0.154)$
\end{tabular}




\subsection{Absolute power}

The comparison between experimental and predicted values indicates that model used to predict $\mathrm{HF}$ in Experiment 2 is adequate for data from Experiment $1 \mathrm{t}=$ -1.70, DF $=8, p$-value $=0.13$ ). The ratios between estimated and observed values are shown in Fig. 27.

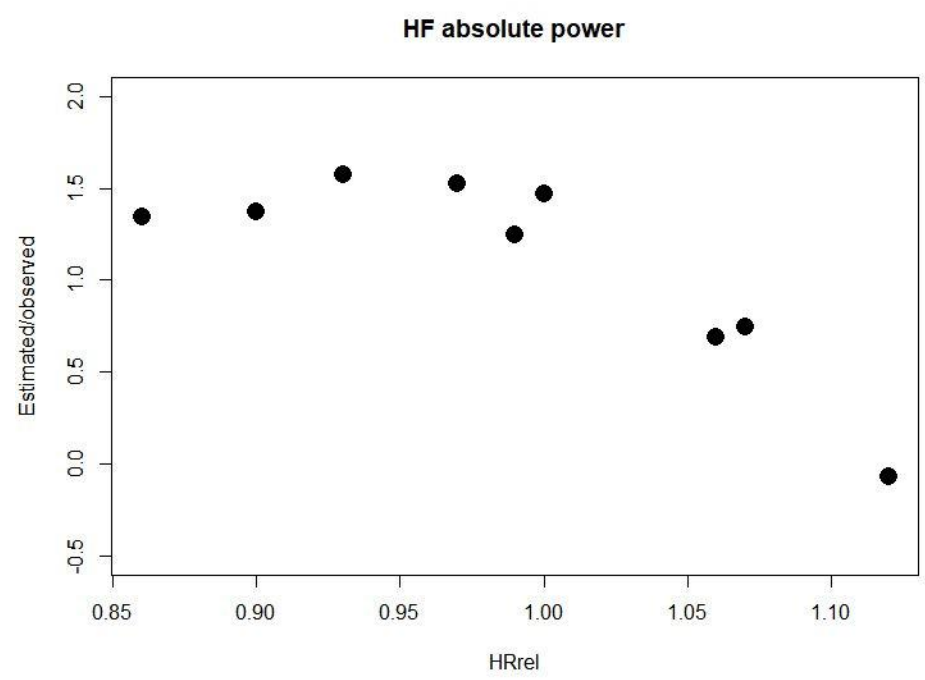

Figure 27 - Ratios between estimated and observed absolute HF power for different combinations of speed and slope.

\subsection{Normalized power}

The comparison between experimental and predicted values indicates that the model used to predict $\mathrm{HF}_{\mathrm{nu}}$ in Experiment 2 is not adequate for data from Experiment $1 \quad(t=5.55, D F=8, p$-value $<0.001)$. The ratios between estimated and observed values are shown in Fig. 28. We find that the model underestimates $\mathrm{HF}_{\mathrm{nu}}$ for the population in Experiment 1. A linear model of the ratio estimated/observed $\mathrm{HF}_{\mathrm{nu}}$ per $\mathrm{HR}_{\text {rel }}$ was fitted. The results show that the amount that is underestimated is not dependent on $\mathrm{HR}_{\mathrm{rel}} \quad\left(\mathrm{F}=1.39\right.$, adjusted $\mathrm{r}^{2}=0.05$, $\mathrm{p}$-value $\left.=0.28\right)$.

A multivariable model with speed and slope as independent variables (equation 36) and a linear regression with $\mathrm{HR}_{\mathrm{rel}}$ as the independent variable 
(equation 37) were fitted for $\mathrm{HF}_{\mathrm{nu}}$ data obtained from Experiment 1. Statistics of he models and results of their comparison are shown in Tables 45 and 46.

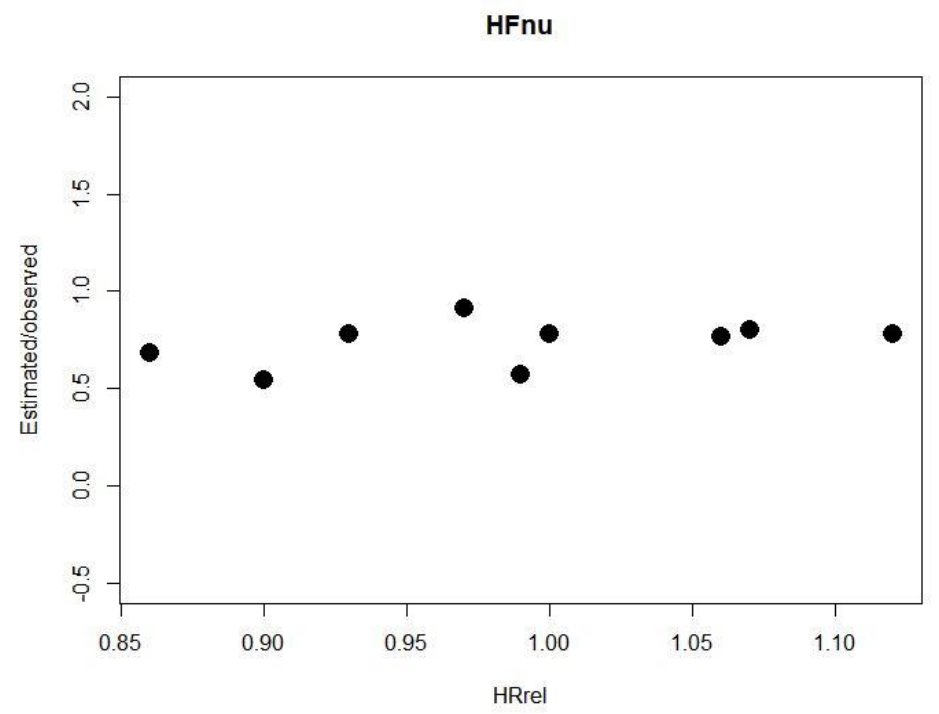

Figure 28 - Ratio between estimated and observed $\mathrm{HF}_{\mathrm{nu}}$ power for different combinations of speed and slope.

$H F n u=-0.007+0.224 \cdot$ speed $+0.014 \cdot$ speed $\cdot$ slope

$H F n u=-0.11+0.15 \cdot H R_{r e l}$

Table 45 - Statistical details for equations 36 and 37.

\begin{tabular}{|c|c|c|c|c|c|c|}
\hline \multicolumn{4}{|c|}{$H R_{\text {rel }}$} & \multicolumn{3}{|c|}{ Speed and slope } \\
\hline & & error & $\mathrm{p}$-value & & error & $p$-value \\
\hline intercept & & 0.15 & & intercept & 0.12 & 0.96 \\
\hline \multirow[t]{3}{*}{$\mathrm{HR}_{\text {rel }}$ coef. } & & 0.15 & & speed coef. & 0.14 & 0.17 \\
\hline & & & & interaction coef. & 0.008 & 0.12 \\
\hline & $p$-value & adjusted $r^{2}$ & $\mathrm{~F}$ & $p$-value & adjusted $r^{2}$ & $\mathrm{~F}$ \\
\hline model & 0.06 & 0.33 & 5.11 & 0.11 & 0.36 & 3.26 \\
\hline
\end{tabular}


Although it did not reach significance level, an increase in $\mathrm{HF}_{\mathrm{nu}}$ is observed with increasing $\mathrm{HR}_{\mathrm{rel}}$. This same pattern was reported for Experiment 2.

\subsubsection{LF:HF ratio}

In Table 47 we bring the LF:HF ratio obtained in Experiment 1.

Table 47. LF:HF ratio in Experiment 1.

\begin{tabular}{|c|c|c|c|}
\hline \multirow{2}{*}{$\begin{array}{l}\text { Speed } \\
\text { (fraction } \\
\text { of } \mathrm{V} \text { ) }\end{array}$} & \multicolumn{3}{|c|}{ Slope (\%) } \\
\hline & 0 & 2.5 & 4 \\
\hline 0.8 & $\begin{array}{c}1.32 \\
(3.20)\end{array}$ & $\begin{array}{c}1.18 \\
(3.82)\end{array}$ & $\begin{array}{c}1.11 \\
(1.68)\end{array}$ \\
\hline 0.9 & $\begin{array}{c}1.14 \\
(2.01)\end{array}$ & $\begin{array}{c}0.64 \\
(1.48)\end{array}$ & $\begin{array}{c}0.60 \\
(1.52)\end{array}$ \\
\hline 1 & $\begin{array}{c}0.63 \\
(1.56)\end{array}$ & $\begin{array}{c}0.65 \\
(4.12)\end{array}$ & $\begin{array}{r}0.56 \\
(1.06)\end{array}$ \\
\hline
\end{tabular}

The comparison between experimental and predicted values indicates that the model used to predict LF:HF ratio in Experiment 2 is not adequate for data from Experiment 1 ( $\mathrm{t}=-0.69$, DF $=8, \mathrm{p}$-value $<0.001)$. The ratios between estimated and observed values are shown in Fig. 29.

We find that the model overestimates LF:HF for the population in Experiment 1. A linear model of the ratio estimated/observed $L F: H F$ per $H_{\text {rel }}$ was fitted. The results show that the amount error between predicted and observed values is not dependent on $\mathrm{HR}_{\mathrm{rel}}\left(\mathrm{F}=0.24\right.$, adjusted $\mathrm{r}^{2}=-0.10$, p-value $\left.=0.63\right)$.

A multivariable model with speed and slope as independent variables (equation 38) and a linear regression with $\mathrm{HR}_{\mathrm{rel}}$ as the independent variable (equation 39) were fitted for LF:HF ratio data obtained from Experiment 1 . Statistics of the 
models and results of their comparison are shown in Tables 48 and 49. Based on such results, we conclude the model of LF:HF ratio as a function of $H R_{\text {rel }}$ is the one that best explains our data.

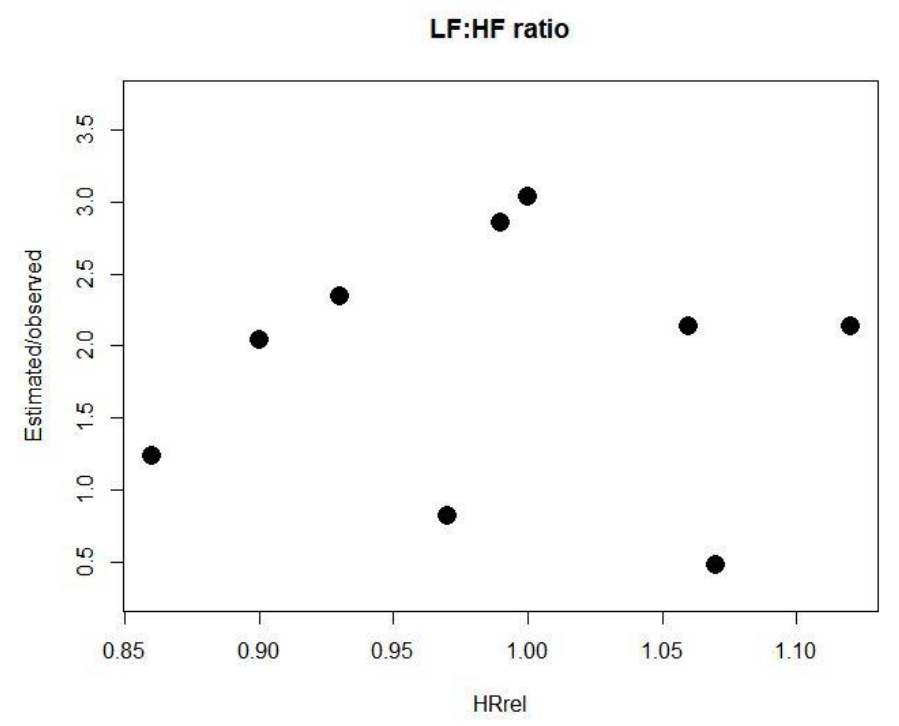

Figure 29 - Ratios between estimated and observed LF:HF ratio power for different combinations of speed and slope.

ratio $=3.48-2.73 \cdot$ speed $-0.08 \cdot$ speed $\cdot$ slope

ratio $=4.04-3.21 \cdot H R_{\text {rel }}$

Table 48. Statistical details for equations 38 and 39.

\begin{tabular}{|c|c|c|c|c|c|c|}
\hline \multicolumn{4}{|c|}{$\mathrm{HR}_{\text {rel }}$} & \multicolumn{3}{|c|}{ Speed and slope } \\
\hline & & error & p-value & & error & p-value \\
\hline intercept & & 0.61 & $<0.001$ & intercept & 0.53 & $<0.001$ \\
\hline \multirow[t]{3}{*}{$\mathrm{HR}_{\mathrm{rel}}$ coef. } & & 0.61 & 0.001 & speed coef. & 0.59 & 0.004 \\
\hline & & & & interaction coef. & 0.03 & 0.06 \\
\hline & $\mathrm{p}$-value & adjusted $r^{2}$ & $\mathrm{~F}$ & $p$-value & adjusted $r^{2}$ & $\mathrm{~F}$ \\
\hline model & 0.001 & 0.77 & 27.63 & 0.004 & 0.78 & 14.78 \\
\hline
\end{tabular}




\begin{tabular}{|c|c|c|}
\hline $\begin{array}{l}\text { Independent } \\
\text { variable }\end{array}$ & DF & RSS \\
\hline$v+v \cdot i$ & 6 & 0.12 \\
\hline \multirow[t]{2}{*}{$\mathrm{HR}_{\text {rel }}$} & 7 & 0.15 \\
\hline & $p$-value & $\mathrm{F}$ \\
\hline Comparison & 0.32 & 1.19 \\
\hline
\end{tabular}

For both populations, from Experiment 2 and 1, the model that best explains $L F: H F$ ratio is the one with $H R_{\text {rel }}$ as the independent variable. In both cases, LF:HF is inversely proportional to $H R_{\text {rel }}$,

\subsubsection{Considerations regarding normalized power and LF:HF ratio}

We find the model fitted for Experiment 2 to be adequate to explain $L_{\mathrm{nu}}$ power band from Experiment 1. On the other hand, the model associated with $\mathrm{HF}_{\mathrm{nu}}$ in Experiment 2 underestimates $\mathrm{HF}_{\mathrm{nu}}$ for Experiment 1.

TULPPO et al. (1998) measured $\mathrm{HF}_{\mathrm{nu}}$ during incremental exercise on a cycle ergometer. Subjects were divided into 3 groups according to age (young - 24 to 34 years, middle-aged - 35 to 46 years and old - 47 to 64 years) and 3 groups according to fitness level measured as peak $\mathrm{VO}_{2}$ (poor - 28 to $37 \mathrm{ml} / \mathrm{kg}$, average - 38 to 45 $\mathrm{ml} / \mathrm{kg}$ and good -46 to $60 \mathrm{ml} / \mathrm{kg}$ ). At low and moderate intensities (up to $150 \mathrm{~W}$ ) subjects with lower fitness level presented lower $\mathrm{HF}_{\mathrm{nu}}$ values when compared to those with higher fitness level. Regarding age, older subjects presented lower $\mathrm{HF}_{\mathrm{nu}}$ only up to $75 \mathrm{~W}$. 
Unlike the aforementioned study, we found a difference in $\mathrm{HF}_{\mathrm{nu}}$ at a high intensity between our two populations. Although our groups cannot be considered an ideal experimental design for isolating the influence of factors such as age and fitness level, those most likely play a role in the results we have obtained.

Some studies have raised the possibility that the amplitude of HF per cent is dependent on factors other than metabolic demand, such as absolute $\mathrm{VO}_{2}$ (PERINI \& VEICSTEINAS, 2003).

In our experiments all subjects seem to reach maximum or near maximum estimated HR, but as our selected speed was adjusted according to HR and older individuals show a lower HR for the same relative exercise intensity, it is possible that older subjects were performing at a higher relative submaximal exercise intensity than those who were younger. This could explain the underestimated values of $\mathrm{HF}_{\mathrm{nu}}$ for at least some integrants of the population that engaged in Experiment 1. Such a hypothesis is supported by reports that an augmented HF power is observed in trained when compared to sedentary subjects due to the higher relative exercise intensity reached by the former (CASADEl et al., 1995).

The underestimation of $\mathrm{HF}_{\mathrm{nu}}$ when comparing Experiment 1 and Experiment 2 results in an overestimation of the ratio LF:HF for Experiment 1. In accordance to our results, LEITCH et al., (2003) reported a lower LF:HF ratio in middle-aged compared to young subjects during treadmill walking at $60 \% \mathrm{HR}_{\max }$.

If we look at what happens with absolute values of those 2 frequency bands, we note that although the model fitted for HF from Experiment 2 is adequate to explain data from Experiment 1, LF is overestimated. More so, a tendency to overestimate $\mathrm{HF}$ power under low $\mathrm{HR}_{\text {rel }}$ and underestimate it at high $\mathrm{HR}_{\text {rel }}$ is observed. A similar pattern is seen for the LF band, although the relationship of the estimated/observed ratio with $\mathrm{HR}_{\text {rel }}$ did not reach significance level. In association with the results presented by sdnn $n_{\text {rel }}$ this points to an overestimation of HRV under the lower intensities adopted and overestimation at the higher intensities, hence HRV changes less throughout the range of HR studied in Experiment 1 than it did in Experiment 2.

These results are more likely associated with differences in age than fitness level between the 2 populations, as subjects were significantly older in Experiment 1 
compared to Experiment 2. Additionally, PERINI \& VEICSTEINAS (2003) reported that no difference was found between spectral power values of professional cyclists and sedentary subjects at the same relative exercise intensity. Similar results were reported regarding long-distance runners and sedentary peers (CARTER et al., 2003)

LUCY et al. (2003), for example, reported no change in HRV power spectrum (both LF and HF bands and total power) when submitting older subjects to a hypoxic environment, despite changes in breathing pattern, blood pressure and heart rate. On the other hand, young subjects submitted to the same protocol showed a marked reduction in $\mathrm{HF}$ and total power. A greater increase in total LF power with upright tilt had also been reported in younger compared to older subjects (JARISCH et al., 1987; LIPSITZ et al., 1990; PAGANI et al., 1986; SIMPSON \& WICKS, 1988 apud LUCY et al., 2003).

\subsubsection{5 $\mathrm{C}$ band $(1.3-1.6 \mathrm{~Hz})$}

In Table 50 we bring absolute and relative $C_{n u}$ band obtained in Experiment 1.

Table 50 - Absolute $\left(s^{2} / \mathrm{Hz}\right)$ and normalized power in $\mathrm{C}_{\mathrm{nu}}$ band in Experiment 2 presented as mean (s.d.).

\begin{tabular}{|c|c|c|c|c|c|c|}
\hline \multirow{3}{*}{$\begin{array}{c}\text { Speed } \\
\text { (fraction } \\
\text { of } V \text { ) }\end{array}$} & \multicolumn{6}{|c|}{ Slope (\%) } \\
\hline & \multicolumn{3}{|c|}{ Absolute $\left(\cdot 10^{-7}\right)$} & \multicolumn{3}{|c|}{ Normalized } \\
\hline & 0 & 2.5 & 4 & 0 & 2.5 & 4 \\
\hline \multirow[t]{2}{*}{0.8} & $0(0)$ & 0.62 & 1.28 & $0(0)$ & 0.004 & 0.011 \\
\hline & & $(2.14)$ & (3.52) & & $(0.014)$ & $(0.032)$ \\
\hline \multirow[t]{2}{*}{0.9} & 0.52 & 0.86 & 2.55 & 0.005 & 0.010 & 0.027 \\
\hline & $(1.81)$ & (1.55) & (2.99) & $(0.018)$ & $(0.022)$ & $(0.041)$ \\
\hline \multirow[t]{2}{*}{1} & 0.94 & 3.46 & 5.12 & 0.016 & 0.047 & 0.073 \\
\hline & $(2.15)$ & (3.97) & (3.96) & $(0.048)$ & $(0.070)$ & $(0.054)$ \\
\hline
\end{tabular}


The comparison between experimental and predicted values indicates that the model used to predict $C_{n u}$ in Experiment 2 is not adequate for data from Experiment 1 $(\mathrm{t}=-3.26 \mathrm{DF}=8, \mathrm{p}$-value $=0.01)$. The ratios between estimated and observed values are shown in Fig. 30. As can be seen, one point $\left(0.85 \mathrm{HR}_{\mathrm{rel}}\right)$ is missing, as it resulted in zero/zero.

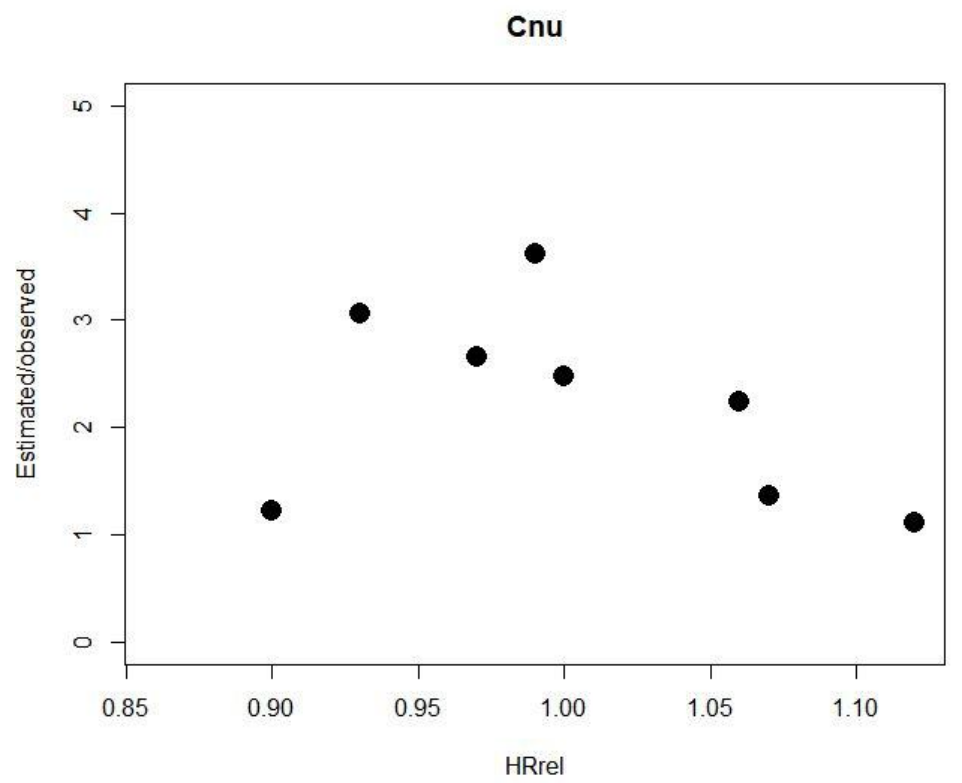

Figure 30 - Ratios between estimated and observed $C_{n u}$ power for different combinations of speed and slope.

We find that the model overestimates $C_{n u}$ for the population in Experiment 1. A linear model of the ratios estimated/observed $C_{\text {nu }}$ per $\mathrm{HR}_{\text {rel }}$ was fitted. The results show that the amount error between predicted and observed values is not dependent on $\mathrm{HR}_{\text {rel }}\left(F=0.98\right.$, adjusted $\mathrm{r}^{2}=-0.003$, p-value $\left.=0.36\right)$.

A multivariable model with speed and slope as the independent variables (equation 40) and a linear regression with $\mathrm{HR}_{\text {rel }}$ as the independent variable (equation 41) were fitted for $C_{n u}$ data obtained from Experiment 1. Statistics of the models and results of their comparison are shown in Tables 51 and 52. Based on such results, we conclude the model of $C_{n u}$ as a function of $H_{\text {rel }}$ is the one that best explains our data.

$$
\begin{aligned}
& C_{n u}=-0.16+0.18 \cdot \text { speed }+0.008 \cdot \text { speed } \cdot \text { slope } \\
& C_{n u}=-0.23+0.26 \cdot H R_{\text {rel }}
\end{aligned}
$$


Table 51 - Statistical details for equations $\mathbf{4 0}$ and 41.

\begin{tabular}{|c|c|c|c|c|c|c|}
\hline \multicolumn{4}{|c|}{$\mathrm{HR}_{\text {rel }}$} & \multicolumn{3}{|c|}{ Speed and slope } \\
\hline & & error & $\mathrm{p}$-value & & error & $p$-value \\
\hline intercept & & 0.05 & 0.002 & intercept & 0.04 & 0.009 \\
\hline \multirow[t]{4}{*}{$\mathrm{HR}_{\text {rel }}$ coef. } & & 0.05 & 0.001 & speed coef. & 0.05 & 0.008 \\
\hline & & & & interaction coef. & 0.002 & 0.02 \\
\hline & $p$-value & adjusted & $\mathrm{F}$ & $\mathrm{p}$-value & adjusted & $\mathrm{F}$ \\
\hline & & $r^{2}$ & & & $r^{2}$ & \\
\hline model & 0.001 & 0.78 & 28.82 & 0.004 & 0.78 & 15.23 \\
\hline
\end{tabular}

Table 52 - Statistical details for the comparison between models.

\begin{tabular}{lrr}
\hline $\begin{array}{l}\text { Independent } \\
\text { variable }\end{array}$ & DF & RSS \\
\hline $\mathrm{v}+\mathrm{v} \cdot \mathrm{i}$ & 6 & 0.0008 \\
$\mathrm{HR} \mathrm{r}_{\mathrm{rel}}$ & 7 & 0.0009 \\
& $\mathrm{p}$-value & $\mathrm{F}$ \\
\hline Comparison & 0.33 & 1.12 \\
\hline $\begin{array}{l}\text { DF degrees of freedom; RSS } \\
\text { sum of squares. }\end{array}$
\end{tabular}

For both populations, from Experiments 2 and 1, the model that best explains $\mathrm{C}_{\mathrm{nu}}$ is the one with $\mathrm{HR}_{\mathrm{rel}}$ as the independent variable. In both cases, $\mathrm{C}_{\mathrm{nu}}$ is directly proportional to $\mathrm{HR}_{\mathrm{rel}}$.

In Question 2 we discuss possible mechanisms associated with $\mathrm{C}_{\mathrm{nu}}$. Briefly, two hypothesis were raised, that of a mechanical mechanism associated with stretching of sinus node due to increased venous return during muscle contraction (BLAIN et al., 2009) and that associated with metaboreceptors in active muscle (FISHER, 2014). Both these alternatives would lead to an increase in sympathetic 
activity. The fact that $\mathrm{C}_{\mathrm{nu}}$ observed in Experiment 1 is overestimated in comparison with Experiment 2 let us believe that results are related to the lower sympathetic responsiveness in this older population (JAKOVLJEVIC, 2017). One could also point out the sympathetic responsiveness is not reduced, but instead, the stimuli that led to its increment are diminished. This is unlikely, as muscle activity must have been similar in both populations, given that it is established by the running conditions.

\subsubsection{In conclusion...}

In Table 53 we present a summary of the results presented above.

Table 53 - Summary the predictions of the data from Experiment 1 based on models from Experiment 2.

\begin{tabular}{|c|c|c|}
\hline Estimator & Is the model adequate? & Observations \\
\hline $\mathrm{HR}_{\text {rel }}$ & no (same tendency) & overestimates \\
\hline sdnn & no (same tendency) & $\begin{array}{l}\text { overestimates for low } \mathrm{HR}_{\mathrm{rel}} \text { and } \\
\text { underestimates for higher } \mathrm{HR}_{\mathrm{rel}}\end{array}$ \\
\hline rms & no (same tendency) & underestimates \\
\hline a1ApEn & yes & - \\
\hline LF (abs) & no (same tendency) & underestimates \\
\hline $\mathrm{LF}_{\mathrm{nu}}$ & yes & - \\
\hline HF (abs) & yes & - \\
\hline $\mathrm{HF}_{\mathrm{nu}}$ & no (same tendency) & underestimates \\
\hline LF:HF ratio & no (same tendency) & overestimates \\
\hline$C_{\text {nu }}$ & no (same tendency) & overestimates \\
\hline
\end{tabular}

Our data indicate that, as hypothesized, the general pattern observed for Experiment 2 is seen in Experiment 1 regarding the response of HRV to the different 
runs. However, in general, the models fitted for the more restricted group that engaged in Experiment 2 do not apply to the broader group that engaged in Experiment 1, even though all subjects were male and relatively fit amateur runners.

The differences in HRV in those that engaged in Experiment 2 compared to Experiment 1 might reflect the possibility that the exercise represented a higher relative intensity to subjects in Experiment 1 than it did to those that engaged in Experiment 2 and the reduced responsiveness of the HR to sympathetic stimuli. As volunteers approach maximum exercise intensity, the decrease in HRV might also approach its minimum values, reducing the estimated error and reducing how much HRV changes in response to the experimental protocol. 


\section{CONCLUSION}

In the present study we addressed how heart rate and heart rate variability respond to changes in speed and slope and whether the patterns observed for a more homogeneous group were applicable to a broader, older population. Our conclusions can be summarized by the following:

- The relationship between speed (fraction of $\mathrm{V}$ ), slope (\%) and $\mathrm{HR}_{\text {rel }}$ is given by the following equation:

$$
H R_{\text {rel }}=0.36+0.643 \cdot \text { speed }+0.033 \cdot \text { speed } \cdot \text { slope }
$$

- HR behaves as predicted by a general equation of cost of locomotion in mammals and avians when it comes to the proportional impact of speed and slope (angle of the gradient), but not regarding the magnitude of such responses; The differences between the predicted and observed pattern in HR must result from alterations in the energy cost of running due to changes in movement pattern and the loss of linearity between HR and metabolic demand at very high exercise intensities;

- The behavior of most HRV estimators could be better explained by changes in relative HR rather than combinations of speed and slope.This indicates that HRV responds primarily to metabolic demand;

- One of our estimators (a1ApEn) could better be explained by the running conditions, that is, speed and slope. We hypothesize that it reflects either a speed or cadence component influencing HRV.

- Although the general pattern of response of HRV to the different running conditions persists when comparing a more homogeneous, younger group to a broader population, how much a given HRV estimators changes in response to the experimental protocol differs between groups, most likely due to age-associated changes in cardiac control. 


\section{RESUMO}

Todo corredor se depara com mudanças de velocidade (v) e inclinação (i) durante o exercício. Correr mais rápido ou em uma superfície mais íngreme representam estímulos diferentes, porém ambos levam a um aumento na demanda metabólica e, portanto, na freqüência cardíaca (FC). O controle da FC é dado por componentes neurais e não neurais e pode ser acessado analisando-se a variabilidade da frequência cardíaca (VFC). Durante o exercício a VFC é influenciada pelos ramos simpático e parassimpático, freqüência ventilatória, cadência, dentre outros componentes. Assim, buscamos abordar como mudanças de velocidade e inclinação durante a corrida impactam a FC e a VFC e se os padrões observados para uma população restrita aplicam-se à um grupo mais amplo. Foram coletados registros de ECG de voluntários divididos em 2 grupos: Experimento 1 ( $N=12$, com faixa etária e nível de condicionamento físico mais amplos) e Experimento $2(\mathrm{~N}=9$, composto por uma população mais homogênea). Os voluntários correram em 9 (Experimento 1) ou 16 (Experimento 2) combinações de velocidade e inclinação por alguns minutos. Nossos dados mostram que a relação entre inclinação (\%), velocidade relativa (v) e $\mathrm{FC}$ relativa é $\mathrm{FC}=0.36+0.643 * \mathrm{v}+0.033^{*} \mathrm{~s} * \mathrm{v}$. A FC se comportou como previsto por uma equação geral do custo de locomoção em mamíferos e aves quando se trata do impacto proporcional de velocidade e inclinação, mas não em relação à magnitude de tais respostas. Isso deve refletir a perda de linearidade entre a demanda metabólica e a FC em intensidades de exercício muito altas. Mudanças na FC explicam o comportamento da maioria dos estimadores de VFC. No entanto, um estimador (a1ApEn) foi melhor explicado por mudanças de velocidade e inclinação. Isso indica que a VFC responde principalmente à FC. O mesmo padrão de resposta da VFC às diferentes condições de corrida foi observado em ambos os grupos, embora, possivelmente devido à mudanças no controle cardíaco associadas à idade, o quanto um determinado estimadores se altera difere entre os grupos. 


\section{ABSTRACT}

Every runner faces changes in speed and slope while exercising. While running faster or on a steepier surface represent different stimuli, both conditions lead to an increase in metabolic demand and therefore in heart rate (HR). The control of HR is given by both neural and non-neural components and can be accessed analyzing the heart rate variability (HRV). During exercise, HRV is known to be influenced by both sympathetic and parasympathetic branches of the autonomic nervous system, by breathing frequency, cadence, amongst other components. Hence, the present study sought to address how changes in speed and slope during running impact HR and HRV and whether the patterns observed for a restricted population were applicable to a broader group. We collected ECG records from volunteers divided into 2 groups: Experiment 1 ( $N=12$, with a broader range of ages and fitness level) and Experiment 2 ( $N=9$, composed of a more homogeneous population). Subjects ran on 9 (Experiment 1) or 16 (Experiment 2) combinations of speed and slope for a few minutes. Our data shows that the relationship between slope (\%), relative speed $(v)$ and relative $\mathrm{HR}$ is $\mathrm{HR}=0.36+0.643{ }^{*} \mathrm{v}+0.033^{*} \mathrm{~s}^{*} \mathrm{v}$. HR behaved as predicted by a general equation of cost of locomotion in mammals and avians when it comes to the proportional impact of speed and slope, but not regarding the magnitude of such responses. This must reflect the loss of linearity between metabolic demand and HR at very high exercise intensities. Changes in HR could explain the behavior of most HRV estimators. However, one estimator (a1ApEn) could better be explained by changes in speed and slope. This indicates that HRV responds primarily to metabolic demand. The same general pattern of response of HRV to the different running conditions was observed in both groups, although, likely due to age-associated changes in cardiac control, how much a given HRV estimators changes differ between groups. 


\section{REFERENCES}

Acharya, U. R., Joseph, K. P., Kannathal, N., Lim, C. M., \& Suri, J. S. (2006). Heart rate variability: A review. Medical and Biological Engineering and Computing, 44(12), 1031-1051. https://doi.org/10.1007/s11517-006-0119-0

Alikhani, I., Noponen, K., \& Sepp, T. (2017). Contribution of Body Movements on the Heart Rate Variability During High Intensity Running. In IEEE Eng Med Biol Soc (pp. 3993-3996).

Bailon, R., Garatachea, N., De La Iglesia, I., Casajus, J. A., \& Laguna, P. (2013). Influence of running stride frequency in heart rate variability analysis during treadmill exercise testing. IEEE Transactions on Biomedical Engineering, 60(7), 1796-1805. https://doi.org/10.1109/TBME.2013.2242328

Blain, G., Meste, O., Blain, A., \& Bermon, S. (2009). Time-frequency analysis of heart rate variability reveals cardiolocomotor coupling during dynamic cycling exercise in humans. Am J Physiol Circ Physiol, 296, 1651-1659. https://doi.org/10.1152/ajpheart.00881.2008.

Carter, J. B., Banister, E. W., \& Blaber, A. P. (2003). Effect of endurance exercise on autonomic control of heart rate. Sports Medicine, 33(1), 33-46. https://doi.org/10.2165/00007256-200333010-00003

Casadei, B., Cochrane, S., Johnsoton, J., Conway, J., \& Sleight, P. (1995). Pitfalls in the interpretation of spectral analysis of the heart rate variability during exercise in humans. Acta Physiologica Scandinavica, 153(2), 125-131. https://doi.org/10.1111/j.1748-1716.1995.tb09843.x

Cavagna, G. A., \& Kaneko, M. (1977). Mechanical work and efficiency in level walking and running. Journal of Physiology, 268(3), 467-481. https://doi.org/10.1113/jphysiol.1977.sp011866

Dong, J. G. (2016). The role of heart rate variability in sports physiology (Review). Experimental and Therapeutic Medicine, 11(5), 1531-1536. https://doi.org/10.3892/etm.2016.3104

Dugan, S. A., \& Bhat, K. P. (2005). Biomechanics and analysis of running gait. Physical Medicine and Rehabilitation Clinics of North America, 16(3), 603-621. https://doi.org/10.1016/j.pmr.2005.02.007

Fisher, J. P. (2014). Autonomic control of the heart during exercise in humans: Role of skeletal muscle afferents. Experimental Physiology, 99(2), 300-305. https://doi.org/10.1113/expphysiol.2013.074377

Folkow, B., Gaskell, P., \& Waaler, B. A. (1970). Blood Flow through Limb Muscles during Heavy Rhythmic Exercise. Acta Physiologica Scandinavica, 80(1), 61-72. https://doi.org/10.1111/j.1748-1716.1970.tb04770.x

Garber, C. E., Blissmer, B., Deschenes, M. R., Franklin, B. A., Lamonte, M. J., Lee, I. M., .. Swain, D. P. (2011). Quantity and quality of exercise for developing and maintaining cardiorespiratory, musculoskeletal, and neuromotor fitness in apparently healthy adults: Guidance for prescribing exercise. Medicine and Science in Sports and Exercise, 43(7), 1334-1359. https://doi.org/10.1249/MSS.0b013e318213fefb

Goldberger, A. L., Amaral, L. A. N., Hausdorff, J. M., Ivanov, P. C., Peng, C.-K., \& Stanley, H. E. (2002). Fractal dynamics in physiology: Alterations with disease and aging. Proceedings of the National Academy of Sciences, 99(Supplement 1), 2466-2472. https://doi.org/10.1073/pnas.012579499

Gregoire, J., Tuck, S., Yamamoto, Y., \& Hughson, R. L. (1996). Heart rate variability at rest and exercise: influence of age, gender, and physical training. Canadian Journal of Applied Physiology = Revue Canadienne de Physiologie Appliquee, 21(6), 455-470. https://doi.org/10.1139/h96-040

Guidelines. (1996). Guidelines Heart rate variability. European Heart Journal, 17, 354-381. https://doi.org/10.1161/01.CIR.93.5.1043 
Hall, J. E. (2011). Guyton and Hall Textbook of Medical Physiology (12e). 2011. https://doi.org/10.1007/s13398-014-0173-7.2

Hanada, E. (2017). Minha vida corredor. Retrieved from http://eduhanada.blogspot.com.br/2017/01/31122016-92-corrida-internacional-de.html

Hermens, H. J., Freriks, B., Merletti, R., Stegeman, D., Blok, J., Rau, G., ... Hägg, G. (1999). European Recommendations for Surface ElectroMyoGraphy Results of the SENIAM project. Roessingh Research and Development, 8(2), 13-54. https://doi.org/10.1016/S1050-6411(00)00027-4

Jakovljevic, D. G. (2017). Physical activity and cardiovascular aging: Physiological and molecular insights. Experimental Gerontology, 109, 67-74. https://doi.org/10.1016/j.exger.2017.05.016

Jones, A. M., \& Doust, J. H. (1996). A 1\% treadmill grade most accurately reflects the energetic cost of outdoor running. Journal of Sports Sciences, 14(4), 321-327. https://doi.org/10.1080/02640419608727717

Kindermann, W., Schnabel, A., Schmitt, W. M., Biro, G., Cassens, J., \& Weber, F. (1982). Catecholamines, Growth Hormone, Cortisol, Insulin, and Sex Hormones in Anaerobic and Aerobic Exercise. European Journal of Applied Physiology and Occupational Physiology, 49(3), 389-399.

Kirby, R. L., Nugent, S. T., Marlow, R. W., MacLeod, D. A., \& Marble, A. E. (1989). Coupling of cardiac and locomotor rhythms. Journal of Applied Physiology (Bethesda, Md.: 1985), 66(1), 323-329. https://doi.org/10.1152/jappl.1989.66.1.323

Leicht, A. S., Allen, G. D., \& Hoey, A. J. (2003). Influence of age and moderate-intensity exercise training on heart rate variability in young and mature adults. Canadian Journal of Applied Physiology = Revue Canadienne de Physiologie Appliquée, 28(3), 446-461. https://doi.org/10.1139/h03-033

Lewis, M. J., \& Short, A. L. (2007). Sample entropy of electrocardiographic RR and QT time-series data during rest and exercise. Physiological Measurement, 28(6), 731-744. https://doi.org/10.1088/0967-3334/28/6/011

Lucy, S. D., Kowalchuk, J. M., Hughson, R. L., Paterson, D. H., \& Cunningham, D. A. (2003). Blunted cardiac autonomic responsiveness to hypoxemic stress in healthy older adults. Can.J Appl Physiol, 28(1066-7814 (Print)), 518-535. https://doi.org/10.1139/h03-040

$\begin{array}{lllll}\text { Maclin, } & \text { G. } & \text { (n.d.). } & \text { Boston } & \text { Marathon. }\end{array}$ http://www.pbase.com/gmaclin/image/96811518/original \%0A

Malik, M., \& Camm, J. (1993). Components of heart rate variability - what they really mean and what we really measure. American Journal of Cardiology, 72, 821-822. https://doi.org/10.3892/or.2017.5549

Mann, R. A., \& Hagy, J. (1980). Biomechanics of walking, running, and sprinting. The American Journal of Sports Medicine, 8(5), 354-350.

McArdle, W. D., Katch, F. I., \& Katch, V. L. (2007). Exercise Physiology: Energy, Nutrition \& Human Performance. (E. Lupash, R. Keifer, R. Kerins, \& J. Montalbano, Eds.) (6th ed.). Philadelphia: Lippincott Williams \& Wilkinks.

Michael, S., Graham, K. S., \& Davis, G. M. (2017). Cardiac Autonomic Responses during Exercise and Post-exercise Recovery Using Heart Rate Variability and Systolic Time Intervals-A Review. Frontiers in Physiology, 8(May), 1-19. https://doi.org/10.3389/fphys.2017.00301

Minetti, A. E., Moia, C., Roi, G. S., Susta, D., \& Ferretti, G. (2002). Energy cost of walking and running at extreme uphill and downhill slopes. Journal of Applied Physiology, 93(3), 1039-1046. https://doi.org/10.1152/japplphysiol.01177.2001 
Natali, J., \& Chaui-Berlinck, J. (2016). Area 1 of Approximate Entropy as a Fast and Robust Tool to Address Temporal Organization. British Journal of Applied Science \& Technology, 13(6), 1-11. https://doi.org/10.9734/BJAST/2016/22726

Natali, J. E. S. (2015). Heart Rate Control in Running. Universidade de São Paulo.

Niizeki, K., Kawahara, K., \& Miyamoto, Y. (1993). Interaction among cardiac, respiratory, and locomotor rhythms during cardiolocomotor synchronization. Journal of Applied Physiology (Bethesda, Md. : 1985), 75(4), 1815-1821. Retrieved from http://eutils.ncbi.nlm.nih.gov/entrez/eutils/elink.fcgi?dbfrom=pubmed\&id=8282636\&retmode=ref\&cmd= prlinks\%5Cnpapers3://publication/uuid/99B466F9-4BE0-48A9-838B-93A634487B54

Novacheck, T. (1998). Review paper: The biomechanics of running. Gait and Posture, 7, 77-95. https://doi.org/10.1016/S0966-6362(97)00038-6

Padulo, J., Powell, D., Milia, R., \& Ardigò, L. P. (2013). A Paradigm of Uphill Running. PLoS ONE, 8(7), 1-8. https://doi.org/10.1371/journal.pone.0069006

Perini, R., Orizio, C., Baselli, G., Cerutti, S., \& Veicsteinas, A. (1990). The influence of exercise intensity on the power spectrum of heart rate variability. European Journal of Applied Physiology and Occupational Physiology, 61(1-2), 143-148. https://doi.org/10.1007/BF00236709

Perini, R., \& Veicsteinas, A. (2003). Heart rate variability and autonomic activity at rest and during exercise in various physiological conditions. European Journal of Applied Physiology, 90(3-4), 317-325. https://doi.org/10.1007/s00421-003-0953-9

Perkiomaki, J. S., Zareba, W., Badilini, F., \& Moss, A. J. (2002). Influence of atropine on fractal and complexity measures of heart rate variability. Annals of Noninvasive Electrocardiology, 7(4), 326-331. https://doi.org/10.1111/j.1542-474X.2002.tb00181.x

Pincus, S. M. (1991). Approximate entropy as a measure of system complexity. Mathematics, 88(March), 2297-2301. https://doi.org/10.1073/pnas.88.6.2297

Santos, B. T., Martins, R. A., Natali, J. E. S., Rodrigues, V. H., Marques, F. S., \& Chauí-Berlinck, J. G. (2009). Consistency in approximate entropy given by a volumetric estimate. Chaos, Solitons and Fractals, 42(1), 322-334. https://doi.org/10.1016/j.chaos.2008.12.002

Sloniger, M. A., Cureton, K. J., Prior, B. M., Evans, E. M., Mark, A., Cureton, K. J. Evans, E. M. (1997). Anaerobic capacity and muscle activation during horizontal and uphill running. Journal of Applied Physiology, 83(1), 262-269.

Stoggl, T., \& Wunsch, T. (2016). Marathon running: Physiology, psychology, nutrition and training aspects. Marathon Running: Physiology, Psychology, Nutrition and Training Aspects. https://doi.org/10.1007/978-3-319-29728-

Sumi, K., Suzuki, S., Matsubara, M., Ando, Y., \& Kobayashi, F. (2006). Heart rate variability during high-intensity field exercise in female distance runners. Scandinavian Journal of Medicine and Science in Sports, 16(5), 314-320. https://doi.org/10.1111/j.1600-0838.2005.00492.x

Takano, N. (1995). Phase relation and breathing pattern during locomotor/respiratory coupling in uphill and downhill running. The Japanese Journal of Physiology, 45(1), 47-58. Retrieved from http://www.ncbi.nlm.nih.gov/pubmed/7650857

Tanaka, H., Monahan, K. D., \& Seals, D. R. (2001). Age-predicted maximal heart rate revisited. Journal of the American College of Cardiology. https://doi.org/10.1016/S0735-1097(00)01054-8

Taylor, C. R., Heglund, N. C., \& Maloiy, G. M. O. (1982). Energetics and mechanics of terrestrial locomotion. Journal of Experimental Biology, 97, 1-21. https://doi.org/10.1109/TMM.2004.830805

Tulppo, M. P., Hughson, R. L., Mäkikallio, T. H., Airaksinen, K. E., Seppänen, T., \& Huikuri, H. V. (2001). Effects of exercise and passive head-up tilt on fractal and complexity properties of heart rate 
dynamics. American Journal of Physiology. Heart and Circulatory Physiology, 280, H1081-H1087. https://doi.org/11179050

Tulppo, M. P., Mäkikallio, T. H., Seppänen, T., Laukkanen, R. T., \& Huikuri, H. V. (1998). Vagal modulation of heart rate during exercise: effects of age and physical fitness. American Journal of Physiology - Heart and Circulatory Physiology, 274(2), H424-H429. https://doi.org/10.1097/00005768-199705001-00813

Tulppo, M. P., Makikallio, T. H., Takala, T. E. S., Seppanen, T., \& Huikuri, H. V. (1996). Analysis of Heart Rate Dynamics During Exercise, 244-252.

Vernillo, G., Giandolini, M., Edwards, W. B., Morin, J. B., Samozino, P., Horvais, N., \& Millet, G. Y. (2017). Biomechanics and Physiology of Uphill and Downhill Running. Sports Medicine, 47(4), 615-629. https://doi.org/10.1007/s40279-016-0605-y 